\title{
The Use of Strain-Release for the Diastereoselective Construction of Quaternary Carbon Centers
}

Tobias Pinkert, Mowpriya Das, Malte L. Schrader, and Frank Glorius*

Supporting Information

Organisch-Chemisches Institut, Westfälische Wilhelms-Universität Münster, Corrensstraße 40, 48149 Münster, Germany

glorius@uni-muenster.de 


\section{Content}

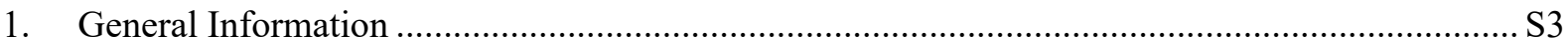

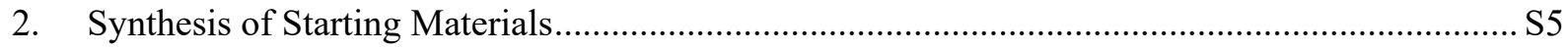

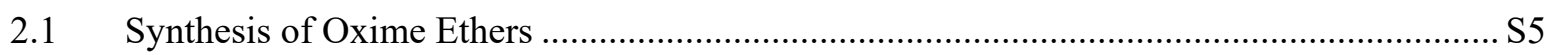

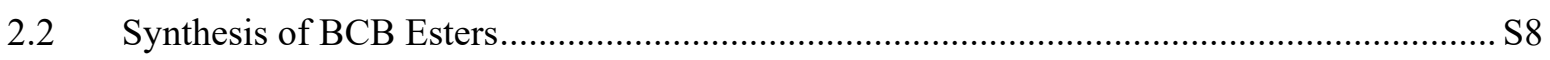

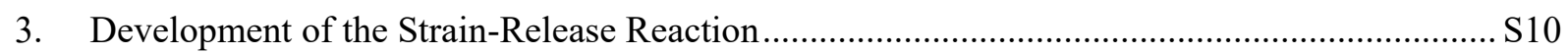

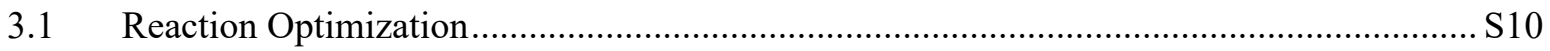

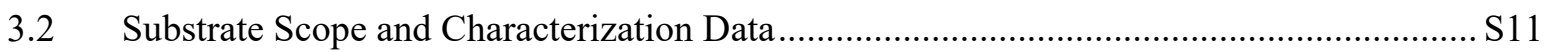

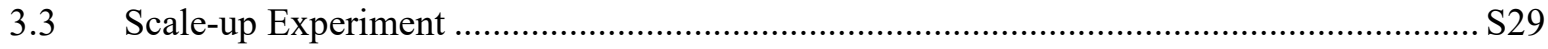

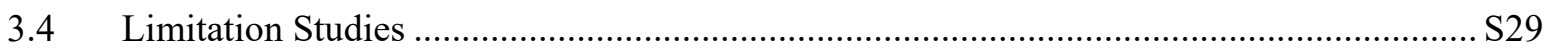

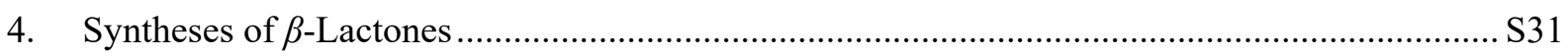

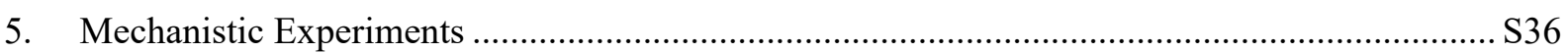

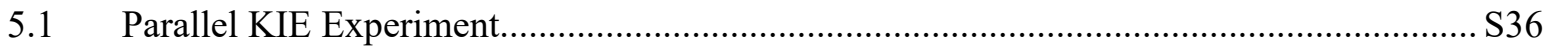

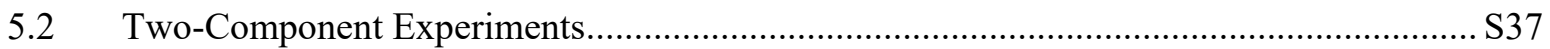

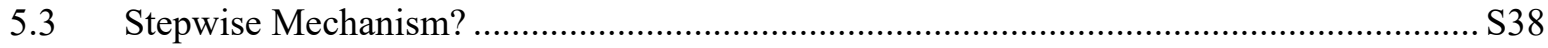

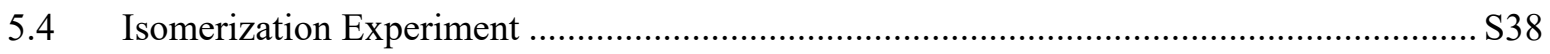

6. Proof of the Stereochemistry by X-Ray Crystallography ............................................................. S39

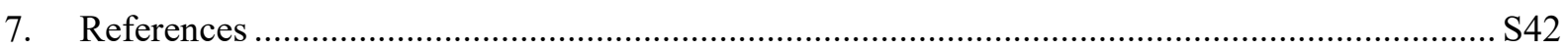

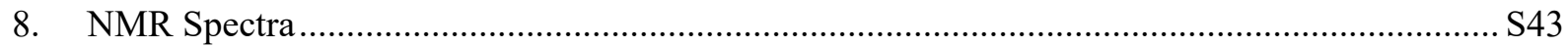




\section{General Information}

Unless otherwise noted, all reactions were carried out in oven-dried Schlenk tubes under an atmosphere of argon. Reaction temperatures are reported as the temperature of the pre-heated heating block or cooling bath, respectively, unless otherwise stated.

Dry solvents $\left(<50 \mathrm{ppm}_{2} \mathrm{O}\right)$ were purchased from Acros Organics, Sigma-Aldrich or Carl Roth and stored over molecular sieves under an argon atmosphere and were transferred under argon. Dichloromethane $\left(\mathrm{CaH}_{2}\right)$, tetrahydrofuran $\left(\mathrm{Na}\right.$ /benzophenone) and toluene $\left(\mathrm{CaH}_{2}\right)$ were kept under an argon atmosphere and freshly distilled before use.

Commercially available chemicals were obtained from ABCR, Acros Organics, Aldrich Chemical Co., Alfa Aesar, Carl Roth, Chempur, Combi Blocks, Fluorochem UK and TCI Europe and used as received unless otherwise stated.

Analytical thin layer chromatography (TLC) was performed on silica gel $60 \mathrm{~F}_{254}$ aluminium plates (Merck). TLC plates were visualized by exposure to short wave ultraviolet light $(254 \mathrm{~nm}, 366 \mathrm{~nm})$ or chemically by potassium permanganate solution and heating subsequently.

Flash column chromatography was performed on silica gel $(35-70 \mu \mathrm{m})$ obtained from Acros Organics by standard techniques. Solvents used for flash column chromatography were distilled before use.

${ }^{1} \mathrm{H},{ }^{13} \mathrm{C}$ and ${ }^{19} \mathrm{~F}$ NMR spectra were recorded on a Bruker Avance II300 or Avance II400, AgilentDD2 500 or AgilentDD2 600 in solvents as indicated. Chemical shifts $(\delta)$ for ${ }^{1} \mathrm{H}$ and ${ }^{13} \mathrm{C}$ NMR spectra are given in ppm relative to TMS. The residual solvent signals were used as references for ${ }^{1} \mathrm{H}$ and ${ }^{13} \mathrm{C}$ spectra and the chemical shifts were converted to the TMS scale $\left(\mathrm{CDCl}_{3}: \delta_{\mathrm{H}}=7.26 \mathrm{ppm}, \delta_{\mathrm{C}}=77.16 \mathrm{ppm}\right) .{ }^{19} \mathrm{~F}$ NMR spectra referenced according to the proton resonance of TMS as the primary reference for the unified chemical shift scale (IUPAC recommendation 2001).

NMR data was analyzed using MestReNova software from Mestrelab Research S.L. Coupling constants $(J)$ are quoted in Hertz. To describe the multiplicities of the signals, the following abbreviations were used: s (singlet), bs (broad singlet), d (doublet), t (triplet), q (quartet), p (quintet), s (sextet), h (heptet), m (multiplet) or combinations.

Exact ESI mass spectra were recorded on a Bruker Daltonics MicroTof. High resolution ESI mass spectra were recorded on a Thermo-Fischer Scientific Orbitrap LTQ XL. Mass Calibration was carried out directly before the measurement of the sample using clusters of sodium formate.

GC-MS spectra were acquired on an Agilent Technology device consisting of an Agilent 7890A, a 5975 inert GC-MSD Mass Selective Detector and an HP-5MS column $(0.25 \mathrm{~mm}$ x $30 \mathrm{~m}, 0.25 \mu \mathrm{m}$ film thickness). The standard method used was 50-40-4.0 (start temperature: $50{ }^{\circ} \mathrm{C}$, temperature gradient: 40 ${ }^{\circ} \mathrm{C} / \mathrm{min}$, end temperature: $290{ }^{\circ} \mathrm{C}$, solvent delay: $4.0 \mathrm{~min}$ ).

LC-UV analysis was performed on a Waters ACQUITY H-Class system with a Waters ACQUITYTUV detector. The liquid chromatography was conducted on an ACQUITY UPLC BEH C18 column, $1.7 \mu \mathrm{m}$ particle size, $2.1 \mathrm{~mm} \times 50 \mathrm{~mm}$, using a gradient of water and acetonitrile.

Reaction optimization studies were analyzed using LC-UV $(\lambda=260 \mathrm{~nm})$ due to a higher absorption of the internal standard 1-fluoronaphthalene. Diastereomeric ratios and site selectivities were determined by LC-UV $(\lambda=254 \mathrm{~nm})$.

Unless otherwise stated the $E / Z$ ratio refers to the configuration of the olefin double bond.

Data sets for compound S3 were collected with a Bruker D8 Venture PHOTON III diffractometer. Programs used: data collection: APEX3 V2019.1-0; ${ }^{1}$ cell refinement: SAINT V8.40A $;{ }^{1}$ data reduction: SAINT V8.40A; ${ }^{1}$ absorption correction, SADABS V2016/2; ${ }^{1}$ structure solution SHELXT-2015; ${ }^{2}$ 
structure refinement $S H E L X L-2015^{3}$ and graphics, $X P .{ }^{4} R$-values are given for observed reflections, and $w \mathrm{R}^{2}$ values are given for all reflections. 


\section{Synthesis of Starting Materials}

\subsection{Synthesis of Oxime Ethers}

(E)-4-(1-(Methoxyimino)ethyl)phenyl trifluoromethanesulfonate (1e)

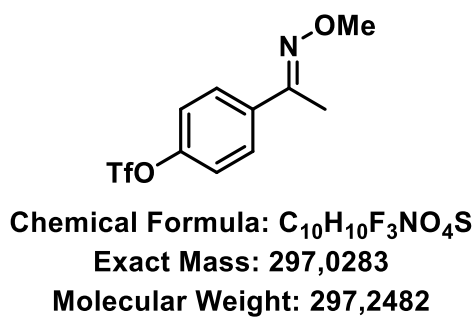

To a solution of $(E)$-1-(4-hydroxyphenyl)ethan-1-one $O$-methyl oxime ( $0.67 \mathrm{~g}, 4.0 \mathrm{mmol}, 1.0$ equiv) and pyridine $(0.65 \mathrm{~mL}, 8.0 \mathrm{mmol}, 2.0$ equiv) in DCM $(8 \mathrm{~mL}, 0.5 \mathrm{M})$ was added triflic anhydride $\left(0.81 \mathrm{~mL}, 4.8 \mathrm{mmol}, 1.2\right.$ equiv) dropwise at $0{ }^{\circ} \mathrm{C}$. The mixture was allowed to warm to room temperature and was stirred for $2 \mathrm{~h}$. The reaction was quenched with $\mathrm{HCl}(1 \mathrm{M})$ and extracted with DCM. The combined organic layers were dried over $\mathrm{Na}_{2} \mathrm{SO}_{4}$ and purification by column chromatography (eluent: pentane $/ \mathrm{Et}_{2} \mathrm{O}$ ) delivered the desired product as a colorless oil.

$\mathbf{R}_{\mathbf{f}}\left(\right.$ pentane $\left./ \mathrm{Et}_{2} \mathrm{O} 98: 2\right)=0.20$.

${ }^{1} \mathbf{H}$ NMR (400 MHz, $\left.\mathrm{CDCl}_{3}\right): \delta(\mathrm{ppm}) 7.77-7.70(\mathrm{~m}, 2 \mathrm{H}), 7.30-7.24(\mathrm{~m}, 2 \mathrm{H}), 4.00(\mathrm{~s}, 3 \mathrm{H}), 2.22(\mathrm{~s}$, $3 \mathrm{H})$.

${ }^{13}$ C NMR (101 MHz, $\left.\mathrm{CDCl}_{3}\right): \delta$ 153.0, 150.0, 137.1, 128.0, 121.5, 118.9 (q, $\left.J=320.9 \mathrm{~Hz}\right), 62.3,12.6$.

${ }^{19}$ F NMR (377 MHz, $\left.\mathrm{CDCl}_{3}\right): \delta-72.8(3 \mathrm{~F})$.

HRMS (ESI): calculated for $\mathrm{C}_{10} \mathrm{H}_{10} \mathrm{~F}_{3} \mathrm{NO}_{4} \mathrm{SH}[\mathrm{M}+\mathrm{H}]^{+}$: 298.0355; found: 298.0356.

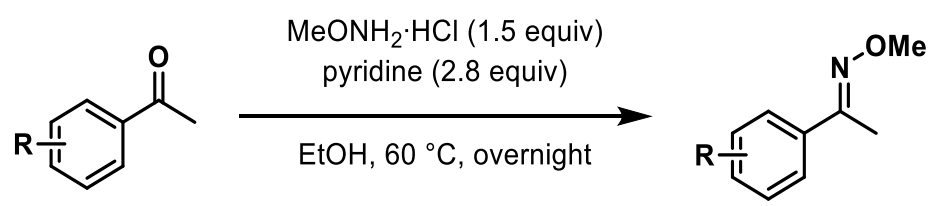

Oxime ethers were synthesized according to a procedure from Chiba: ${ }^{5}$ The respective ketone (1.0 equiv) and pyridine (2.8 equiv) were dissolved in ethanol $(2.2 \mathrm{M})$. Methoxyamine hydrochloride (1.5 equiv) was added and the mixture was stirred overnight at $60{ }^{\circ} \mathrm{C}$. The reaction was quenched by addition of water. The aqueous layer was extracted with EtOAc and the combined organic layers were washed with $\mathrm{HCl}(1 \mathrm{M})$ and dried over $\mathrm{Na}_{2} \mathrm{SO}_{4}$. Purification by column chromatography (eluent: pentane $/ \mathrm{Et}_{2} \mathrm{O}$ or pentane/EtOAc) yielded the desired product.

tert-Butyl (E)-(4-(1-(methoxyimino)ethyl)phenyl)carbamate (1j)

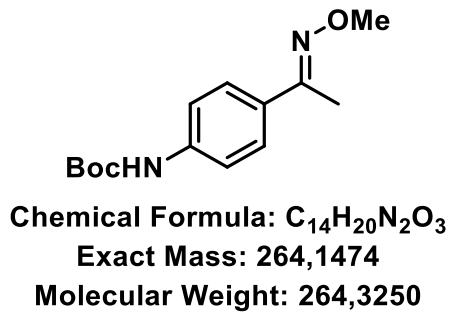


Following the general procedure using tert-butyl (4-acetylphenyl)carbamate $(0.94 \mathrm{~g}, 4.0 \mathrm{mmol}$, 1.0 equiv), methoxyamine hydrochloride $(0.50 \mathrm{~g}, 6.0 \mathrm{mmol}, 1.5$ equiv) and pyridine $(0.9 \mathrm{~mL}$, $11.2 \mathrm{mmol}, 2.8$ equiv) in ethanol $(2.0 \mathrm{~mL}, 2.2 \mathrm{M})$, the title compound was obtained as a white solid.

$\mathbf{R}_{\mathbf{f}}($ pentane/EtOAc 92:8) $=0.31$.

${ }^{1} \mathbf{H}$ NMR $\left(400 \mathrm{MHz}, \mathrm{CDCl}_{3}\right): \delta(\mathrm{ppm}) 7.61-7.55(\mathrm{~m}, 2 \mathrm{H}), 7.40-7.31(\mathrm{~m}, 2 \mathrm{H}), 6.62(\mathrm{~s}, 1 \mathrm{H}), 3.98(\mathrm{~s}$, $3 \mathrm{H}), 2.19(\mathrm{~s}, 3 \mathrm{H}), 1.51(\mathrm{~s}, 9 \mathrm{H})$.

${ }^{13} \mathrm{C}$ NMR (101 MHz, $\left.\mathrm{CDCl}_{3}\right): \delta(\mathrm{ppm}) 154.3,152.6,139.3,131.2,126.9,118.1,80.8,61.9,28.4,12.6$.

HRMS (ESI): calculated for $\mathrm{C}_{14} \mathrm{H}_{20} \mathrm{~N}_{2} \mathrm{O}_{3} \mathrm{Na}[\mathrm{M}+\mathrm{Na}]^{+}:$287.1366; found: 287.1362.

\section{(E)-1-(4-Cyclohexylphenyl)ethan-1-one $O$-methyl oxime (1k)}<smiles>CON=C(C)c1ccc(C2CCCCC2)cc1</smiles>

Chemical Formula: $\mathrm{C}_{15} \mathrm{H}_{21} \mathrm{NO}$

Exact Mass: 231,1623

Molecular Weight: 231,3390

Following the general procedure using 1-(4-cyclohexylphenyl)ethan-1-one (1.01 g, $5.0 \mathrm{mmol}$, 1.0 equiv), methoxyamine hydrochloride $(0.63 \mathrm{~g}, 7.5 \mathrm{mmol}, 1.5$ equiv) and pyridine $(1.14 \mathrm{~mL}$, $14.1 \mathrm{mmol}, 2.8$ equiv) in ethanol $(2.3 \mathrm{~mL}, 2.2 \mathrm{M})$, the title compound was obtained as a white solid.

$\mathbf{R}_{\mathbf{f}}\left(\right.$ pentane $\left./ \mathrm{Et}_{2} \mathrm{O} 98: 2\right)=0.38$.

${ }^{1} \mathbf{H}$ NMR (400 MHz, $\left.\mathrm{CDCl}_{3}\right): \delta(\mathrm{ppm}) 7.57(\mathrm{~d}, J=8.4 \mathrm{~Hz}, 2 \mathrm{H}), 7.21(\mathrm{~d}, J=8.3 \mathrm{~Hz}, 2 \mathrm{H}), 3.99(\mathrm{~s}, 3 \mathrm{H})$, $2.59-2.45(\mathrm{~m}, 1 \mathrm{H}), 2.22(\mathrm{~s}, 3 \mathrm{H}), 1.92-1.81(\mathrm{~m}, 4 \mathrm{H}), 1.79-1.73(\mathrm{~m}, 1 \mathrm{H}), 1.49-1.33(\mathrm{~m}, 4 \mathrm{H}), 1.32-$ $1.21(\mathrm{~m}, 1 \mathrm{H})$.

${ }^{13} \mathrm{C}$ NMR (101 MHz, $\left.\mathrm{CDCl}_{3}\right): \delta$ (ppm) 154.8, 149.3, 134.3, 127.0, 126.1, 62.0, 44.5, 34.5, 27.0, 26.3, 12.8 .

HRMS (ESI): calculated for $\mathrm{C}_{15} \mathrm{H}_{21} \mathrm{NONa}[\mathrm{M}+\mathrm{Na}]^{+}:$254.1515; found: 254.1514 .

\section{(E)-1-(4-(Trifluoromethoxy)phenyl)ethan-1-one $O$-methyl oxime (1r)}

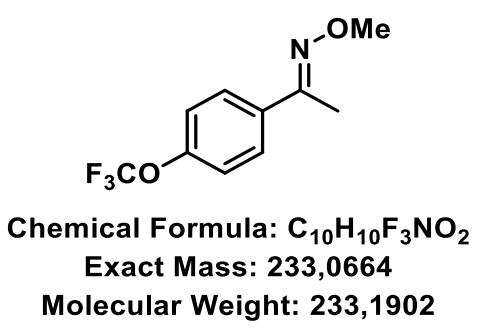

Following the general procedure using 1-(4-(trifluoromethoxy)phenyl)ethan-1-one ( $0.80 \mathrm{~mL}, 5.0 \mathrm{mmol}$, 1.0 equiv), methoxyamine hydrochloride $(0.63 \mathrm{~g}, 7.5 \mathrm{mmol}, 1.5$ equiv) and pyridine $(1.14 \mathrm{~mL}$, $14.1 \mathrm{mmol}, 2.8$ equiv) in ethanol $(2.3 \mathrm{~mL}, 2.2 \mathrm{M})$, the title compound was obtained as a colorless oil.

$\mathbf{R}_{\mathbf{f}}\left(\right.$ pentane $\left./ \mathrm{Et}_{2} \mathrm{O} 98: 2\right)=0.37$. 
${ }^{1} \mathbf{H}$ NMR $\left(400 \mathrm{MHz}, \mathrm{CDCl}_{3}\right): \delta(\mathrm{ppm}) 7.70-7.65(\mathrm{~m}, 2 \mathrm{H}), 7.23-7.19(\mathrm{~m}, 2 \mathrm{H}), 4.00(\mathrm{~s}, 3 \mathrm{H}), 2.22(\mathrm{~s}$, $3 \mathrm{H})$.

${ }^{13}$ C NMR (101 MHz, $\left.\mathrm{CDCl}_{3}\right): \delta(\mathrm{ppm})$ 153.5, $149.9(\mathrm{~m}), 135.4,127.6,120.9(\mathrm{~m}), 120.6(\mathrm{~m}), 62.2,12.7$.

${ }^{19}$ F NMR (377 MHz, $\left.\mathrm{CDCl}_{3}\right): \delta(\mathrm{ppm})-57.8(3 \mathrm{~F})$.

HRMS (ESI): calculated for $\mathrm{C}_{10} \mathrm{H}_{10} \mathrm{~F}_{3} \mathrm{NO}_{2} \mathrm{H}[\mathrm{M}+\mathrm{H}]^{+}$: 234.0736; found: 234.0734.

\section{(E)-4-(1-(Methoxyimino)ethyl)- $N$-phenylbenzenesulfonamide (1t)}

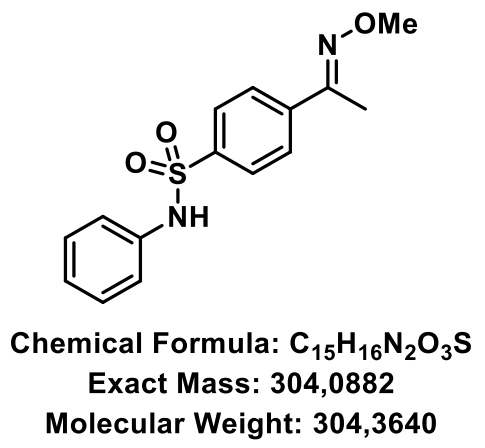

Following the general procedure using 4-acetyl- $N$-phenylbenzenesulfonamide $(1.01 \mathrm{~g}, 3.7 \mathrm{mmol}$, 1.0 equiv), methoxyamine hydrochloride $(0.46 \mathrm{~g}, 5.5 \mathrm{mmol}, 1.5$ equiv) and pyridine $(0.83 \mathrm{~mL}$, $10.3 \mathrm{mmol}, 2.8$ equiv) in ethanol $(1.7 \mathrm{~mL}, 2.2 \mathrm{M})$, the title compound was obtained as a white solid.

$\mathbf{R}_{\mathbf{f}}($ pentane/EtOAc 80:20) $=0.30$.

${ }^{1} \mathbf{H}$ NMR (400 MHz, $\left.\mathrm{CDCl}_{3}\right): \delta(\mathrm{ppm}) 7.78(\mathrm{~d}, J=8.7 \mathrm{~Hz}, 2 \mathrm{H}), 7.70(\mathrm{~d}, J=8.6 \mathrm{~Hz}, 2 \mathrm{H}), 7.25-7.20$ (m, $3 \mathrm{H}), 7.13-7.08(\mathrm{~m}, 3 \mathrm{H}), 4.00(\mathrm{~s}, 3 \mathrm{H}), 2.18(\mathrm{~s}, 3 \mathrm{H})$.

${ }^{13}$ C NMR (101 MHz, $\left.\mathrm{CDCl}_{3}\right): \delta$ (ppm) 153.1, 141.1, 139.1, 136.4, 129.5, 127.5, 126.6, 125.6, 121.7, $62.4,12.5$.

HRMS (ESI): calculated for $\mathrm{C}_{15} \mathrm{H}_{16} \mathrm{~N}_{2} \mathrm{O}_{3} \mathrm{Na}[\mathrm{M}+\mathrm{Na}]^{+}:$327.0774; found: 327.0770 .

\section{(E)-1-(4-(Methylthio)phenyl)ethan-1-one $O$-methyl oxime (1u)}

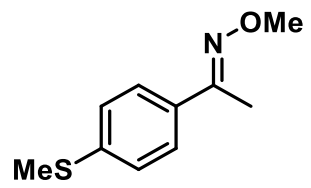

Chemical Formula: $\mathrm{C}_{10} \mathrm{H}_{13} \mathrm{NOS}$

Exact Mass: 195,0718

Molecular Weight: 195,2800

Following the general procedure using 1-(4-(methylthio)phenyl)ethan-1-one $(0.83 \mathrm{~g}, 5.0 \mathrm{mmol}$, 1.0 equiv), methoxyamine hydrochloride $(0.63 \mathrm{~g}, 7.5 \mathrm{mmol}, 1.5$ equiv) and pyridine $(1.14 \mathrm{~mL}$, $14.1 \mathrm{mmol}, 2.8$ equiv) in ethanol $(2.3 \mathrm{~mL}, 2.2 \mathrm{M})$, the title compound was obtained as a white solid.

$\mathbf{R}_{\mathbf{f}}($ pentane/EtOAc 97:3) $=0.36$.

${ }^{1}$ H NMR (400 MHz, $\left.\mathrm{CDCl}_{3}\right): \delta(\mathrm{ppm}) 7.61-7.54(\mathrm{~m}, 2 \mathrm{H}), 7.27-7.19(\mathrm{~m}, 2 \mathrm{H}), 3.99(\mathrm{~s}, 3 \mathrm{H}), 2.49$ (s, $3 \mathrm{H}), 2.20(\mathrm{~s}, 3 \mathrm{H})$.

${ }^{13}$ C NMR (101 MHz, $\left.\mathrm{CDCl}_{3}\right): \delta(\mathrm{ppm}) 154.2,139.9,133.4,126.5,126.2,62.0,15.7,12.5$. 
HRMS (ESI): calculated for $\mathrm{C}_{10} \mathrm{H}_{13} \mathrm{NOSH}[\mathrm{M}+\mathrm{H}]^{+}:$196.0790; found: 196.0790.

\subsection{Synthesis of BCB Esters}

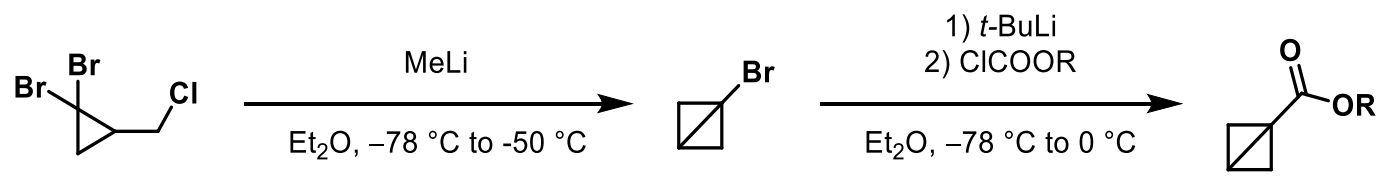

$\mathrm{BCB}$ esters were prepared based on a procedure from Aggarwal: ${ }^{6}$ 1,1-Dibromo-2-(chloromethyl)cyclopropane $\left(2.48 \mathrm{~g}, 10 \mathrm{mmol}, 1.0\right.$ equiv) was dissolved in $\mathrm{Et}_{2} \mathrm{O}(50 \mathrm{~mL}, 0.2 \mathrm{M})$ and cooled to $-78^{\circ} \mathrm{C}$. Methyl lithium $\left(6.3 \mathrm{~mL}\right.$ of a $1.6 \mathrm{M}$ solution in $\mathrm{Et}_{2} \mathrm{O}, 10 \mathrm{mmol}, 1.0$ equiv) was added dropwise and the reaction was stirred for $30 \mathrm{~min}$ at $-78{ }^{\circ} \mathrm{C}$ and $1 \mathrm{~h}$ at $-50{ }^{\circ} \mathrm{C}$. The solution was then cooled back to $-78{ }^{\circ} \mathrm{C}$ and high vacuum was applied for $3 \mathrm{~min}$ to remove volatile bromomethane. $t$ - $\mathrm{BuLi}(5.9 \mathrm{~mL}$ of a $1.7 \mathrm{M}$ solution in pentane, $10 \mathrm{mmol}, 1.0$ equiv) was added dropwise and the reaction was allowed to stir for 20 min at $-78^{\circ} \mathrm{C}$. The mixture was then transferred to a flask containing a solution of the respective chloroformate $\left(15 \mathrm{mmol}, 1.5\right.$ equiv) in $\mathrm{Et}_{2} \mathrm{O}(10 \mathrm{~mL})$ via cannula and stirred for $30 \mathrm{~min}$ at $-78{ }^{\circ} \mathrm{C}$ and then $1 \mathrm{~h}$ at $0{ }^{\circ} \mathrm{C}$. The reaction was quenched with sat. aq. $\mathrm{NH}_{4} \mathrm{Cl}$. The organic layer was separated and the aqueous layer was extracted with $\mathrm{Et}_{2} \mathrm{O}$. The combined organic layers were dried over $\mathrm{Na}_{2} \mathrm{SO}_{4}$ and purification by column chromatography (eluent: pentane $/ \mathrm{Et}_{2} \mathrm{O}$ ) yielded the desired $\mathrm{BCB}$ ester.

\section{Benzyl bicyclo[1.1.0]butane-1-carboxylate (2a)}

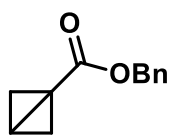

Chemical Formula: $\mathrm{C}_{12} \mathrm{H}_{12} \mathrm{O}_{2}$

Exact Mass: 188,0837

Molecular Weight: 188,2260

Following the general procedure using benzyl chloroformate $(2.14 \mathrm{~mL}, 15 \mathrm{mmol}, 1.5$ equiv), the title compound was obtained as a colorless oil.

$\mathbf{R}_{\mathbf{f}}\left(\right.$ pentane $\left./ \mathrm{Et}_{2} \mathrm{O} 95: 5\right)=0.16$.

${ }^{1}$ H NMR (400 MHz, $\left.\mathrm{CDCl}_{3}\right): \delta(\mathrm{ppm}) 7.39-7.29(\mathrm{~m}, 5 \mathrm{H}), 5.15(\mathrm{~s}, 2 \mathrm{H}), 2.39$ (dt, $\left.J=3.5,1.1 \mathrm{~Hz}, 2 \mathrm{H}\right)$, 2.10 (p, $J=3.2 \mathrm{~Hz}, 1 \mathrm{H}), 1.17(\mathrm{dt}, J=3.0,1.1 \mathrm{~Hz}, 2 \mathrm{H})$.

${ }^{13}$ C NMR (101 MHz, $\left.\mathrm{CDCl}_{3}\right): \delta$ (ppm) 173.1, 136.4, 128.7, 128.3, 128.1, 66.5, 35.8, 17.0, 9.3.

GC-MS: 147.0 (18), 143.0 (10), 129.0 (15), 92.0 (14), 91.0 (100), 81.0 (15), 77.0 (14), 65.0 (22), 53.0 (25), $51.0(14)$. 


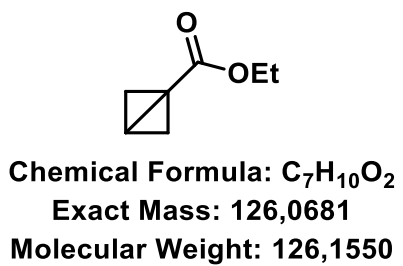

Following the general procedure using ethyl chloroformate $(1.43 \mathrm{~mL}, 15 \mathrm{mmol}, 1.5$ equiv), the title compound was obtained as a colorless oil.

$\mathbf{R}_{\mathbf{f}}\left(\right.$ pentane $\left./ \mathrm{Et}_{2} \mathrm{O} 95: 5\right)=0.19$.

${ }^{1}$ H NMR $\left(400 \mathrm{MHz}, \mathrm{CDCl}_{3}\right): \delta(\mathrm{ppm}) 4.15(\mathrm{q}, J=7.1 \mathrm{~Hz}, 2 \mathrm{H}), 2.35(\mathrm{dt}, J=3.5,1.0 \mathrm{~Hz}, 2 \mathrm{H}), 2.06$ (p, $J=3.2 \mathrm{~Hz}, 1 \mathrm{H}), 1.25(\mathrm{t}, J=7.1 \mathrm{~Hz}, 3 \mathrm{H}), 1.14(\mathrm{dt}, J=2.9,1.0 \mathrm{~Hz}, 2 \mathrm{H})$.

${ }^{13}$ C NMR (101 MHz, $\left.\mathrm{CDCl}_{3}\right): \delta(\mathrm{ppm})$ 173.3, 60.8, 35.6, 16.5, 14.4, 9.2.

GC-MS: 126.1 ([M] $\left.{ }^{+}, 12\right), 98.0$ (71), 81.1 (30), 70.1 (10), 69.1 (26), 54.1 (11), 53.1 (100), 52.1 (19), $51.1(25), 50.1$ (17), $42.1(11), 39.1(12)$.

Isobutyl bicyclo[1.1.0]butane-1-carboxylate (2c)

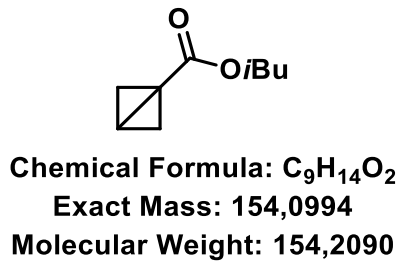

Following the general procedure using isobutyl chloroformate (1.95 mL, $15 \mathrm{mmol}, 1.5$ equiv), the title compound was obtained as a pale yellow oil.

$\mathbf{R}_{\mathbf{f}}($ pentane/Et $2 \mathrm{O} 95: 5)=0.22$.

${ }^{1} \mathbf{H}$ NMR $\left(400 \mathrm{MHz}, \mathrm{CDCl}_{3}\right): \delta(\mathrm{ppm}) 3.88(\mathrm{~d}, J=6.7 \mathrm{~Hz}, 2 \mathrm{H}), 2.36(\mathrm{~m}, 2 \mathrm{H}), 2.05(\mathrm{p}, J=3.1 \mathrm{~Hz}, 1 \mathrm{H})$, $2.00-1.85(\mathrm{~m}, 1 \mathrm{H}), 1.19-1.12(\mathrm{~m}, 2 \mathrm{H}), 0.92(\mathrm{~d}, J=6.7 \mathrm{~Hz}, 6 \mathrm{H})$.

${ }^{13}$ C NMR (101 MHz, $\left.\mathrm{CDCl}_{3}\right): \delta(\mathrm{ppm}) 173.3,70.8,35.6,28.0,19.2,16.5,9.2$.

GC-MS: 99.1 (16), 98.1 (67), 81.1 (58), 69.1 (16), 57.1 (27), 56.1 (14), 55.1 (11), 53.1 (100), 52.1 (14), 51.1 (20), 50.1 (13), 43.1 (13), 42.1 (10), 41.1 (54), 39.1 (26).

$N$-methoxy- $N$-methylbicyclo[1.1.0]butane-1-carboxamide $\mathbf{2 d}$ was prepared following a procedure from Gryko. ${ }^{7}$ 


\section{Development of the Strain-Release Reaction}

\subsection{Reaction Optimization}

An oven-dried $10 \mathrm{~mL}$ Schlenk tube equipped with a stir bar was charged with the respective additive in a glovebox. [Cp* Rh $\left.\left(\mathrm{CH}_{3} \mathrm{CN}\right)_{3}\right]\left(\mathrm{SbF}_{6}\right)_{2}(4.2 \mathrm{mg}, 0.005 \mathrm{mmol}, 5 \mathrm{~mol} \%)$, solvent $(2 \mathrm{~mL}, 0.1 \mathrm{M}),(E)-1-$ phenylethan-1-one $O$-methyl oxime $1 \mathrm{a}$ (14.9 mg, $0.1 \mathrm{mmol}, 1.0$ equiv), benzyl bicyclo[1.1.0]butane-1carboxylate $2 \mathrm{a}$ ( $18.8 \mathrm{mg}, 0.1 \mathrm{mmol}, 1.0$ equiv) and ethyl glyoxylate 3 ( $40.5 \mu \mathrm{L}$ of a $50 \mathrm{wt} \%$ solution in toluene, $0.2 \mathrm{mmol}, 2.0$ equiv) were added sequentially under a positive stream of argon. The tube was sealed and the reaction was stirred for $16 \mathrm{~h}$ at the indicated temperature. The reaction was cooled to room temperature and 1-fluoronaphthalene $(13.0 \mu \mathrm{L}, 0.10 \mathrm{mmol})$ was added as an internal standard. An aliquot $(5 \mu \mathrm{L})$ was taken, filtered through a plug of Celite and analyzed by LC-UV.

Table S1. Optimization studies.

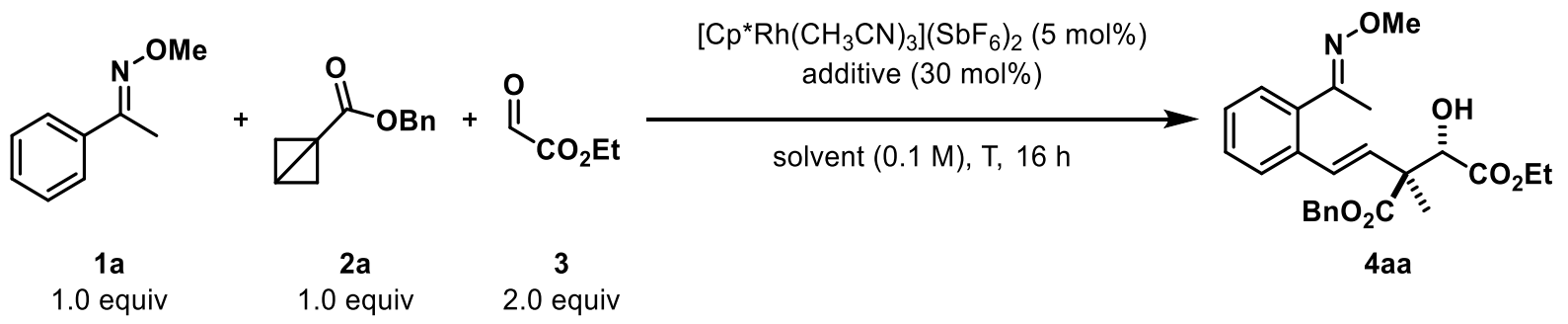

\begin{tabular}{|c|c|c|c|c|c|}
\hline entry & solvent & additive & temperature & $d r$ & yield \\
\hline 1 & TFE & $\mathrm{CsOAc}$ & $60{ }^{\circ} \mathrm{C}$ & $>20: 1$ & $60 \%$ \\
\hline 2 & DCE & $\mathrm{CsOAc}$ & $60{ }^{\circ} \mathrm{C}$ & - & traces \\
\hline 3 & 1,4-dioxane & $\mathrm{CsOAc}$ & $60^{\circ} \mathrm{C}$ & - & - \\
\hline 4 & HFIP & $\mathrm{CsOAc}$ & $60^{\circ} \mathrm{C}$ & $>20: 1$ & $8 \%$ \\
\hline 5 & DMF & $\mathrm{CsOAc}$ & $60^{\circ} \mathrm{C}$ & - & - \\
\hline 6 & TFE & $\mathrm{CsOAc}$ & $\mathrm{rt}$ & $>20: 1$ & $9 \%$ \\
\hline 7 & TFE & $\mathrm{CsOAc}$ & $40^{\circ} \mathrm{C}$ & $>20: 1$ & $40 \%$ \\
\hline 8 & TFE & $\mathrm{CsOAc}$ & $80^{\circ} \mathrm{C}$ & $>20: 1$ & $47 \%$ \\
\hline 9 & TFE & $\mathrm{Na}_{2} \mathrm{CO}_{3}$ & $60^{\circ} \mathrm{C}$ & $>20: 1$ & $28 \%$ \\
\hline 10 & TFE & KOAc & $60^{\circ} \mathrm{C}$ & $>20: 1$ & $55 \%$ \\
\hline 11 & TFE & $\mathrm{NaOAc}$ & $60^{\circ} \mathrm{C}$ & $>20: 1$ & $49 \%$ \\
\hline 12 & TFE & $\mathrm{K}_{3} \mathrm{PO}_{4}$ & $60^{\circ} \mathrm{C}$ & $>20: 1$ & $30 \%$ \\
\hline 13 & TFE & PivOH & $60^{\circ} \mathrm{C}$ & $>20: 1$ & $32 \%$ \\
\hline $14^{[a]}$ & TFE & CsOAc & $60{ }^{\circ} \mathrm{C}$ & $>20: 1$ & $82 \%$ \\
\hline $15^{[\mathrm{b}]}$ & TFE & $\mathrm{CsOAc}$ & $60{ }^{\circ} \mathrm{C}$ & $>20: 1$ & $81 \%$ \\
\hline $16^{[\mathrm{c}]}$ & TFE & $\mathrm{CsOAc}$ & $60^{\circ} \mathrm{C}$ & - & - \\
\hline $17^{[\mathrm{d}]}$ & TFE & CsOAc & $60^{\circ} \mathrm{C}$ & - & - \\
\hline $18^{[\mathrm{e}]}$ & TFE & $\mathrm{CsOAc}$ & $60{ }^{\circ} \mathrm{C}$ & - & - \\
\hline $19^{[\mathrm{f}]}$ & TFE & - & $60^{\circ} \mathrm{C}$ & $>20: 1$ & $52 \%$ \\
\hline
\end{tabular}

Conditions: 1a $(0.10 \mathrm{mmol}), 2 \mathrm{2a}(0.10 \mathrm{mmol}), 3(0.20 \mathrm{mmol}),\left[\mathrm{Cp}^{*} \mathrm{Rh}\left(\mathrm{CH}_{3} \mathrm{CN}\right)_{3}\right]\left(\mathrm{SbF}_{6}\right)_{2}(0.005 \mathrm{mmol}, 5 \mathrm{~mol} \%), \mathrm{CsOAc}$ $\left(0.03 \mathrm{mmol}, 0.3\right.$ equiv). [a] 2.0 equiv of $\mathbf{2 a}$ were used. [b] 2.0 equiv of $\mathbf{1 a}$ were used. [c] [Cp*Co(CO) $\mathrm{I}_{2}$ ] $(2.4 \mathrm{mg}, 0.005 \mathrm{mmol}$,

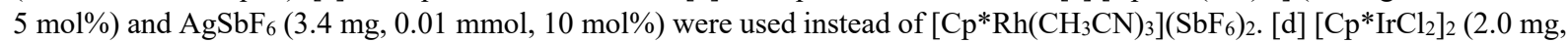
$0.0025 \mathrm{mmol}, 2.5 \mathrm{~mol} \%)$ and $\mathrm{AgSbF}_{6}(3.4 \mathrm{mg}, 0.01 \mathrm{mmol}, 10 \mathrm{~mol} \%)$ were used instead of $\left[\mathrm{Cp}^{*} \mathrm{Rh}\left(\mathrm{CH}_{3} \mathrm{CN}\right)_{3}\right]\left(\mathrm{SbF}_{6}\right)_{2}$. [e] No 
$\left[\mathrm{Cp} * \mathrm{Rh}\left(\mathrm{CH}_{3} \mathrm{CN}\right)_{3}\right]\left(\mathrm{SbF}_{6}\right)_{2}$. [f] No CsOAc. TFE = 2,2,2-trifluoroethanol. HFIP = hexafluoroisopropanol. $\mathrm{rt}=$ room temperature. $\mathrm{PivOH}=$ pivalic acid.

\subsection{Substrate Scope and Characterization Data}

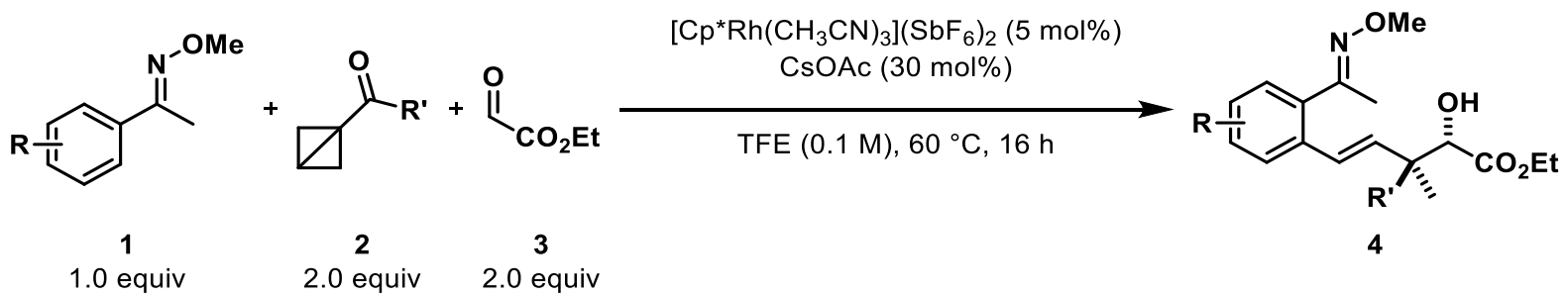

An oven-dried $10 \mathrm{~mL}$ Schlenk tube equipped with a stir bar was charged with CsOAc $(11.5 \mathrm{mg}$, $0.06 \mathrm{mmol}, 0.3$ equiv) in a glovebox. [Cp* $\left.\mathrm{Rh}\left(\mathrm{CH}_{3} \mathrm{CN}\right)_{3}\right]\left(\mathrm{SbF}_{6}\right)_{2}(8.3 \mathrm{mg}, 0.01 \mathrm{mmol}, 5 \mathrm{~mol} \%)$, TFE $(2 \mathrm{~mL}, 0.1 \mathrm{M})$, oxime ether $1(0.20 \mathrm{mmol}, 1.0$ equiv), BCB ester 2 ( $0.40 \mathrm{mmol}, 2.0$ equiv) and ethyl glyoxylate $3(81.5 \mu \mathrm{L}$ of a $50 \mathrm{wt} \%$ solution in toluene, $0.40 \mathrm{mmol}, 2.0$ equiv) were added sequentially under a positive stream of argon. The tube was sealed and the reaction was stirred for $16 \mathrm{~h}$ at $60^{\circ} \mathrm{C}$. The reaction was cooled to room temperature and an aliquot $(5 \mu \mathrm{L})$ was taken, filtered through a plug of Celite and analyzed by LC-UV. The reaction mixture was then concentrated and purified by chromatographic methods to deliver the desired product.

\section{( \pm )-1-Benzyl 4-ethyl $\quad(2 S, 3 S)$-3-hydroxy-2-((E)-2-((E)-1-(methoxyimino)ethyl)styryl)-2-methyl- succinate (4aa)}

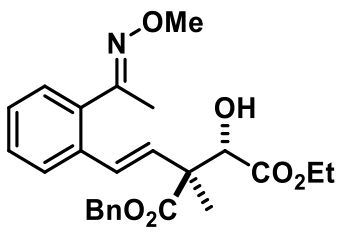

Chemical Formula: $\mathrm{C}_{25} \mathrm{H}_{29} \mathrm{NO}_{6}$

Exact Mass: 439,1995

Molecular Weight: $\mathbf{4 3 9 , 5 0 8 0}$

Prepared from (E)-1-phenylethan-1-one $O$-methyl oxime 1a $(29.8 \mathrm{mg}, 0.20 \mathrm{mmol}, 1.0$ equiv) and benzyl bicyclo[1.1.0]butane-1-carboxylate $\mathbf{2 a}(75.3 \mathrm{mg}, 0.40 \mathrm{mmol}, 2.0$ equiv) according to the general procedure in the presence of $\left[\mathrm{Cp}^{*} \mathrm{Rh}\left(\mathrm{CH}_{3} \mathrm{CN}\right)_{3}\right]\left(\mathrm{SbF}_{6}\right)_{2}(8.3 \mathrm{mg}, 0.01 \mathrm{mmol}, 5 \mathrm{~mol} \%)$. The desired product was obtained after column chromatography (eluent: pentane/EtOAc) and subsequent preparative TLC (eluent: toluene/EtOAc) as a yellowish sticky oil (64.9 mg, $0.15 \mathrm{mmol}, 74 \%,>20: 1 \mathrm{dr}$, $E / Z:>20: 1)$.

$\mathbf{R}_{\mathbf{f}}($ pentane/EtOAc 80:20) $=0.32$.

${ }^{1} \mathbf{H}$ NMR $\left(400 \mathrm{MHz}, \mathrm{CDCl}_{3}\right): \delta(\mathrm{ppm}) 7.46(\mathrm{~d}, J=7.5 \mathrm{~Hz}, 1 \mathrm{H}), 7.37-7.25(\mathrm{~m}, 8 \mathrm{H}), 6.69(\mathrm{~d}, J=16.2$ $\mathrm{Hz}, 1 \mathrm{H}), 6.38(\mathrm{~d}, J=16.2 \mathrm{~Hz}, 1 \mathrm{H}), 5.18(\mathrm{~s}, 2 \mathrm{H}), 4.64(\mathrm{~d}, J=5.5 \mathrm{~Hz}, 1 \mathrm{H}), 4.24-4.08(\mathrm{~m}, 2 \mathrm{H}), 3.96$ (s, $3 \mathrm{H}), 3.18(\mathrm{~d}, J=5.6 \mathrm{~Hz}, 1 \mathrm{H}), 2.11(\mathrm{~s}, 3 \mathrm{H}), 1.42(\mathrm{~s}, 3 \mathrm{H}), 1.20(\mathrm{t}, J=7.1 \mathrm{~Hz}, 3 \mathrm{H})$.

${ }^{13}$ C NMR (101 MHz, $\left.\mathrm{CDCl}_{3}\right): \delta(\mathrm{ppm}) 172.9,172.6,156.5,136.4,135.7,135.4,131.0,129.9,128.8$, $128.6,128.6,128.4,128.2,127.8,126.9,75.0,67.1,62.3,61.9,53.3,16.7,16.7,14.2$.

HRMS (ESI): calculated for $\mathrm{C}_{25} \mathrm{H}_{29} \mathrm{NO}_{6} \mathrm{Na}[\mathrm{M}+\mathrm{Na}]^{+}: 462.1887$; found: 462.1878 . 


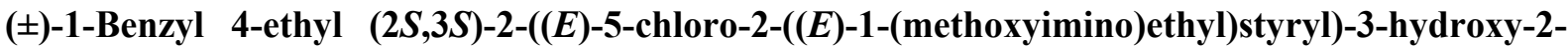
methylsuccinate (4ba)<smiles>CCOC(=O)[C@H](O)C(C)(C)C(=O)OCc1cc(Cl)ccc1/C(C)=N/OC</smiles>

Chemical Formula: $\mathrm{C}_{25} \mathrm{H}_{28} \mathrm{CINO}_{6}$

Exact Mass: 473,1605

Molecular Weight: 473,9500

Prepared from (E)-1-(4-chlorophenyl)ethan-1-one $O$-methyl oxime $1 \mathbf{b}(36.7 \mathrm{mg}, 0.20 \mathrm{mmol}, 1.0$ equiv) and benzyl bicyclo[1.1.0]butane-1-carboxylate 2a (75.3 $\mathrm{mg}, 0.40 \mathrm{mmol}, 2.0$ equiv) according to the general procedure in the presence of $\left[\mathrm{Cp}^{*} \mathrm{Rh}\left(\mathrm{CH}_{3} \mathrm{CN}\right)_{3}\right]\left(\mathrm{SbF}_{6}\right)_{2}(8.3 \mathrm{mg}, 0.01 \mathrm{mmol}, 5 \mathrm{~mol} \%)$. The desired product was obtained after column chromatography (eluent: pentane/EtOAc) and subsequent preparative TLC (eluent: toluene/EtOAc) as a yellowish sticky oil $(65.1 \mathrm{mg}, 0.14 \mathrm{mmol}, 69 \%,>20: 1 \mathrm{dr}$, $E / Z:>20: 1)$.

$\mathbf{R}_{\mathbf{f}}($ pentane $/$ EtOAc 80:20) $=0.35$.

${ }^{1} \mathbf{H}$ NMR $\left(400 \mathrm{MHz}, \mathrm{CDCl}_{3}\right): \delta(\mathrm{ppm}) 7.44(\mathrm{~d}, J=2.0 \mathrm{~Hz}, 1 \mathrm{H}), 7.39-7.30(\mathrm{~m}, 5 \mathrm{H}), 7.25-7.18(\mathrm{~m}$, $2 \mathrm{H}), 6.63(\mathrm{~d}, J=16.3 \mathrm{~Hz}, 1 \mathrm{H}), 6.38(\mathrm{~d}, J=16.2 \mathrm{~Hz}, 1 \mathrm{H}), 5.24-5.13(\mathrm{~m}, 2 \mathrm{H}), 4.64(\mathrm{~d}, J=5.5 \mathrm{~Hz}, 1 \mathrm{H})$, $4.26-4.07(\mathrm{~m}, 2 \mathrm{H}), 3.96(\mathrm{~s}, 3 \mathrm{H}), 3.27(\mathrm{~d}, J=5.5 \mathrm{~Hz}, 1 \mathrm{H}), 2.09$ (s, 3H), $1.41(\mathrm{~s}, 3 \mathrm{H}), 1.21(\mathrm{t}, J=7.1$ $\mathrm{Hz}, 3 \mathrm{H})$.

${ }^{13}$ C NMR (101 MHz, $\left.\mathrm{CDCl}_{3}\right): \delta(\mathrm{ppm}) 172.6,172.6,155.5,137.2,135.6,134.8,134.7,132.3,130.0$, $128.8,128.7,128.4,128.3,127.7,126.8,74.9,67.2,62.4,62.0,53.3,16.6,16.6,14.2$.

HRMS (ESI): calculated for $\mathrm{C}_{25} \mathrm{H}_{28} \mathrm{ClNO}_{6} \mathrm{Na}[\mathrm{M}+\mathrm{Na}]^{+}$: 496.1497; found: 496.1493 .

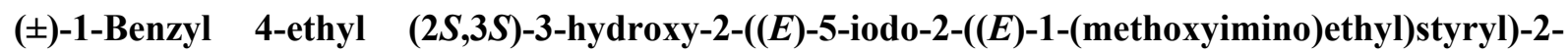
methylsuccinate (4ca)<smiles>CCOC(=O)[C@H](O)[C@@](C)(/C=C/c1cc(I)ccc1/C(C)=N/OC)C(=O)O</smiles>

Chemical Formula: $\mathrm{C}_{25} \mathrm{H}_{28} 1 \mathrm{NO}_{6}$

Exact Mass: 565,0961

Molecular Weight: 565,4045

Prepared from $(E)$-1-(4-iodophenyl)ethan-1-one $O$-methyl oxime 1c $(55.0 \mathrm{mg}, 0.20 \mathrm{mmol}, 1.0$ equiv) and benzyl bicyclo[1.1.0]butane-1-carboxylate 2a (75.3 $\mathrm{mg}, 0.40 \mathrm{mmol}, 2.0$ equiv) according to the general procedure in the presence of $\left[\mathrm{Cp}^{*} \mathrm{Rh}\left(\mathrm{CH}_{3} \mathrm{CN}\right)_{3}\right]\left(\mathrm{SbF}_{6}\right)_{2}(8.3 \mathrm{mg}, 0.01 \mathrm{mmol}, 5 \mathrm{~mol} \%)$. The desired product was obtained after column chromatography (eluent: pentane/EtOAc) and subsequent preparative TLC (eluent: toluene/EtOAc) as a yellowish sticky oil ( $74.2 \mathrm{mg}, 0.13 \mathrm{mmol}, 66 \%,>20: 1 \mathrm{dr}$, $E / Z:>20: 1)$.

$\mathbf{R}_{\mathbf{f}}($ pentane $/$ EtOAc 80:20) $=0.30$.

${ }^{1} \mathbf{H}$ NMR $\left(400 \mathrm{MHz}, \mathrm{CDCl}_{3}\right): \delta(\mathrm{ppm}) 7.79(\mathrm{~d}, J=1.9 \mathrm{~Hz}, 1 \mathrm{H}), 7.58(\mathrm{dd}, J=8.1,1.8 \mathrm{~Hz}, 1 \mathrm{H}), 7.40-$ $7.30(\mathrm{~m}, 5 \mathrm{H}), 6.99$ (d, $J=8.1 \mathrm{~Hz}, 1 \mathrm{H}), 6.58(\mathrm{~d}, J=16.2 \mathrm{~Hz}, 1 \mathrm{H}), 6.36(\mathrm{~d}, J=16.2 \mathrm{~Hz}, 1 \mathrm{H}), 5.22-5.13$ 
$(\mathrm{m}, 2 \mathrm{H}), 4.63(\mathrm{~d}, J=5.4 \mathrm{~Hz}, 1 \mathrm{H}), 4.24-4.08(\mathrm{~m}, 2 \mathrm{H}), 3.95(\mathrm{~s}, 3 \mathrm{H}), 3.26(\mathrm{~d}, J=5.5 \mathrm{~Hz}, 1 \mathrm{H}), 2.08(\mathrm{~s}$, $3 \mathrm{H}), 1.40(\mathrm{~s}, 3 \mathrm{H}), 1.21(\mathrm{t}, J=7.2 \mathrm{~Hz}, 3 \mathrm{H})$.

${ }^{13}$ C NMR (101 MHz, $\left.\mathrm{CDCl}_{3}\right): \delta(\mathrm{ppm}) 172.6,172.5,155.6,137.5,136.6,135.8,135.7,135.6,132.3$, $130.2,128.7,128.6,128.4,128.3,94.8,74.9,67.2,62.4,62.1,53.3,16.6,16.5,14.2$.

HRMS (ESI): calculated for $\mathrm{C}_{25} \mathrm{H}_{28} \mathrm{INO}_{6} \mathrm{Na}[\mathrm{M}+\mathrm{Na}]^{+}: 588.0854$; found: 588.0853 .

( \pm -1-Benzyl 4-ethyl (2S,3S)-2-((E)-5-fluoro-2-((E)-1-(methoxyimino)ethyl)styryl)-3-hydroxy-2methylsuccinate (4da)

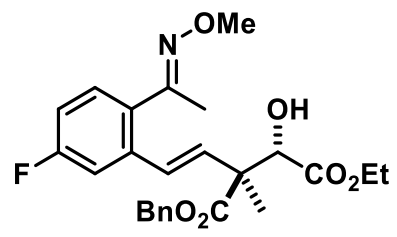

Chemical Formula: $\mathrm{C}_{25} \mathrm{H}_{28} \mathrm{FNO}_{6}$

Exact Mass: 457,1901

Molecular Weight: 457,4984

Prepared from (E)-1-(4-fluorophenyl)ethan-1-one $O$-methyl oxime 1d (33.4 mg, $0.20 \mathrm{mmol}, 1.0$ equiv) and benzyl bicyclo[1.1.0]butane-1-carboxylate $2 \mathrm{a}(75.3 \mathrm{mg}, 0.40 \mathrm{mmol}, 2.0$ equiv) according to the general procedure in the presence of $\left[\mathrm{Cp}^{*} \mathrm{Rh}\left(\mathrm{CH}_{3} \mathrm{CN}\right)_{3}\right]\left(\mathrm{SbF}_{6}\right)_{2}(8.3 \mathrm{mg}, 0.01 \mathrm{mmol}, 5 \mathrm{~mol} \%)$. The desired product was obtained after column chromatography (eluent: pentane/EtOAc) and subsequent preparative TLC (eluent: toluene/EtOAc) as a yellowish sticky oil $(62.9 \mathrm{mg}, 0.14 \mathrm{mmol}, 69 \%,>20: 1 \mathrm{dr}$, $E / Z:>20: 1)$.

$\mathbf{R}_{\mathbf{f}}($ pentane/EtOAc 80:20) $=0.29$.

${ }^{1} \mathbf{H}$ NMR $\left(400 \mathrm{MHz}, \mathrm{CDCl}_{3}\right): \delta(\mathrm{ppm}) 7.38-7.30(\mathrm{~m}, 5 \mathrm{H}), 7.24(\mathrm{dd}, J=8.5,5.8 \mathrm{~Hz}, 1 \mathrm{H}), 7.15(\mathrm{dd}, J=$ $10.0,2.7 \mathrm{~Hz}, 1 \mathrm{H}), 6.95(\mathrm{td}, J=8.3,2.7 \mathrm{~Hz}, 1 \mathrm{H}), 6.65(\mathrm{~d}, J=16.1 \mathrm{~Hz}, 1 \mathrm{H}), 6.38(\mathrm{~d}, J=16.1 \mathrm{~Hz}, 1 \mathrm{H})$, $5.23-5.13(\mathrm{~m}, 2 \mathrm{H}), 4.63(\mathrm{~d}, J=5.4 \mathrm{~Hz}, 1 \mathrm{H}), 4.24-4.08(\mathrm{~m}, 2 \mathrm{H}), 3.96(\mathrm{~s}, 3 \mathrm{H}), 3.27(\mathrm{~d}, J=5.5 \mathrm{~Hz}$, $1 \mathrm{H}), 2.09(\mathrm{~s}, 3 \mathrm{H}), 1.41(\mathrm{~s}, 3 \mathrm{H}), 1.20(\mathrm{t}, J=7.1 \mathrm{~Hz}, 3 \mathrm{H})$.

${ }^{13}$ C NMR (101 MHz, $\left.\mathrm{CDCl}_{3}\right): \delta(\mathrm{ppm}) 172.6,172.6,162.9(\mathrm{~d}, J=247.5 \mathrm{~Hz}), 155.6,137.8(\mathrm{~d}, J=8.0$ $\mathrm{Hz}), 135.6,132.5(\mathrm{~d}, J=3.1 \mathrm{~Hz}), 132.2,130.5(\mathrm{~d}, J=8.5 \mathrm{~Hz}), 129.0(\mathrm{~d}, J=2.3 \mathrm{~Hz}), 128.7,128.4,128.3$, 114.7 (d, $J=21.7 \mathrm{~Hz}), 113.4$ (d, $J=22.4 \mathrm{~Hz}), 74.9,67.2,62.4,62.0,53.3,16.8,16.6,14.1$.

${ }^{19}$ F NMR (376 MHz, $\left.\mathrm{CDCl}_{3}\right): \delta(\mathrm{ppm})-113.0(1 \mathrm{~F})$.

HRMS (ESI): calculated for $\mathrm{C}_{25} \mathrm{H}_{28} \mathrm{FNO}_{6} \mathrm{Na}[\mathrm{M}+\mathrm{Na}]^{+}$: 480.1793; found: 480.1786 .

$( \pm)-1-B e n z y l \quad 4-e t h y l \quad(2 S, 3 S)-3-h y d r o x y-2-((E)-2-((E)-1-(m e t h o x y i m i n o) e t h y l)-5-(($ trifluoromethyl)sulfonyl)oxy)styryl)-2-methylsuccinate (4ea)

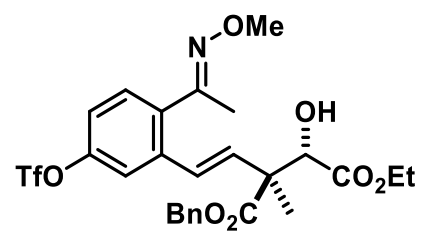

Chemical Formula: $\mathrm{C}_{26} \mathrm{H}_{28} \mathrm{~F}_{3} \mathrm{NO}_{9} \mathrm{~S}$

Exact Mass: 587,1437

Molecular Weight: 587,5632 
Prepared from (E)-4-(1-(methoxyimino)ethyl)phenyl trifluoromethanesulfonate 1e $(59.5 \mathrm{mg}$, $0.20 \mathrm{mmol}, 1.0$ equiv) and benzyl bicyclo[1.1.0]butane-1-carboxylate $\mathbf{2 a}(75.3 \mathrm{mg}, 0.40 \mathrm{mmol}$, 2.0 equiv) according to the general procedure in the presence of $\left[\mathrm{Cp}^{*} \mathrm{Rh}\left(\mathrm{CH}_{3} \mathrm{CN}\right)_{3}\right]\left(\mathrm{SbF}_{6}\right)_{2}(16.7 \mathrm{mg}$, $0.02 \mathrm{mmol}, 10 \mathrm{~mol} \%$ ). The desired product was obtained after column chromatography (eluent: pentane/EtOAc) and subsequent preparative TLC (eluent: toluene/EtOAc) as a yellowish sticky oil (78.8 mg, $0.13 \mathrm{mmol}, 67 \%,>20: 1 \mathrm{dr}, E / Z:>20: 1$ ).

$\mathbf{R}_{\mathbf{f}}($ pentane/EtOAc 80:20) $=0.42$.

${ }^{1} \mathbf{H}$ NMR $\left(400 \mathrm{MHz}, \mathrm{CDCl}_{3}\right): \delta(\mathrm{ppm}) 7.38-7.33(\mathrm{~m}, 6 \mathrm{H}), 7.32(\mathrm{~d}, J=2.6 \mathrm{~Hz}, 1 \mathrm{H}), 7.16(\mathrm{dd}, J=8.5$, $2.6 \mathrm{~Hz}, 1 \mathrm{H}), 6.64(\mathrm{~d}, J=16.2 \mathrm{~Hz}, 1 \mathrm{H}), 6.40(\mathrm{~d}, J=16.3 \mathrm{~Hz}, 1 \mathrm{H}), 5.24-5.13(\mathrm{~m}, 2 \mathrm{H}), 4.63(\mathrm{~s}, 1 \mathrm{H}), 4.24$ - 4.09 (m, 2H), 3.97 (s, 3H), 3.23 (bs, 1H), 2.10 (s, 3H), 1.42 (s, 3H), 1.20 (t, J=7.1 Hz, 3H).

${ }^{13}$ C NMR (101 MHz, $\left.\mathrm{CDCl}_{3}\right): \delta$ (ppm) 172.6, 172.4, 155.0, 149.7, 138.3, 136.5, 135.5, 133.6, 130.7, 128.7, 128.5, 128.4, 128.3, 120.2, 119.6, 118.8 (q, $J=320.8 \mathrm{~Hz}$ ), 74.9, 67.3, 62.5, 62.2, 53.4, 16.7, 16.6, 14.1 .

${ }^{19}$ F NMR $\left(377 \mathrm{MHz}, \mathrm{CDCl}_{3}\right): \delta(\mathrm{ppm})-72.8(3 \mathrm{~F})$.

HRMS (ESI): calculated for $\mathrm{C}_{26} \mathrm{H}_{28} \mathrm{~F}_{3} \mathrm{NO}_{9} \mathrm{SNa}[\mathrm{M}+\mathrm{Na}]^{+}$: 610.1329; found: 610.1325 .

( \pm )-1-Benzyl 4-ethyl (2S,3S)-3-hydroxy-2-((E)-5-(methoxycarbonyl)-2-((E)-1-(methoxyimino)ethyl)styryl)-2-methylsuccinate (4fa)<smiles>CCOC(=O)[C@H](O)[C@@](C)(/C=C/c1cc(C(C)=O)ccc1/C(C)=N/OC)C(=O)O</smiles>

Chemical Formula: $\mathrm{C}_{27} \mathrm{H}_{31} \mathrm{NO}_{8}$

Exact Mass: 497,2050

Molecular Weight: 497,5440

Prepared from methyl (E)-4-(1-(methoxyimino)ethyl)benzoate $\mathbf{1 f}(41.4 \mathrm{mg}, 0.20 \mathrm{mmol}, 1.0$ equiv) and benzyl bicyclo[1.1.0]butane-1-carboxylate $2 \mathrm{a}(75.3 \mathrm{mg}, 0.40 \mathrm{mmol}, 2.0$ equiv) according to the general procedure in the presence of $\left[\mathrm{Cp}^{*} \mathrm{Rh}\left(\mathrm{CH}_{3} \mathrm{CN}\right)_{3}\right]\left(\mathrm{SbF}_{6}\right)_{2}(8.3 \mathrm{mg}, 0.01 \mathrm{mmol}, 5 \mathrm{~mol} \%)$. The desired product was obtained after column chromatography (eluent: pentane/EtOAc) as a yellowish sticky oil (73.3 mg, $0.15 \mathrm{mmol}, 74 \%,>20: 1 \mathrm{dr}, E / Z:>20: 1$ ).

$\mathbf{R}_{\mathbf{f}}($ pentane/EtOAc 75:25) $=0.26$.

${ }^{1} \mathbf{H}$ NMR $\left(400 \mathrm{MHz}, \mathrm{CDCl}_{3}\right): \delta(\mathrm{ppm}) 8.14(\mathrm{~d}, J=1.7 \mathrm{~Hz}, 1 \mathrm{H}), 7.91(\mathrm{dd}, J=8.0,1.7 \mathrm{~Hz}, 1 \mathrm{H}), 7.38-$ $7.28(\mathrm{~m}, 6 \mathrm{H}), 6.69(\mathrm{~d}, J=16.2 \mathrm{~Hz}, 1 \mathrm{H}), 6.48(\mathrm{~d}, J=16.2 \mathrm{~Hz}, 1 \mathrm{H}), 5.18(\mathrm{~s}, 2 \mathrm{H}), 4.64(\mathrm{~d}, J=4.6 \mathrm{~Hz}, 1 \mathrm{H})$, $4.24-4.07$ (m, 2H), 3.97 (s, 3H), $3.92(\mathrm{~s}, 3 \mathrm{H}), 3.29$ (d, $J=5.5 \mathrm{~Hz}, 1 \mathrm{H}), 2.11(\mathrm{~s}, 3 \mathrm{H}), 1.42(\mathrm{~s}, 3 \mathrm{H}), 1.20$ (t, $J=7.1 \mathrm{~Hz}, 3 \mathrm{H})$.

${ }^{13}$ C NMR (101 MHz, $\left.\mathrm{CDCl}_{3}\right): \delta(\mathrm{ppm}) 172.6,172.6,166.7,155.7,140.4,135.8,135.6,132.3,130.4$, 129.0, 128.9, 128.6, 128.6, 128.4, 128.2, 128.1, 75.0, 67.2, 62.4, 62.1, 53.4, 52.3, 16.8, 16.5, 14.1 .

HRMS (ESI): calculated for $\mathrm{C}_{27} \mathrm{H}_{31} \mathrm{NO}_{8} \mathrm{Na}[\mathrm{M}+\mathrm{Na}]^{+}:$520.1942; found: 520.1938 . 

methylsuccinate (4ga)

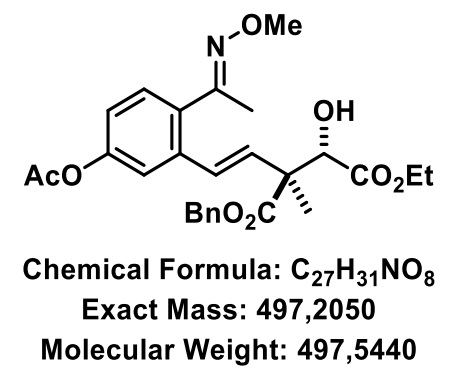

Prepared from methyl (E)-4-(1-(methoxyimino)ethyl)phenyl acetate $1 \mathrm{~g}$ (41.4 mg, $0.20 \mathrm{mmol}, 1.0$ equiv) and benzyl bicyclo[1.1.0]butane-1-carboxylate 2a (75.3 $\mathrm{mg}, 0.40 \mathrm{mmol}, 2.0$ equiv) according to the general procedure in the presence of $\left[\mathrm{Cp}^{*} \mathrm{Rh}\left(\mathrm{CH}_{3} \mathrm{CN}\right)_{3}\right]\left(\mathrm{SbF}_{6}\right)_{2}(8.3 \mathrm{mg}, 0.01 \mathrm{mmol}, 5 \mathrm{~mol} \%)$. The desired product was obtained after column chromatography (eluent: pentane/EtOAc) and subsequent preparative TLC (eluent: toluene/EtOAc) as a yellowish sticky oil $(53.4 \mathrm{mg}, 0.11 \mathrm{mmol}, 54 \%,>20: 1 \mathrm{dr}$, $E / Z:>20: 1)$.

$\mathbf{R}_{\mathbf{f}}($ pentane $/$ EtOAc 65:35) $=0.44$.

${ }^{1} \mathbf{H}$ NMR $\left(400 \mathrm{MHz}, \mathrm{CDCl}_{3}\right): \delta(\mathrm{ppm}) 7.38-7.30(\mathrm{~m}, 5 \mathrm{H}), 7.28(\mathrm{~d}, J=8.4 \mathrm{~Hz}, 1 \mathrm{H}), 7.18(\mathrm{~d}, J=2.4 \mathrm{~Hz}$, 1H), 7.00 (dd, $J=8.4,2.4 \mathrm{~Hz}, 1 \mathrm{H}), 6.66(\mathrm{~d}, J=16.2 \mathrm{~Hz}, 1 \mathrm{H}), 6.36$ (d, $J=16.1 \mathrm{~Hz}, 1 \mathrm{H}), 5.17$ (s, 2H), $4.63(\mathrm{~s}, 1 \mathrm{H}), 4.23-4.07(\mathrm{~m}, 2 \mathrm{H}), 3.95(\mathrm{~s}, 3 \mathrm{H}), 2.30(\mathrm{~s}, 3 \mathrm{H}), 2.10(\mathrm{~s}, 3 \mathrm{H}), 1.40(\mathrm{~s}, 3 \mathrm{H}), 1.19$ (t, $J=7.1$ $\mathrm{Hz}, 3 \mathrm{H})$, one proton signal missing due to broadening.

${ }^{13}$ C NMR (101 MHz, $\left.\mathrm{CDCl}_{3}\right): \delta$ (ppm) 172.7, 172.6, 169.3, 155.7, 151.0, 137.1, 135.7, 134.0, 131.9, $129.9,129.2,128.6,128.4,128.3,121.0,119.8,74.9,67.2,62.4,62.0,53.3,21.2,16.7,14.1$, one carbon signal missing due to overlap.

HRMS (ESI): calculated for $\mathrm{C}_{27} \mathrm{H}_{31} \mathrm{NO}_{8} \mathrm{Na}[\mathrm{M}+\mathrm{Na}]^{+}:$520.1942; found: 520.1937 .

( \pm )-1-Benzyl 4-ethyl (2S,3S)-3-hydroxy-2-((E)-5-methoxy-2-((E)-1-(methoxyimino)ethyl)styryl)-2methylsuccinate (4ha)<smiles>CCOC(=O)[C@H](O)C(C)(C)/C=C/c1cc(OC)ccc1/C(C)=N/OC</smiles>

Chemical Formula: $\mathrm{C}_{26} \mathrm{H}_{31} \mathrm{NO}_{7}$

Exact Mass: 469,2101

Molecular Weight: 469,5340

Prepared from methyl (E)-1-(4-methoxyphenyl)ethan-1-one $O$-methyl oxime $\mathbf{1 h}$ (35.8 $\mathrm{mg}, 0.20 \mathrm{mmol}$, 1.0 equiv) and benzyl bicyclo[1.1.0]butane-1-carboxylate $2 \mathrm{a}(75.3 \mathrm{mg}, 0.40 \mathrm{mmol}, 2.0$ equiv) according to the general procedure in the presence of $\left[\mathrm{Cp}^{*} \mathrm{Rh}\left(\mathrm{CH}_{3} \mathrm{CN}\right)_{3}\right]\left(\mathrm{SbF}_{6}\right)_{2}(8.3 \mathrm{mg}, 0.01 \mathrm{mmol}, 5 \mathrm{~mol} \%)$. The desired product was obtained after column chromatography (eluent: pentane/EtOAc) as a yellowish sticky oil (75.1 mg, $0.16 \mathrm{mmol}, 80 \%,>20: 1 \mathrm{dr}, E / Z:>20: 1)$.

$\mathbf{R}_{\mathbf{f}}($ pentane/EtOAc 75:25) $=0.31$.

${ }^{1} \mathbf{H}$ NMR $\left(400 \mathrm{MHz}, \mathrm{CDCl}_{3}\right): \delta(\mathrm{ppm}) 7.38-7.29(\mathrm{~m}, 5 \mathrm{H}), 7.20(\mathrm{~d}, J=8.5 \mathrm{~Hz}, 1 \mathrm{H}), 6.97(\mathrm{~d}, J=2.7 \mathrm{~Hz}$, $1 \mathrm{H}), 6.80(\mathrm{dd}, J=8.5,2.7 \mathrm{~Hz}, 1 \mathrm{H}), 6.71(\mathrm{~d}, J=16.1 \mathrm{~Hz}, 1 \mathrm{H}), 6.37$ (d, $J=16.2 \mathrm{~Hz}, 1 \mathrm{H}), 5.22-5.14$ (m, 
$2 \mathrm{H}), 4.65(\mathrm{~d}, J=4.2 \mathrm{~Hz}, 1 \mathrm{H}), 4.23-4.07(\mathrm{~m}, 2 \mathrm{H}), 3.95(\mathrm{~s}, 3 \mathrm{H}), 3.80(\mathrm{~s}, 3 \mathrm{H}), 3.29(\mathrm{~d}, J=5.5 \mathrm{~Hz}, 1 \mathrm{H})$, $2.10(\mathrm{~s}, 3 \mathrm{H}), 1.42(\mathrm{~s}, 3 \mathrm{H}), 1.20(\mathrm{t}, J=7.2 \mathrm{~Hz}, 3 \mathrm{H})$.

${ }^{13} \mathrm{C}$ NMR (101 MHz, $\left.\mathrm{CDCl}_{3}\right): \delta$ (ppm) 172.8, 172.6, 159.9, 156.2, 136.9, 135.7, 131.0, 130.1, 130.0, $129.2,128.6,128.3,128.2,113.5,111.9,75.0,67.1,62.3,61.8,55.4,53.2,16.8,16.7,14.1$.

HRMS (ESI): calculated for $\mathrm{C}_{26} \mathrm{H}_{31} \mathrm{NO}_{7} \mathrm{Na}[\mathrm{M}+\mathrm{Na}]^{+}: 492.1993$; found: 492.1985 .

( \pm -1-Benzyl 4-ethyl (2S,3S)-3-hydroxy-2-((E)-5-hydroxy-2-((E)-1-(methoxyimino)ethyl)styryl)-2methylsuccinate (4ia)

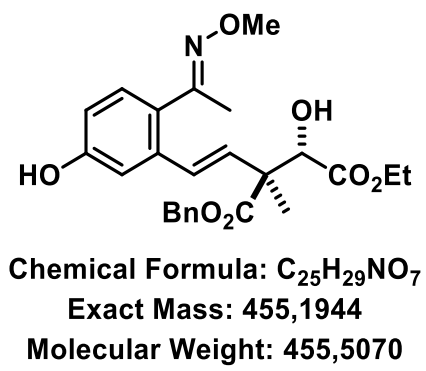

Prepared from methyl (E)-1-(4-hydroxyphenyl)ethan-1-one $O$-methyl oxime 1i (33.0 mg, $0.20 \mathrm{mmol}$, 1.0 equiv) and benzyl bicyclo[1.1.0]butane-1-carboxylate $2 \mathrm{a}(75.3 \mathrm{mg}, 0.40 \mathrm{mmol}, 2.0$ equiv) according to the general procedure in the presence of $\left[\mathrm{Cp} * \mathrm{Rh}\left(\mathrm{CH}_{3} \mathrm{CN}\right)_{3}\right]\left(\mathrm{SbF}_{6}\right)_{2}(8.3 \mathrm{mg}, 0.01 \mathrm{mmol}, 5 \mathrm{~mol} \%)$. The desired product was obtained after column chromatography (eluent: pentane/EtOAc) and subsequent preparative TLC (eluent: toluene/EtOAc) as a yellowish sticky oil (53.0 mg, $0.11 \mathrm{mmol}$, $57 \%,>20: 1 \mathrm{dr}, E / Z:>20: 1)$.

$\mathbf{R}_{\mathbf{f}}($ pentane/EtOAc 60:40) $=0.31$.

${ }^{1} \mathbf{H}$ NMR $\left(400 \mathrm{MHz}, \mathrm{CDCl}_{3}\right): \delta(\mathrm{ppm}) 7.38-7.29(\mathrm{~m}, 6 \mathrm{H}), 7.08(\mathrm{~d}, J=8.4 \mathrm{~Hz}, 1 \mathrm{H}), 6.84(\mathrm{~d}, J=2.5 \mathrm{~Hz}$, $1 \mathrm{H}), 6.68-6.60(\mathrm{~m}, 2 \mathrm{H}), 6.29(\mathrm{~d}, J=16.1 \mathrm{~Hz}, 1 \mathrm{H}), 5.16(\mathrm{~s}, 2 \mathrm{H}), 4.68(\mathrm{~s}, 1 \mathrm{H}), 4.20-4.08(\mathrm{~m}, 2 \mathrm{H}), 3.94$ $(\mathrm{s}, 3 \mathrm{H}), 2.08(\mathrm{~s}, 3 \mathrm{H}), 1.39(\mathrm{~s}, 3 \mathrm{H}), 1.19(\mathrm{t}, J=7.1 \mathrm{~Hz}, 3 \mathrm{H})$, one proton signal missing due to broadening.

${ }^{13} \mathrm{C}$ NMR (101 MHz, $\left.\mathrm{CDCl}_{3}\right): \delta$ (ppm) 173.1, 172.5, 156.8, 156.5, 136.9, 135.7, 130.9, 130.1, 130.0, $128.7,128.6,128.4,128.3,115.2,113.6,75.1,67.3,62.4,61.9,53.3,17.0,16.4,14.2$.

HRMS (ESI): calculated for $\mathrm{C}_{25} \mathrm{H}_{29} \mathrm{NO}_{7} \mathrm{Na}[\mathrm{M}+\mathrm{Na}]^{+}$: 478.1836; found: 478.1831.

( \pm )-1-Benzyl 4-ethyl $(2 S, 3 S)-2-((E)-5-(($ tert-butoxycarbonyl)amino)-2-((E)-1 (methoxyimino)ethyl)styryl)-3-hydroxy-2-methylsuccinate ( $4 \mathrm{ja})$

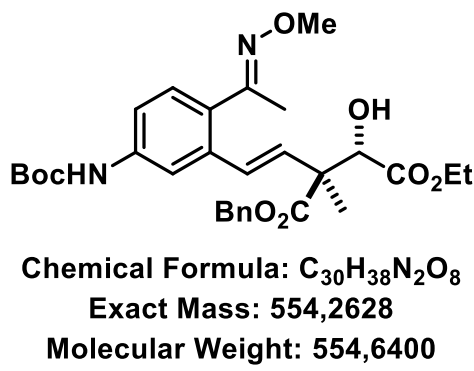

Prepared from tert-butyl (E)-(4-(1-(methoxyimino)ethyl)phenyl)carbamate 1j $(52.9 \mathrm{mg}, 0.20 \mathrm{mmol}$, 1.0 equiv) and benzyl bicyclo[1.1.0]butane-1-carboxylate $2 \mathrm{a}(75.3 \mathrm{mg}, 0.40 \mathrm{mmol}, 2.0$ equiv) according to the general procedure in the presence of $\left[\mathrm{Cp}^{*} \mathrm{Rh}\left(\mathrm{CH}_{3} \mathrm{CN}\right)_{3}\right]\left(\mathrm{SbF}_{6}\right)_{2}(8.3 \mathrm{mg}, 0.01 \mathrm{mmol}, 5 \mathrm{~mol} \%)$. 
The desired product was obtained after column chromatography (eluent: pentane/EtOAc) and subsequent preparative TLC (eluent: toluene/EtOAc) as a yellowish sticky oil (68.9 $\mathrm{mg}, 0.12 \mathrm{mmol}$, $62 \%,>20: 1 \mathrm{dr}, E / Z:>20: 1)$.

$\mathbf{R}_{\mathbf{f}}($ pentane $/$ EtOAc $70: 30)=0.31$.

${ }^{1} \mathbf{H}$ NMR $\left(400 \mathrm{MHz}, \mathrm{CDCl}_{3}\right): \delta(\mathrm{ppm}) 7.41-7.27(\mathrm{~m}, 7 \mathrm{H}), 7.20(\mathrm{~d}, J=8.4 \mathrm{~Hz}, 1 \mathrm{H}), 6.68(\mathrm{~d}, J=16.2$ $\mathrm{Hz}, 1 \mathrm{H}), 6.60(\mathrm{~s}, 1 \mathrm{H}), 6.33(\mathrm{~d}, J=16.2 \mathrm{~Hz}, 1 \mathrm{H}), 5.22-5.11(\mathrm{~m}, 2 \mathrm{H}), 4.63(\mathrm{~s}, 1 \mathrm{H}), 4.24-4.08(\mathrm{~m}, 2 \mathrm{H})$, $3.94(\mathrm{~s}, 3 \mathrm{H}), 3.25$ (bs, 1H), $2.08(\mathrm{~s}, 3 \mathrm{H}), 1.51$ (s, 9H), 1.40 (s, 3H), 1.19 (t, $J=7.1 \mathrm{~Hz}, 3 \mathrm{H})$.

${ }^{13}$ C NMR (101 MHz, $\left.\mathrm{CDCl}_{3}\right): \delta$ (ppm) 172.8, 172.6, 156.1, 152.6, 138.9, 136.3, 135.8, 131.1, 131.1, 130.0, 129.5, 128.7, 128.4, 117.7, 116.5, 80.8, 75.0, 67.1, 62.4, 61.9, 53.3, 28.4, 16.7, 16.6, 14.2, one carbon signal missing due to overlap.

HRMS (ESI): calculated for $\mathrm{C}_{30} \mathrm{H}_{38} \mathrm{~N}_{2} \mathrm{O}_{8} \mathrm{Na}[\mathrm{M}+\mathrm{Na}]^{+}:$577.2520; found: 577.2520.

( \pm -1-Benzyl 4-ethyl (2S,3S)-2-((E)-5-cyclohexyl-2-((E)-1-(methoxyimino)ethyl)styryl)-3-hydroxy2-methylsuccinate (4ka)<smiles>CCOC(O)[C@H](O)C(C)(C)[14C](=O)c1cc(C2CCCCC2)ccc1/C(C)=N/OC</smiles>

Chemical Formula: $\mathrm{C}_{31} \mathrm{H}_{39} \mathrm{NO}_{6}$

Exact Mass: 521,2777

Molecular Weight: 521,6540

Prepared from (E)-1-(4-cyclohexylphenyl)ethan-1-one $O$-methyl oxime 1k $(46.3 \mathrm{mg}, 0.20 \mathrm{mmol}$, 1.0 equiv) and benzyl bicyclo[1.1.0]butane-1-carboxylate $2 \mathrm{a}(75.3 \mathrm{mg}, 0.40 \mathrm{mmol}, 2.0$ equiv) according to the general procedure in the presence of $\left[\mathrm{Cp}^{*} \mathrm{Rh}\left(\mathrm{CH}_{3} \mathrm{CN}\right)_{3}\right]\left(\mathrm{SbF}_{6}\right)_{2}(8.3 \mathrm{mg}, 0.01 \mathrm{mmol}, 5 \mathrm{~mol} \%)$. The desired product was obtained after column chromatography (eluent: pentane/EtOAc) and subsequent preparative TLC (eluent: toluene/EtOAc) as a yellowish sticky oil $(65.1 \mathrm{mg}, 0.12 \mathrm{mmol}$, $62 \%,>20: 1 \mathrm{dr}, E / Z:>20: 1)$.

$\mathbf{R}_{\mathbf{f}}($ pentane/EtOAc 85:15) $=0.22$.

${ }^{1} \mathbf{H}$ NMR (400 MHz, $\left.\mathrm{CDCl}_{3}\right): \delta(\mathrm{ppm}) 7.39-7.30(\mathrm{~m}, 5 \mathrm{H}), 7.28(\mathrm{~d}, J=1.7 \mathrm{~Hz}, 1 \mathrm{H}), 7.19(\mathrm{~d}, J=7.9 \mathrm{~Hz}$, $1 \mathrm{H}), 7.11(\mathrm{dd}, J=7.9,1.8 \mathrm{~Hz}, 1 \mathrm{H}), 6.70(\mathrm{~d}, J=16.2 \mathrm{~Hz}, 1 \mathrm{H}), 6.36(\mathrm{~d}, J=16.2 \mathrm{~Hz}, 1 \mathrm{H}), 5.25-5.13$ (m, 2H), $4.66(\mathrm{~s}, 1 \mathrm{H}), 4.24-4.09(\mathrm{~m}, 2 \mathrm{H}), 3.95(\mathrm{~s}, 3 \mathrm{H}), 2.89(\mathrm{bs}, 1 \mathrm{H}), 2.55-2.44(\mathrm{~m}, 1 \mathrm{H}), 2.11(\mathrm{~s}, 3 \mathrm{H})$, $1.90-1.80(\mathrm{~m}, 4 \mathrm{H}), 1.79-1.73(\mathrm{~m}, 1 \mathrm{H}), 1.44-1.37(\mathrm{~m}, 7 \mathrm{H}), 1.32-1.24(\mathrm{~m}, 1 \mathrm{H}), 1.21(\mathrm{t}, J=7.1 \mathrm{~Hz}$, $3 \mathrm{H})$.

${ }^{13}$ C NMR (101 MHz, $\left.\mathrm{CDCl}_{3}\right): \delta$ (ppm) 173.0, 172.7, 156.6, 148.9, 135.8, 135.2, 134.0, 130.5, 130.4, 128.7, 128.6, 128.4, 128.3, 126.3, 125.5, 75.0, 67.1, 62.3, 61.9, 53.3, 44.6, 34.4, 34.4, 26.9, 26.2, 16.7, $16.7,14.2$, one carbon signal missing due to overlap.

HRMS (ESI): calculated for $\mathrm{C}_{31} \mathrm{H}_{39} \mathrm{NO}_{6} \mathrm{Na}$ [M+Na] $]^{+}: 544.2670$; found: 544.2672. 


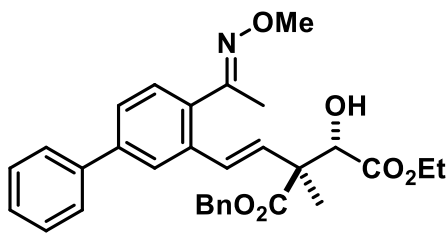

Chemical Formula: $\mathrm{C}_{31} \mathrm{H}_{33} \mathrm{NO}_{6}$

Exact Mass: 515,2308

Molecular Weight: $\mathbf{5 1 5 , 6 0 6 0}$

Prepared from $(E)-1-\left(\left[1,1^{\prime}\right.\right.$-biphenyl]-4-yl)ethan-1-one $O$-methyl oxime 11 (45.1 mg, $0.20 \mathrm{mmol}$, 1.0 equiv) and benzyl bicyclo[1.1.0]butane-1-carboxylate $2 \mathbf{a}(75.3 \mathrm{mg}, 0.40 \mathrm{mmol}, 2.0$ equiv) according to the general procedure in the presence of $\left[\mathrm{Cp}^{*} \mathrm{Rh}\left(\mathrm{CH}_{3} \mathrm{CN}\right)_{3}\right]\left(\mathrm{SbF}_{6}\right)_{2}(8.3 \mathrm{mg}, 0.01 \mathrm{mmol}, 5 \mathrm{~mol} \%)$. The desired product was obtained after column chromatography (eluent: pentane/EtOAc) and subsequent preparative TLC (eluent: toluene/EtOAc) as a yellowish sticky oil ( $78.6 \mathrm{mg}, 0.15 \mathrm{mmol}$, $76 \%,>20: 1 \mathrm{dr}, E / Z:>20: 1)$.

$\mathbf{R}_{\mathbf{f}}($ pentane $/$ EtOAc 80:20) $=0.29$.

${ }^{1} \mathbf{H}$ NMR $\left(400 \mathrm{MHz}, \mathrm{CDCl}_{3}\right): \delta(\mathrm{ppm}) 7.66(\mathrm{~d}, J=1.9 \mathrm{~Hz}, 1 \mathrm{H}), 7.60-7.56(\mathrm{~m}, 2 \mathrm{H}), 7.49(\mathrm{dd}, J=8.0$, $1.9 \mathrm{~Hz}, 1 \mathrm{H}), 7.47-7.43(\mathrm{~m}, 2 \mathrm{H}), 7.40-7.29(\mathrm{~m}, 7 \mathrm{H}), 6.77$ (d, $J=16.2 \mathrm{~Hz}, 1 \mathrm{H}), 6.45(\mathrm{~d}, J=16.1 \mathrm{~Hz}$, 1H), $5.25-5.14(\mathrm{~m}, 2 \mathrm{H}), 4.67(\mathrm{~s}, 1 \mathrm{H}), 4.25-4.09(\mathrm{~m}, 2 \mathrm{H}), 3.99(\mathrm{~s}, 3 \mathrm{H}), 2.16(\mathrm{~s}, 3 \mathrm{H}), 1.44(\mathrm{~s}, 3 \mathrm{H}), 1.20$ (t, $J=7.2 \mathrm{~Hz}, 3 \mathrm{H})$, one proton signal missing due to broadening.

${ }^{13}$ C NMR (101 MHz, $\left.\mathrm{CDCl}_{3}\right): \delta(\mathrm{ppm}) 172.8,172.6,156.2,141.8,140.6,135.9,135.7,135.3,131.3$, $130.0,129.1,128.9,128.6,128.3,128.2,127.7,127.3,126.5,125.7,75.0,67.1,62.3,62.0,53.3,16.7$, 14.1 , one carbon signal missing due to overlap.

HRMS (ESI): calculated for $\mathrm{C}_{31} \mathrm{H}_{33} \mathrm{NO}_{6} \mathrm{Na}[\mathrm{M}+\mathrm{Na}]^{+}:$538.2200; found: 538.2195.

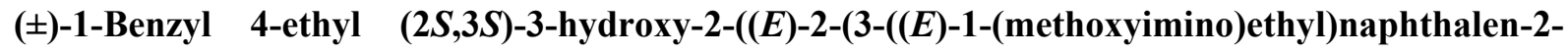
yl)vinyl)-2-methylsuccinate (4ma)<smiles>CCOC(=O)[C@H](O)C(C)(C)/C=C/c1cc2ccccc2cc1/C(C)=N/OC</smiles>

Chemical Formula: $\mathrm{C}_{29} \mathrm{H}_{31} \mathrm{NO}_{6}$

Exact Mass: 489,2151

Molecular Weight: 489,5680

Prepared from (E)-1-naphthalen-2-yl)ethan-1-one $O$-methyl oxime 1m (39.9 mg, $0.20 \mathrm{mmol}$, 1.0 equiv) and benzyl bicyclo[1.1.0]butane-1-carboxylate $\mathbf{2 a}(75.3 \mathrm{mg}, 0.40 \mathrm{mmol}, 2.0$ equiv) according to the general procedure in the presence of $\left[\mathrm{Cp}^{*} \mathrm{Rh}\left(\mathrm{CH}_{3} \mathrm{CN}\right)_{3}\right]\left(\mathrm{SbF}_{6}\right)_{2}(8.3 \mathrm{mg}, 0.01 \mathrm{mmol}, 5 \mathrm{~mol} \%)$. The desired product was obtained after column chromatography (eluent: pentane/EtOAc) and subsequent preparative TLC (eluent: toluene/EtOAc) as a yellowish sticky oil (55.2 $\mathrm{mg}, 0.11 \mathrm{mmol}$, $56 \%,>20: 1 \mathrm{dr},>20: 1$ site selectivity, $E / Z:>20: 1)$.

$\mathbf{R}_{\mathbf{f}}($ pentane/EtOAc 70:30) $=0.60$.

${ }^{1} \mathbf{H}$ NMR $\left(400 \mathrm{MHz}, \mathrm{CDCl}_{3}\right): \delta(\mathrm{ppm}) 7.88(\mathrm{~s}, 1 \mathrm{H}), 7.82-7.77(\mathrm{~m}, 2 \mathrm{H}), 7.76(\mathrm{~s}, 1 \mathrm{H}), 7.51-7.42(\mathrm{~m}$, 2H), $7.41-7.30(\mathrm{~m}, 5 \mathrm{H}), 6.78(\mathrm{~d}, J=16.1 \mathrm{~Hz}, 1 \mathrm{H}), 6.49(\mathrm{~d}, J=16.1 \mathrm{~Hz}, 1 \mathrm{H}), 5.34-5.09(\mathrm{~m}, 2 \mathrm{H}), 4.70$ 
$(\mathrm{d}, J=5.0 \mathrm{~Hz}, 1 \mathrm{H}), 4.28-4.09(\mathrm{~m}, 2 \mathrm{H}), 4.01(\mathrm{~s}, 3 \mathrm{H}), 3.26(\mathrm{~d}, J=5.6 \mathrm{~Hz}, 1 \mathrm{H}), 2.18(\mathrm{~s}, 3 \mathrm{H}), 1.47(\mathrm{~s}$, $3 \mathrm{H}), 1.22(\mathrm{t}, J=7.1 \mathrm{~Hz}, 3 \mathrm{H})$.

${ }^{13}$ C NMR (101 MHz, $\left.\mathrm{CDCl}_{3}\right): \delta$ (ppm) 172.9, 172.7, 156.8, 135.8, 134.7, 133.5, 133.4, 132.6, 131.3, $130.3,128.7,128.4,128.3,128.0,128.0,127.8,126.9,126.4,126.2,75.1,67.2,62.4,62.0,53.4,17.0$, $16.7,14.2$.

HRMS (ESI): calculated for $\mathrm{C}_{29} \mathrm{H}_{31} \mathrm{NO}_{6} \mathrm{Na}[\mathrm{M}+\mathrm{Na}]^{+}:$512.2044; found: 512.2041 .

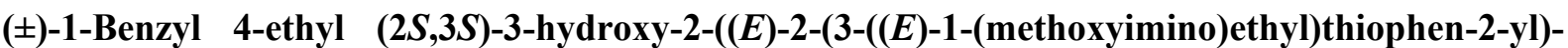
vinyl)-2-methylsuccinate (4na)

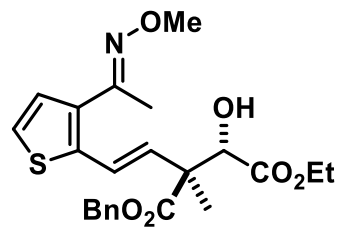

Chemical Formula: $\mathrm{C}_{23} \mathrm{H}_{27} \mathrm{NO}_{6} \mathrm{~S}$

Exact Mass: 445,1559

Molecular Weight: 445,5300

Prepared from $(E)$-1-(thiophen-3-yl)ethan-1-one $O$-methyl oxime 1 n $(31.0 \mathrm{mg}, 0.20 \mathrm{mmol}, 1.0$ equiv) and benzyl bicyclo[1.1.0]butane-1-carboxylate $2 \mathrm{a}(75.3 \mathrm{mg}, 0.40 \mathrm{mmol}, 2.0$ equiv) according to the general procedure in the presence of $\left[\mathrm{Cp}^{*} \mathrm{Rh}\left(\mathrm{CH}_{3} \mathrm{CN}\right)_{3}\right]\left(\mathrm{SbF}_{6}\right)_{2}(8.3 \mathrm{mg}, 0.01 \mathrm{mmol}, 5 \mathrm{~mol} \%)$. The desired product was obtained after column chromatography (eluent: pentane/EtOAc) and subsequent preparative TLC (eluent: toluene/EtOAc) as a yellowish sticky oil $(55.5 \mathrm{mg}, 0.12 \mathrm{mmol}, 62 \%,>20: 1 \mathrm{dr}$, $>20: 1$ site selectivity, $E / Z:>20: 1)$.

$\mathbf{R}_{\mathbf{f}}($ pentane/EtOAc 80:20) $=0.27$.

${ }^{1} \mathbf{H}$ NMR $\left(400 \mathrm{MHz}, \mathrm{CDCl}_{3}\right): \delta(\mathrm{ppm}) 7.37-7.30(\mathrm{~m}, 5 \mathrm{H}), 7.16(\mathrm{~d}, J=16.3 \mathrm{~Hz}, 1 \mathrm{H}), 7.10(\mathrm{~d}, J=5.3$ $\mathrm{Hz}, 1 \mathrm{H}), 7.01(\mathrm{~d}, J=5.3 \mathrm{~Hz}, 1 \mathrm{H}), 6.39(\mathrm{~d}, J=16.2 \mathrm{~Hz}, 1 \mathrm{H}), 5.24-5.12(\mathrm{~m}, 2 \mathrm{H}), 4.58(\mathrm{~s}, 1 \mathrm{H}), 4.24-$ $4.07(\mathrm{~m}, 2 \mathrm{H}), 3.95(\mathrm{~s}, 3 \mathrm{H}), 3.13(\mathrm{bs}, 1 \mathrm{H}), 2.16(\mathrm{~s}, 3 \mathrm{H}), 1.43(\mathrm{~s}, 3 \mathrm{H}), 1.20(\mathrm{t}, J=7.1 \mathrm{~Hz}, 3 \mathrm{H})$.

${ }^{13} \mathbf{C}$ NMR (101 MHz, $\left.\mathrm{CDCl}_{3}\right): \delta(\mathrm{ppm})$ 172.7, 172.6, 152.0, 139.3, 135.7, 134.8, 130.1, 128.6, 128.3, $128.2,128.1,124.9,123.6,75.1,67.1,62.4,62.1,53.4,16.7,15.4,14.2$.

HRMS (ESI): calculated for $\mathrm{C}_{23} \mathrm{H}_{27} \mathrm{NO}_{6} \mathrm{SNa}[\mathrm{M}+\mathrm{Na}]^{+}$: 468.1451; found: 468.1446.

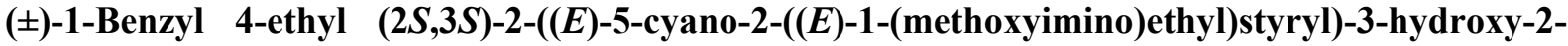
methylsuccinate (40a)

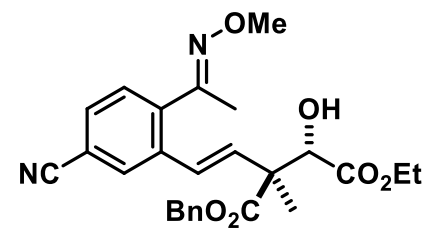

Chemical Formula: $\mathrm{C}_{26} \mathrm{H}_{28} \mathrm{~N}_{2} \mathrm{O}_{6}$

Exact Mass: 464,1947

Molecular Weight: 464,5180

Prepared from (E)-4-(1-(methoxyimino)ethyl)benzonitrile $10(34.8 \mathrm{mg}, 0.20 \mathrm{mmol}, 1.0$ equiv) and benzyl bicyclo[1.1.0]butane-1-carboxylate $\mathbf{2 a}(75.3 \mathrm{mg}, 0.40 \mathrm{mmol}, 2.0$ equiv) according to the general 
procedure in the presence of $\left[\mathrm{Cp} * \mathrm{Rh}\left(\mathrm{CH}_{3} \mathrm{CN}\right)_{3}\right]\left(\mathrm{SbF}_{6}\right)_{2}(16.7 \mathrm{mg}, 0.02 \mathrm{mmol}, 10 \mathrm{~mol} \%)$. The desired product was obtained after column chromatography (eluent: pentane/EtOAc) as an orange sticky oil (63.9 mg, $0.14 \mathrm{mmol}, 69 \%,>20: 1 \mathrm{dr}, E / Z:>20: 1)$.

$\mathbf{R}_{\mathbf{f}}($ pentane/EtOAc 75:25) $=0.27$.

${ }^{1} \mathbf{H}$ NMR $\left(400 \mathrm{MHz}, \mathrm{CDCl}_{3}\right): \delta(\mathrm{ppm}) 7.69(\mathrm{~d}, J=1.6 \mathrm{~Hz}, 1 \mathrm{H}), 7.52(\mathrm{dd}, J=8.0,1.6 \mathrm{~Hz}, 1 \mathrm{H}), 7.40-$ $7.32(\mathrm{~m}, 6 \mathrm{H}), 6.63(\mathrm{~d}, J=16.3 \mathrm{~Hz}, 1 \mathrm{H}), 6.40(\mathrm{~d}, J=16.3 \mathrm{~Hz}, 1 \mathrm{H}), 5.24-5.14(\mathrm{~m}, 2 \mathrm{H}), 4.65(\mathrm{~s}, 1 \mathrm{H})$, $4.25-4.08(\mathrm{~m}, 2 \mathrm{H}), 3.97(\mathrm{~s}, 3 \mathrm{H}), 2.10(\mathrm{~s}, 3 \mathrm{H}), 1.40(\mathrm{~s}, 3 \mathrm{H}), 1.21(\mathrm{t}, J=7.1 \mathrm{~Hz}, 3 \mathrm{H})$, one proton signal missing due to broadening.

${ }^{13}$ C NMR (101 MHz, $\left.\mathrm{CDCl}_{3}\right): \delta$ (ppm) 172.5, 172.4, 154.9, 140.4, 136.9, 135.5, 133.7, 130.8, 130.8, $129.5,128.7,128.6,128.4,128.2,118.5,112.7,74.8,67.3,62.5,62.3,53.4,16.5,16.3,14.2$.

HRMS (ESI): calculated for $\mathrm{C}_{26} \mathrm{H}_{28} \mathrm{~N}_{2} \mathrm{O}_{6} \mathrm{Na}[\mathrm{M}+\mathrm{Na}]^{+}$: 487.1840; found: 487.1832 .

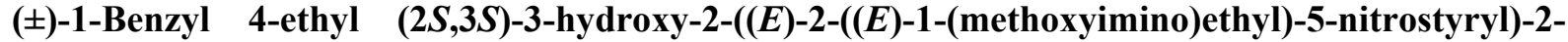 methylsuccinate (4pa)}<smiles>CCOC(=O)[C@H](O)C(C)(C)/C=C/c1cc([N+](=O)[O-])ccc1/C(C)=N/OC</smiles>

Chemical Formula: $\mathrm{C}_{25} \mathrm{H}_{28} \mathrm{~N}_{2} \mathrm{O}_{8}$

Exact Mass: 484,1846

Molecular Weight: 484,5050

Prepared from (E)-1-(4-nitrophenyl)ethan-1-one $O$-methyl oxime 1p (38.8 mg, $0.20 \mathrm{mmol}, 1.0$ equiv) and benzyl bicyclo[1.1.0]butane-1-carboxylate 2a (75.3 $\mathrm{mg}, 0.40 \mathrm{mmol}, 2.0$ equiv) according to the general procedure in the presence of $\left[\mathrm{Cp}^{*} \mathrm{Rh}\left(\mathrm{CH}_{3} \mathrm{CN}\right)_{3}\right]\left(\mathrm{SbF}_{6}\right)_{2}(8.3 \mathrm{mg}, 0.01 \mathrm{mmol}, 5 \mathrm{~mol} \%)$. The desired product was obtained after column chromatography (eluent: pentane/EtOAc) and subsequent preparative TLC (eluent: toluene/EtOAc) as a yellowish sticky oil $(51.3 \mathrm{mg}, 0.11 \mathrm{mmol}, 53 \%,>20: 1 \mathrm{dr}$, $E / Z:>20: 1)$.

$\mathbf{R}_{\mathbf{f}}($ pentane/EtOAc 70:30) $=0.40$.

${ }^{1} \mathbf{H}$ NMR $\left(400 \mathrm{MHz}, \mathrm{CDCl}_{3}\right): \delta(\mathrm{ppm}) 8.30(\mathrm{~d}, J=2.4 \mathrm{~Hz}, 1 \mathrm{H}), 8.08(\mathrm{dd}, J=8.4,2.3 \mathrm{~Hz}, 1 \mathrm{H}), 7.44(\mathrm{~d}$, $J=8.5 \mathrm{~Hz}, 1 \mathrm{H}), 7.40-7.28(\mathrm{~m}, 5 \mathrm{H}), 6.68(\mathrm{~d}, J=16.2 \mathrm{~Hz}, 1 \mathrm{H}), 6.53(\mathrm{~d}, J=16.2 \mathrm{~Hz}, 1 \mathrm{H}), 5.26-5.13$ $(\mathrm{m}, 2 \mathrm{H}), 4.65(\mathrm{~d}, J=4.4 \mathrm{~Hz}, 1 \mathrm{H}), 4.27-4.08(\mathrm{~m}, 2 \mathrm{H}), 3.99(\mathrm{~s}, 3 \mathrm{H}), 3.28(\mathrm{~d}, J=5.2 \mathrm{~Hz}, 1 \mathrm{H}), 2.12(\mathrm{~s}$, $3 \mathrm{H}), 1.43(\mathrm{~s}, 3 \mathrm{H}), 1.21(\mathrm{t}, J=7.1 \mathrm{~Hz}, 3 \mathrm{H})$.

${ }^{13} \mathrm{C}$ NMR (101 MHz, $\left.\mathrm{CDCl}_{3}\right): \delta$ (ppm) 172.5, 172.3, 154.7, 148.0, 142.0, 137.3, 135.5, 134.1, 129.9, $128.7,128.5,128.4,128.1,122.2,121.9,74.8,67.3,62.6,62.3,53.4,16.7,16.4,14.2$.

HRMS (ESI): calculated for $\mathrm{C}_{25} \mathrm{H}_{28} \mathrm{~N}_{2} \mathrm{O}_{8} \mathrm{Na}[\mathrm{M}+\mathrm{Na}]^{+}$: 507.1738; found: 507.1734. 
( \pm )-1-Benzyl 4-ethyl (2S,3S)-3-hydroxy-2-((E)-2-((E)-1-(methoxyimino)ethyl)-5-(trifluoromethyl)styryl)-2-methylsuccinate (4qa)<smiles>CCOC(O)[C@H](O)C(C)(C)/C=C/c1cc(C(F)(F)F)ccc1/C(C)=N/OC</smiles>

Chemical Formula: $\mathrm{C}_{26} \mathrm{H}_{28} \mathrm{~F}_{3} \mathrm{NO}_{6}$

Exact Mass: 507,1869

Molecular Weight: $\mathbf{5 0 7 , 5 0 6 2}$

Prepared from (E)-1-(4-(trifluoromethyl)phenyl)ethan-1-one $O$-methyl oxime 1q (43.4 mg, $0.20 \mathrm{mmol}$, 1.0 equiv) and benzyl bicyclo[1.1.0]butane-1-carboxylate $2 \mathrm{a}(75.3 \mathrm{mg}, 0.40 \mathrm{mmol}, 2.0$ equiv) according to the general procedure in the presence of $\left[\mathrm{Cp}^{*} \mathrm{Rh}\left(\mathrm{CH}_{3} \mathrm{CN}\right)_{3}\right]\left(\mathrm{SbF}_{6}\right)_{2}(8.3 \mathrm{mg}, 0.01 \mathrm{mmol}, 5 \mathrm{~mol} \%)$. The desired product was obtained after column chromatography (eluent: pentane/EtOAc) as a yellowish sticky oil (60.2 mg, $0.12 \mathrm{mmol}, 59 \%,>20: 1 \mathrm{dr}, E / Z:>20: 1)$.

$\mathbf{R}_{\mathbf{f}}($ pentane $/$ EtOAc 80:20) $=0.33$.

${ }^{1} \mathbf{H}$ NMR $\left(400 \mathrm{MHz}, \mathrm{CDCl}_{3}\right): \delta(\mathrm{ppm}) 7.70-7.67(\mathrm{~m}, 1 \mathrm{H}), 7.53-7.48(\mathrm{~m}, 1 \mathrm{H}), 7.41-7.30(\mathrm{~m}, 6 \mathrm{H})$, $6.69(\mathrm{~d}, J=16.3 \mathrm{~Hz}, 1 \mathrm{H}), 6.45(\mathrm{~d}, J=16.2 \mathrm{~Hz}, 1 \mathrm{H}), 5.25-5.12(\mathrm{~m}, 2 \mathrm{H}), 4.64(\mathrm{~d}, J=5.3 \mathrm{~Hz}, 1 \mathrm{H}), 4.24$ -4.09 (m, 2H), $3.98(\mathrm{~s}, 3 \mathrm{H}), 3.28(\mathrm{~d}, J=5.5 \mathrm{~Hz}, 1 \mathrm{H}), 2.12(\mathrm{~s}, 3 \mathrm{H}), 1.43(\mathrm{~s}, 3 \mathrm{H}), 1.21(\mathrm{t}, J=7.1 \mathrm{~Hz}, 3 \mathrm{H})$.

${ }^{13}$ C NMR (101 MHz, $\left.\mathrm{CDCl}_{3}\right): \delta(\mathrm{ppm}) 172.6,172.5,155.4,139.5$ (q, $\left.J=1.5 \mathrm{~Hz}\right), 136.3,135.6,133.0$, $131.0(\mathrm{q}, J=32.5 \mathrm{~Hz}), 129.3,128.7,128.7,128.5,128.3,124.3$ (q, $J=3.7 \mathrm{~Hz}), 124.0$ (q, $J=272.3 \mathrm{~Hz})$, $123.8(\mathrm{q}, J=3.8 \mathrm{~Hz}), 74.9,67.3,62.5,62.2,53.4,16.7,16.5,14.1$.

${ }^{19}$ F NMR (376 MHz, $\left.\mathrm{CDCl}_{3}\right): \delta(\mathrm{ppm})-62.7(3 \mathrm{~F})$.

HRMS (ESI): calculated for $\mathrm{C}_{26} \mathrm{H}_{28} \mathrm{~F}_{3} \mathrm{NO}_{6} \mathrm{Na}[\mathrm{M}+\mathrm{Na}]^{+}: 530.1761$; found: 530.1755 .

( \pm )-1-Benzyl 4-ethyl (2S,3S)-3-hydroxy-2-((E)-2-((E)-1-(methoxyimino)ethyl)-5-(trifluoromethoxy)styryl)-2-methylsuccinate (4ra)

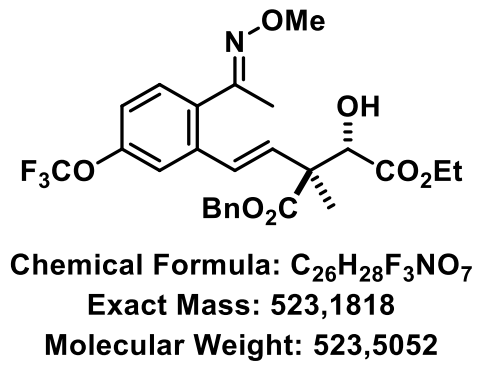

Prepared from (E)-1-(4-(trifluoromethoxy)phenyl)ethan-1-one $O$-methyl oxime $1 \mathbf{r} \quad$ (46.6 mg, $0.20 \mathrm{mmol}, 1.0$ equiv) and benzyl bicyclo[1.1.0]butane-1-carboxylate $2 \mathrm{2a}(75.3 \mathrm{mg}, 0.40 \mathrm{mmol}$, 2.0 equiv) according to the general procedure in the presence of $\left[\mathrm{Cp}^{*} \mathrm{Rh}\left(\mathrm{CH}_{3} \mathrm{CN}\right)_{3}\right]\left(\mathrm{SbF}_{6}\right)_{2}(8.3 \mathrm{mg}$, $0.01 \mathrm{mmol}, 5 \mathrm{~mol} \%$ ). The desired product was obtained after column chromatography (eluent: pentane/EtOAc) and subsequent preparative TLC (eluent: toluene/EtOAc) as a yellow sticky oil (67.9 mg, $0.13 \mathrm{mmol}, 65 \%,>20: 1 \mathrm{dr}, E / Z:>20: 1$ ).

$\mathbf{R}_{\mathbf{f}}($ pentane $/$ EtOAc 80:20) $=0.39$. 
${ }^{1} \mathbf{H}$ NMR $\left(400 \mathrm{MHz}, \mathrm{CDCl}_{3}\right): \delta(\mathrm{ppm}) 7.38-7.27(\mathrm{~m}, 7 \mathrm{H}), 7.14-7.09(\mathrm{~m}, 1 \mathrm{H}), 6.65(\mathrm{~d}, J=16.2 \mathrm{~Hz}$, $1 \mathrm{H}), 6.39(\mathrm{~d}, J=16.2 \mathrm{~Hz}, 1 \mathrm{H}), 5.24-5.14(\mathrm{~m}, 2 \mathrm{H}), 4.63(\mathrm{~s}, 1 \mathrm{H}), 4.24-4.08(\mathrm{~m}, 2 \mathrm{H}), 3.96(\mathrm{~s}, 3 \mathrm{H}), 3.21$ (bs, 1H), 2.10 (s, 3H), $1.42(\mathrm{~s}, 3 \mathrm{H}), 1.20(\mathrm{t}, J=7.1 \mathrm{~Hz}, 3 \mathrm{H})$.

${ }^{13} \mathrm{C}$ NMR (101 MHz, $\left.\mathrm{CDCl}_{3}\right): \delta(\mathrm{ppm}) 172.6,172.6,155.4,149.5$ (q, $\left.J=1.9 \mathrm{~Hz}\right), 137.6,135.6,135.0$, $132.7,130.3,128.8,128.7,128.5,128.3,120.5$ (q, $J=257.7 \mathrm{~Hz}), 120.0,119.3,74.9,67.3,62.5,62.1$, $53.3,16.7,16.7,14.1$.

${ }^{19}$ F NMR (377 MHz, $\left.\mathrm{CDCl}_{3}\right): \delta(\mathrm{ppm})-57.7(3 \mathrm{~F})$.

HRMS (ESI): calculated for $\mathrm{C}_{26} \mathrm{H}_{28} \mathrm{~F}_{3} \mathrm{NO}_{7} \mathrm{Na}[\mathrm{M}+\mathrm{Na}]^{+}:$546.1710; found: 546.1704.

( \pm )-1-Benzyl 4-ethyl (2S,3S)-3-hydroxy-2-((E)-2-((E)-1-(methoxyimino)ethyl)-5-(methylsulfonyl)styryl)-2-methylsuccinate (4sa)<smiles>CCOC(=O)[C@H](O)[C@@](C)(C=Cc1cc(OC)ccc1C(C)=NOC)C(=O)OC</smiles>

Chemical Formula: $\mathrm{C}_{26} \mathrm{H}_{31} \mathrm{NO}_{8} \mathrm{~S}$

Exact Mass: 517,1770

Molecular Weight: 517,5930

Prepared from (E)-1-(4-(methylsulfonyl)phenyl)ethan-1-one $O$-methyl oxime 1s (45.5 mg, $0.20 \mathrm{mmol}$, 1.0 equiv) and benzyl bicyclo[1.1.0]butane-1-carboxylate $2 \mathrm{a}(75.3 \mathrm{mg}, 0.40 \mathrm{mmol}, 2.0$ equiv) according to the general procedure in the presence of $\left[\mathrm{Cp} * \mathrm{Rh}\left(\mathrm{CH}_{3} \mathrm{CN}\right)_{3}\right]\left(\mathrm{SbF}_{6}\right)_{2}(16.7 \mathrm{mg}, 0.02 \mathrm{mmol}, 10 \mathrm{~mol} \%)$. The desired product was obtained after column chromatography (eluent: pentane/EtOAc) and subsequent preparative TLC (eluent: toluene/EtOAc) as a yellow sticky oil ( $80.4 \mathrm{mg}, 0.16 \mathrm{mmol}, 78 \%$, $>20: 1 \mathrm{dr}, E / Z:>20: 1)$.

$\mathbf{R}_{\mathbf{f}}($ pentane $/$ EtOAc 50:50) $=0.38$.

${ }^{1} \mathbf{H}$ NMR $\left(400 \mathrm{MHz}, \mathrm{CDCl}_{3}\right): \delta(\mathrm{ppm}) 8.02(\mathrm{~d}, J=1.9 \mathrm{~Hz}, 1 \mathrm{H}), 7.84-7.77(\mathrm{~m}, 1 \mathrm{H}), 7.46(\mathrm{~d}, J=8.1 \mathrm{~Hz}$, $1 \mathrm{H}), 7.38-7.27(\mathrm{~m}, 5 \mathrm{H}), 6.67(\mathrm{~d}, J=16.2 \mathrm{~Hz}, 1 \mathrm{H}), 6.50(\mathrm{~d}, J=16.2 \mathrm{~Hz}, 1 \mathrm{H}), 5.18(\mathrm{~s}, 2 \mathrm{H}), 4.65(\mathrm{~s}, 1 \mathrm{H})$, $4.25-4.05(\mathrm{~m}, 2 \mathrm{H}), 3.97(\mathrm{~s}, 3 \mathrm{H}), 3.03(\mathrm{~s}, 3 \mathrm{H}), 2.11(\mathrm{~s}, 3 \mathrm{H}), 1.40(\mathrm{~s}, 3 \mathrm{H}), 1.19$ (t, $J=7.1 \mathrm{~Hz}, 3 \mathrm{H})$, one proton signal missing due to broadening.

${ }^{13}$ C NMR (101 MHz, $\left.\mathrm{CDCl}_{3}\right): \delta(\mathrm{ppm}) 172.5,172.3,155.0,141.3,140.8,137.1,135.5,133.9,129.9$, $128.7,128.5,128.4,128.2,126.2,125.8,74.7,67.3,62.5,62.3,53.4,44.6,16.6,16.4,14.1$.

HRMS (ESI): calculated for $\mathrm{C}_{26} \mathrm{H}_{31} \mathrm{NO}_{8} \mathrm{SNa}[\mathrm{M}+\mathrm{Na}]^{+}:$540.1663; found: 540.1660 . 
( \pm )-1-Benzyl 4-ethyl $(2 S, 3 S)-3-h y d r o x y-2-((E)-2-((E)-1-(m e t h o x y i m i n o) e t h y l)-5-(N$-phenylsulfamoyl)styryl)-2-methylsuccinate (4ta)

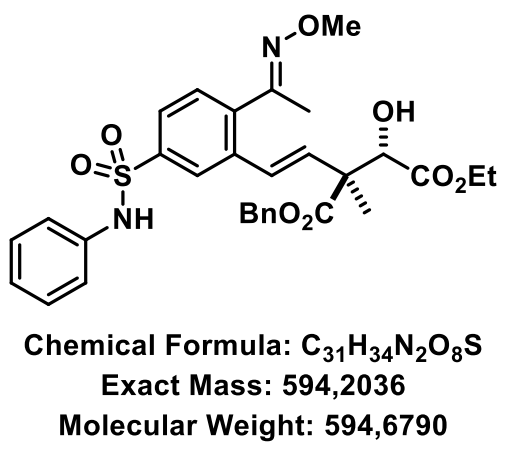

Prepared from (E)-4-(1-(methoxyimino)ethyl)- $N$-phenylbenzenesulfonamide 1t (60.9 mg, $0.20 \mathrm{mmol}$, 1.0 equiv) and benzyl bicyclo[1.1.0]butane-1-carboxylate $\mathbf{2 a}(75.3 \mathrm{mg}, 0.40 \mathrm{mmol}, 2.0$ equiv) according to the general procedure in the presence of $\left[\mathrm{Cp} * \mathrm{Rh}\left(\mathrm{CH}_{3} \mathrm{CN}\right)_{3}\right]\left(\mathrm{SbF}_{6}\right)_{2}(16.7 \mathrm{mg}, 0.02 \mathrm{mmol}, 10 \mathrm{~mol} \%)$. The desired product was obtained after column chromatography (eluent: pentane/EtOAc) as a yellow sticky oil (96.1 mg, $0.16 \mathrm{mmol}, 81 \%,>20: 1 \mathrm{dr}, E / Z:>20: 1)$.

$\mathbf{R}_{\mathbf{f}}($ pentane/EtOAc 65:35) $=0.30$.

${ }^{1} \mathbf{H}$ NMR $\left(400 \mathrm{MHz}, \mathrm{CDCl}_{3}\right): \delta(\mathrm{ppm}) 7.88(\mathrm{~d}, J=2.0 \mathrm{~Hz}, 1 \mathrm{H}), 7.63(\mathrm{dd}, J=8.1,1.9 \mathrm{~Hz}, 1 \mathrm{H}), 7.38-$ $7.28(\mathrm{~m}, 7 \mathrm{H}), 7.22-7.16(\mathrm{~m}, 2 \mathrm{H}), 7.11-7.04(\mathrm{~m}, 3 \mathrm{H}), 6.61(\mathrm{~d}, J=16.2 \mathrm{~Hz}, 1 \mathrm{H}), 6.34(\mathrm{~d}, J=16.2 \mathrm{~Hz}$, $1 \mathrm{H}), 5.17(\mathrm{~s}, 2 \mathrm{H}), 4.64(\mathrm{~d}, J=5.6 \mathrm{~Hz}, 1 \mathrm{H}), 4.22-4.06(\mathrm{~m}, 2 \mathrm{H}), 3.95(\mathrm{~s}, 3 \mathrm{H}), 3.40(\mathrm{~d}, J=5.8 \mathrm{~Hz}, 1 \mathrm{H})$, $2.06(\mathrm{~s}, 3 \mathrm{H}), 1.39(\mathrm{~s}, 3 \mathrm{H}), 1.17(\mathrm{t}, J=7.2 \mathrm{~Hz}, 3 \mathrm{H})$.

${ }^{13}$ C NMR (101 MHz, $\left.\mathrm{CDCl}_{3}\right): \delta(\mathrm{ppm}) 172.5,172.5,155.1,140.4,139.4,136.8,136.4,135.6,133.5$, $129.5,129.4,128.7,128.5,128.4,128.3,126.0,125.6,121.8,74.9,67.3,62.5,62.2,53.4,16.7,16.4$, 14.1, one carbon signal missing due to overlap.

HRMS (ESI): calculated for $\mathrm{C}_{31} \mathrm{H}_{34} \mathrm{~N}_{2} \mathrm{O}_{8} \mathrm{SNa}[\mathrm{M}+\mathrm{Na}]^{+}$: 617.1928; found: 617.1929.

( \pm )-1-Benzyl 4-ethyl (2S,3S)-3-hydroxy-2-((E)-2-((E)-1-(methoxyimino)ethyl)-5-(methylthio)styryl)-2-methylsuccinate (4ua)

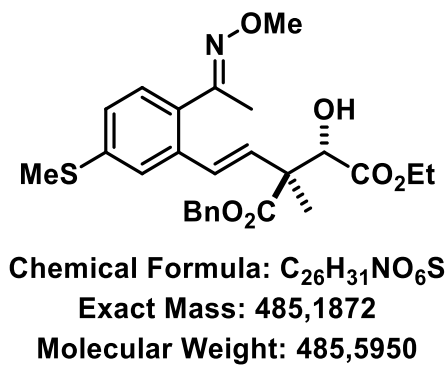

Prepared from (E)-1-(4-(methylthio)phenyl)ethan-1-one $O$-methyl oxime 1u $(39.1 \mathrm{mg}, 0.20 \mathrm{mmol}$, 1.0 equiv) and benzyl bicyclo[1.1.0 ]butane-1-carboxylate $\mathbf{2 a}(75.3 \mathrm{mg}, 0.40 \mathrm{mmol}, 2.0$ equiv) according to the general procedure in the presence of $\left[\mathrm{Cp} * \mathrm{Rh}\left(\mathrm{CH}_{3} \mathrm{CN}\right)_{3}\right]\left(\mathrm{SbF}_{6}\right)_{2}(16.7 \mathrm{mg}, 0.02 \mathrm{mmol}, 10 \mathrm{~mol} \%)$. The desired product was obtained after column chromatography (eluent: pentane/EtOAc) and subsequent preparative TLC (eluent: toluene/EtOAc) as a yellowish sticky oil (42.9 mg, $0.09 \mathrm{mmol}$, $44 \%,>20: 1 \mathrm{dr}, E / Z:>20: 1)$.

$\mathbf{R}_{\mathbf{f}}($ pentane/EtOAc 80:20) $=0.34$. 
${ }^{1} \mathbf{H}$ NMR $\left(400 \mathrm{MHz}, \mathrm{CDCl}_{3}\right): \delta(\mathrm{ppm}) 7.38-7.31(\mathrm{~m}, 6 \mathrm{H}), 7.20(\mathrm{~d}, J=8.1 \mathrm{~Hz}, 1 \mathrm{H}), 7.13(\mathrm{dd}, J=8.1$, $1.9 \mathrm{~Hz}, 1 \mathrm{H}), 6.67(\mathrm{~d}, J=16.2 \mathrm{~Hz}, 1 \mathrm{H}), 6.36(\mathrm{~d}, J=16.2 \mathrm{~Hz}, 1 \mathrm{H}), 5.22-5.14(\mathrm{~m}, 2 \mathrm{H}), 4.64(\mathrm{~d}, J=5.5$ $\mathrm{Hz}, 1 \mathrm{H}), 4.24-4.09$ (m, 2H), 3.95 (s, 3H), 3.20 (d, J=5.4 Hz, 1H), 2.47 (s, 3H), 2.09 (s, 3H), 1.41 (s, $3 \mathrm{H}), 1.21(\mathrm{t}, J=7.1 \mathrm{~Hz}, 3 \mathrm{H})$.

${ }^{13}$ C NMR (101 MHz, $\left.\mathrm{CDCl}_{3}\right): \delta 172.8,172.6,156.0,139.5,136.0,135.7,133.2,131.4,129.8,129.1$, $128.7,128.4,128.3,125.6,124.9,75.0,67.2,62.4,62.0,53.3,16.7,15.9,14.2$, one carbon signal missing due to overlap.

HRMS (ESI): calculated for $\mathrm{C}_{26} \mathrm{H}_{31} \mathrm{NO}_{6} \mathrm{SNa}[\mathrm{M}+\mathrm{Na}]^{+}:$508.1764; found: 508.1761 .

( \pm -1-Benzyl 4-ethyl (2S,3S)-3-hydroxy-2-((E)-2-((E)-1-(methoxyimino)ethyl)-4-methylstyryl)-2methylsuccinate (4va)<smiles>CCOC(=O)[C@H](O)C(C)(C)[C@@H](O)/C=C/c1ccc(C)cc1/C(C)=N/OC</smiles>

Chemical Formula: $\mathrm{C}_{26} \mathrm{H}_{31} \mathrm{NO}_{6}$

Exact Mass: 453,2151

Molecular Weight: 453,5350

Prepared from $(E)-1-(m$-tolyl)ethan-1-one $O$-methyl oxime 1v (32.6 mg, $0.20 \mathrm{mmol}, 1.0$ equiv) and benzyl bicyclo[1.1.0]butane-1-carboxylate $2 \mathrm{a}(75.3 \mathrm{mg}, 0.40 \mathrm{mmol}, 2.0$ equiv) according to the general procedure in the presence of $\left[\mathrm{Cp}^{*} \mathrm{Rh}\left(\mathrm{CH}_{3} \mathrm{CN}\right)_{3}\right]\left(\mathrm{SbF}_{6}\right)_{2}(8.3 \mathrm{mg}, 0.01 \mathrm{mmol}, 5 \mathrm{~mol} \%)$. The desired product was obtained after column chromatography (eluent: pentane/EtOAc) and subsequent preparative TLC (eluent: toluene/EtOAc) as a yellowish sticky oil (58.4 mg, $0.13 \mathrm{mmol}, 64 \%,>20: 1 \mathrm{dr}$, $>20: 1$ site selectivity, $E / Z:>20: 1)$.

$\mathbf{R}_{\mathbf{f}}($ pentane $/$ EtOAc 80:20) $=0.32$.

${ }^{1}$ H NMR $\left(400 \mathrm{MHz}, \mathrm{CDCl}_{3}\right): \delta(\mathrm{ppm}) 7.41-7.28(\mathrm{~m}, 6 \mathrm{H}), 7.11(\mathrm{~d}, J=7.8 \mathrm{~Hz}, 1 \mathrm{H}), 7.07(\mathrm{~s}, 1 \mathrm{H}), 6.64$ $(\mathrm{d}, J=16.2 \mathrm{~Hz}, 1 \mathrm{H}), 6.34(\mathrm{~d}, J=16.2 \mathrm{~Hz}, 1 \mathrm{H}), 5.18(\mathrm{~s}, 2 \mathrm{H}), 4.64(\mathrm{~d}, J=5.5 \mathrm{~Hz}, 1 \mathrm{H}), 4.24-4.08(\mathrm{~m}$, 2H), $3.96(\mathrm{~s}, 3 \mathrm{H}), 3.20(\mathrm{~d}, J=5.5 \mathrm{~Hz}, 1 \mathrm{H}), 2.33(\mathrm{~s}, 3 \mathrm{H}), 2.10(\mathrm{~s}, 3 \mathrm{H}), 1.41(\mathrm{~s}, 3 \mathrm{H}), 1.20(\mathrm{t}, J=7.1 \mathrm{~Hz}$, $3 \mathrm{H})$.

${ }^{13} \mathrm{C}$ NMR (101 MHz, $\left.\mathrm{CDCl}_{3}\right): \delta$ (ppm) 173.0, 172.7, 156.8, 137.7, 136.3, 135.8, 132.6, 130.1, 129.7, $129.2,128.6,128.4,128.3,126.8,75.1,67.1,62.3,61.9,53.3,21.2,16.9,16.7,14.2$, one carbon signal missing due to overlap.

HRMS (ESI): calculated for $\mathrm{C}_{26} \mathrm{H}_{31} \mathrm{NO}_{6} \mathrm{Na}[\mathrm{M}+\mathrm{Na}]^{+}$: 476.2044; found: 476.2037. 


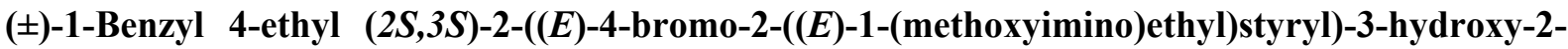
methylsuccinate (4wa)<smiles>CCOC(=O)[C@H](O)[C@@](C)(/C=C/c1ccc(Br)cc1/C(C)=N/OC)C(=O)O</smiles>

Chemical Formula: $\mathrm{C}_{25} \mathrm{H}_{28} \mathrm{BrNO}_{6}$

Exact Mass: 517,1100

Molecular Weight: $\mathbf{5 1 8 , 4 0 4 0}$

Prepared from (E)-1-(3-bromophenyl)ethan-1-one $O$-methyl oxime 1x (45.6 mg, $0.20 \mathrm{mmol}, 1.0$ equiv) and benzyl bicyclo[1.1.0]butane-1-carboxylate 2a (75.3 $\mathrm{mg}, 0.40 \mathrm{mmol}, 2.0$ equiv) according to the general procedure in the presence of $\left[\mathrm{Cp}^{*} \mathrm{Rh}\left(\mathrm{CH}_{3} \mathrm{CN}\right)_{3}\right]\left(\mathrm{SbF}_{6}\right)_{2}(8.3 \mathrm{mg}, 0.01 \mathrm{mmol}, 5 \mathrm{~mol} \%)$. The desired product was obtained after column chromatography (eluent: pentane/EtOAc) and subsequent preparative TLC (eluent: toluene/EtOAc) as a yellowish sticky oil ( $65.3 \mathrm{mg}, 0.12 \mathrm{mmol}, 63 \%,>20: 1 \mathrm{dr}$, $>20: 1$ site selectivity, $E / Z:>20: 1)$.

$\mathbf{R}_{\mathbf{f}}($ pentane $/$ EtOAc 80:20) $=0.32$.

${ }^{1}$ H NMR $\left(400 \mathrm{MHz}, \mathrm{CDCl}_{3}\right): \delta(\mathrm{ppm}) 7.44-7.39(\mathrm{~m}, 2 \mathrm{H}), 7.36-7.27(\mathrm{~m}, 6 \mathrm{H}), 6.59(\mathrm{~d}, J=16.2 \mathrm{~Hz}$, $1 \mathrm{H}), 6.37(\mathrm{~d}, J=16.2 \mathrm{~Hz}, 1 \mathrm{H}), 5.21-5.13(\mathrm{~m}, 2 \mathrm{H}), 4.63(\mathrm{~d}, J=5.4 \mathrm{~Hz}, 1 \mathrm{H}), 4.24-4.08(\mathrm{~m}, 2 \mathrm{H}), 3.96$ (s, 3H), 3.22 (d, $J=5.5 \mathrm{~Hz}, 1 \mathrm{H}), 2.08(\mathrm{~s}, 3 \mathrm{H}), 1.40(\mathrm{~s}, 3 \mathrm{H}), 1.20(\mathrm{t}, J=7.1 \mathrm{~Hz}, 3 \mathrm{H})$.

${ }^{13} \mathrm{C}$ NMR (101 MHz, $\left.\mathrm{CDCl}_{3}\right): \delta$ (ppm) 172.7, 172.6, 155.3, 138.0, 135.7, 134.5, 131.8, 131.8, 131.5, $128.8,128.7,128.5,128.4,128.3,121.5,74.9,67.2,62.4,62.1,53.3,16.7,16.6,14.2$.

HRMS (ESI): calculated for $\mathrm{C}_{25} \mathrm{H}_{28} \mathrm{BrNO}_{6} \mathrm{Na}[\mathrm{M}+\mathrm{Na}]^{+}:$542.0975; found: 542.0972 .

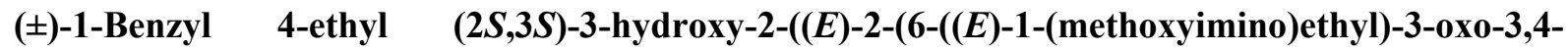
dihydro-2 $H$-benzo $[b][1,4]$ oxazin-7-yl)vinyl)-2-methylsuccinate (4xa)<smiles>CCOC(=O)[C@H](O)[C@@](C)(/C=C/c1cc2c(cc1/C(C)=N/OC)NC(=O)CO2)C(=O)O</smiles>

Chemical Formula: $\mathrm{C}_{27} \mathrm{H}_{30} \mathrm{~N}_{2} \mathrm{O}_{8}$

Exact Mass: 510,2002

Molecular Weight: $\mathbf{5 1 0 , 5 4 3 0}$

Prepared from (E)-6-(1-(methoxyimino)ethyl)-2H-benzo[b][1,4]oxazin-3(4H)-one $\mathbf{1 x} \quad$ (44.0 mg, $0.20 \mathrm{mmol}, 1.0$ equiv) and benzyl bicyclo[1.1.0]butane-1-carboxylate $\mathbf{2 a}(75.3 \mathrm{mg}, 0.40 \mathrm{mmol}$, 2.0 equiv) according to the general procedure in the presence of $\left[\mathrm{Cp}^{*} \mathrm{Rh}\left(\mathrm{CH}_{3} \mathrm{CN}\right)_{3}\right]\left(\mathrm{SbF}_{6}\right)_{2}(16.7 \mathrm{mg}$, $0.02 \mathrm{mmol}, 10 \mathrm{~mol} \%$ ). The desired product was obtained after column chromatography (eluent: pentane/EtOAc) and subsequent preparative TLC (eluent: toluene/EtOAc) as an orange sticky oil (74.0 mg, $0.14 \mathrm{mmol}, 72 \%,>20: 1 \mathrm{dr},>20: 1$ site selectivity, $E / Z:>20: 1$ ).

$\mathbf{R}_{\mathbf{f}}($ pentane/EtOAc 40:60) $=0.42$.

${ }^{1}$ H NMR (400 MHz, CDCl $)$ ): $\delta$ (ppm) 9.29 (bs, 1H), $7.40-7.30(\mathrm{~m}, 5 \mathrm{H}), 7.05$ (s, 1H), $6.69(\mathrm{~s}, 1 \mathrm{H})$, $6.53(\mathrm{~d}, J=16.1 \mathrm{~Hz}, 1 \mathrm{H}), 6.32(\mathrm{~d}, J=16.1 \mathrm{~Hz}, 1 \mathrm{H}), 5.17(\mathrm{~s}, 2 \mathrm{H}), 4.66(\mathrm{~s}, 1 \mathrm{H}), 4.60(\mathrm{~s}, 2 \mathrm{H}), 4.24-4.08$ (m, 2H), 3.95 (s, 3H), $3.22(\mathrm{bs}, 1 \mathrm{H}), 2.06(\mathrm{~s}, 3 \mathrm{H}), 1.40(\mathrm{~s}, 3 \mathrm{H}), 1.21(\mathrm{t}, J=7.1 \mathrm{~Hz}, 3 \mathrm{H})$. 
${ }^{13}$ C NMR (101 MHz, $\left.\mathrm{CDCl}_{3}\right): \delta$ (ppm) 172.8, 172.7, 166.0, 155.7, 143.9, 135.7, 131.9, 131.1, 130.7, $128.7,128.4,128.3,125.4,116.3,114.6,75.2,67.2,62.3,62.0,53.3,16.9,16.6,14.2$, two carbon signals missing due to overlap.

HRMS (ESI): calculated for $\mathrm{C}_{27} \mathrm{H}_{30} \mathrm{~N}_{2} \mathrm{O}_{8} \mathrm{Na}[\mathrm{M}+\mathrm{Na}]^{+}:$533.1894; found: 533.1889 .

( \pm -1-Benzyl 4-ethyl (2S,3S)-3-hydroxy-2-((E)-2-((E)-1-(methoxyimino)ethyl)-4,5-dimethylstyryl)2-methylsuccinate (4ya)<smiles>CCOC(O)[C@H](O)[C@](C)(C=Cc1cc(C)c(C)cc1C(C)=O)C(=O)O</smiles>

Chemical Formula: $\mathrm{C}_{27} \mathrm{H}_{33} \mathrm{NO}_{6}$

Exact Mass: 467,2308

Molecular Weight: 467,5620

Prepared from (E)-1-(3,4-dimethylphenyl)ethan-1-one $O$-methyl oxime 1y $(35.4 \mathrm{mg}, 0.20 \mathrm{mmol}$, 1.0 equiv) and benzyl bicyclo[1.1.0]butane-1-carboxylate $\mathbf{2 a}(75.3 \mathrm{mg}, 0.40 \mathrm{mmol}, 2.0$ equiv) according to the general procedure in the presence of $\left[\mathrm{Cp} * \mathrm{Rh}\left(\mathrm{CH}_{3} \mathrm{CN}\right)_{3}\right]\left(\mathrm{SbF}_{6}\right)_{2}(16.7 \mathrm{mg}, 0.02 \mathrm{mmol}, 10 \mathrm{~mol} \%)$. The desired product was obtained after column chromatography (eluent: pentane/EtOAc) and subsequent preparative TLC (eluent: toluene/EtOAc) as a yellowish sticky oil (58.3 mg, $0.12 \mathrm{mmol}$, $62 \%,>20: 1 \mathrm{dr},>20: 1$ site selectivity, $E / Z:>20: 1)$.

$\mathbf{R}_{\mathbf{f}}($ pentane $/$ EtOAc $70: 30)=0.75$.

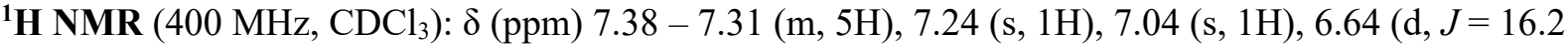
$\mathrm{Hz}, 1 \mathrm{H}), 6.33(\mathrm{~d}, J=16.2 \mathrm{~Hz}, 1 \mathrm{H}), 5.18(\mathrm{~d}, J=1.8 \mathrm{~Hz}, 2 \mathrm{H}), 4.65(\mathrm{~d}, J=5.0 \mathrm{~Hz}, 1 \mathrm{H}), 4.28-4.06(\mathrm{~m}$, $2 \mathrm{H}), 3.96(\mathrm{~s}, 3 \mathrm{H}), 3.21(\mathrm{~d}, J=5.4 \mathrm{~Hz}, 1 \mathrm{H}), 2.25(\mathrm{~m}, 6 \mathrm{H}), 2.09(\mathrm{~s}, 3 \mathrm{H}), 1.42(\mathrm{~s}, 3 \mathrm{H}), 1.21(\mathrm{t}, J=7.1 \mathrm{~Hz}$, $3 \mathrm{H})$.

${ }^{13} \mathbf{C}$ NMR (101 MHz, $\left.\mathrm{CDCl}_{3}\right): \delta(\mathrm{ppm}) 173.0,172.7,156.7,137.4,136.4,135.8,133.9,132.8,129.8$, $129.8,129.7,128.6,128.3,128.3,128.0,75.1,67.1,62.3,61.9,53.3,19.6,19.5,16.9,16.7,14.2$.

HRMS (ESI): calculated for $\mathrm{C}_{27} \mathrm{H}_{33} \mathrm{NO}_{6} \mathrm{Na}[\mathrm{M}+\mathrm{Na}]^{+}$: 490.2200; found: 490.2197 .

( $($ )-1-Benzyl 4-ethyl $(2 S, 3 S)$-3-hydroxy-2-((E)-2-((E)-8-(methoxyimino)-5,6,7,8-tetrahydronaphthalen-1-yl)vinyl)-2-methylsuccinate (4za)

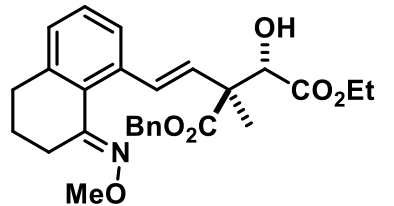

Chemical Formula: $\mathrm{C}_{27} \mathrm{H}_{31} \mathrm{NO}_{6}$

Exact Mass: 465,2151

Molecular Weight: 465,5460

Prepared from (E)-3,4-dihydronaphthalen-1(2H)-one $O$-methyl oxime $\mathbf{1 z}(35.0 \mathrm{mg}, 0.20 \mathrm{mmol}$, 1.0 equiv) and benzyl bicyclo[1.1.0]butane-1-carboxylate $\mathbf{2 a}(75.3 \mathrm{mg}, 0.40 \mathrm{mmol}, 2.0$ equiv) according to the general procedure in the presence of $\left[\mathrm{Cp}^{*} \mathrm{Rh}\left(\mathrm{CH}_{3} \mathrm{CN}\right)_{3}\right]\left(\mathrm{SbF}_{6}\right)_{2}(16.7 \mathrm{mg}, 0.02 \mathrm{mmol}, 10 \mathrm{~mol} \%)$. The desired product was obtained after column chromatography (eluent: pentane/EtOAc) and 
subsequent preparative TLC (eluent: toluene/EtOAc) as a yellowish sticky oil $(53.7 \mathrm{mg}, 0.12 \mathrm{mmol}$, $58 \%,>20: 1 \mathrm{dr}, E / Z:>20: 1)$.

$\mathbf{R}_{\mathbf{f}}($ pentane/EtOAc 80:20) $=0.35$.

${ }^{1} \mathbf{H}$ NMR $\left(400 \mathrm{MHz}, \mathrm{CDCl}_{3}\right): \delta(\mathrm{ppm}) 7.41-7.29(\mathrm{~m}, 6 \mathrm{H}), 7.25-7.17(\mathrm{~m}, 2 \mathrm{H}), 7.07(\mathrm{~d}, J=7.4 \mathrm{~Hz}$, $1 \mathrm{H}), 6.23(\mathrm{~d}, J=16.1 \mathrm{~Hz}, 1 \mathrm{H}), 5.26-5.13(\mathrm{~m}, 2 \mathrm{H}), 4.68(\mathrm{~d}, J=5.1 \mathrm{~Hz}, 1 \mathrm{H}), 4.26-4.08(\mathrm{~m}, 2 \mathrm{H}), 3.97$ $(\mathrm{s}, 3 \mathrm{H}), 3.19(\mathrm{~d}, J=5.2 \mathrm{~Hz}, 1 \mathrm{H}), 2.75(\mathrm{t}, J=6.8 \mathrm{~Hz}, 2 \mathrm{H}), 2.69-2.62(\mathrm{~m}, 2 \mathrm{H}), 1.83-1.71(\mathrm{~m}, 2 \mathrm{H}), 1.47$ (s, 3H), $1.21(\mathrm{t}, J=7.1 \mathrm{~Hz}, 3 \mathrm{H})$.

${ }^{13} \mathrm{C}$ NMR (101 MHz, $\left.\mathrm{CDCl}_{3}\right): \delta$ (ppm) 173.1, 172.5, 155.0, 141.8, 136.4, 135.9, 134.0, 128.9, 128.6, $128.4,128.4,128.3,128.2,127.7,126.5,75.2,67.0,62.3,62.1,53.4,31.0,25.6,21.2,16.4,14.2$.

HRMS (ESI): calculated for $\mathrm{C}_{27} \mathrm{H}_{31} \mathrm{NO}_{6} \mathrm{Na}[\mathrm{M}+\mathrm{Na}]^{+}: 488.2044$; found: 488.2037 .

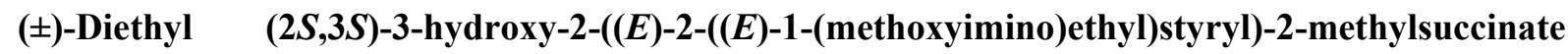
(4ab)<smiles>CCOC(=O)[C@H](O)[C@@](C)(/C=C/c1ccccc1/C(C)=N/OC)C(=O)OCC</smiles>

Chemical Formula: $\mathrm{C}_{20} \mathrm{H}_{27} \mathrm{NO}_{6}$

Exact Mass: 377,1838

Molecular Weight: $\mathbf{3 7 7 , 4 3 7 0}$

Prepared from (E)-1-phenylethan-1-one $O$-methyl oxime 1a (29.8 mg, $0.20 \mathrm{mmol}, 1.0$ equiv) and ethyl bicyclo[1.1.0]butane-1-carboxylate $\mathbf{2 b}(50.5 \mathrm{mg}, 0.40 \mathrm{mmol}, 2.0$ equiv) according to the general procedure in the presence of $\left[\mathrm{Cp}^{*} \mathrm{Rh}\left(\mathrm{CH}_{3} \mathrm{CN}\right)_{3}\right]\left(\mathrm{SbF}_{6}\right)_{2}(8.3 \mathrm{mg}, 0.01 \mathrm{mmol}, 5 \mathrm{~mol} \%)$. The desired product was obtained after column chromatography (eluent: pentane/EtOAc) and subsequent preparative TLC (eluent: toluene/EtOAc) as a yellowish sticky oil (54.0 mg, $0.14 \mathrm{mmol}, 72 \%,>20: 1 \mathrm{dr}$, $E / Z:>20: 1)$.

$\mathbf{R}_{\mathbf{f}}($ pentane $/$ EtOAc 80:20) $=0.28$.

${ }^{1} \mathbf{H}$ NMR $\left(400 \mathrm{MHz}, \mathrm{CDCl}_{3}\right): \delta(\mathrm{ppm}) 7.49(\mathrm{~d}, J=7.6 \mathrm{~Hz}, 1 \mathrm{H}), 7.34-7.21(\mathrm{~m}, 3 \mathrm{H}), 6.66(\mathrm{~d}, J=16.2$ $\mathrm{Hz}, 1 \mathrm{H}), 6.36(\mathrm{~d}, J=16.2 \mathrm{~Hz}, 1 \mathrm{H}), 4.58(\mathrm{~s}, 1 \mathrm{H}), 4.29-4.15(\mathrm{~m}, 4 \mathrm{H}), 3.97(\mathrm{~s}, 3 \mathrm{H}), 3.10(\mathrm{bs}, 1 \mathrm{H}), 2.13$ (s, 3H), $1.39(\mathrm{~s}, 3 \mathrm{H}), 1.31-1.23(\mathrm{~m}, 6 \mathrm{H})$.

${ }^{13} \mathrm{C}$ NMR (101 MHz, $\left.\mathrm{CDCl}_{3}\right): \delta$ (ppm) 173.1, 172.7, 156.6, 136.4, 135.5, 131.3, 129.5, 128.9, 128.6, $127.8,126.8,75.2,62.3,62.0,61.4,53.1,16.9,16.8,14.2,14.2$.

HRMS (ESI): calculated for $\mathrm{C}_{20} \mathrm{H}_{27} \mathrm{NO}_{6} \mathrm{Na}[\mathrm{M}+\mathrm{Na}]^{+}: 400.1731$; found: 400.1727 . 

succinate (4ac)

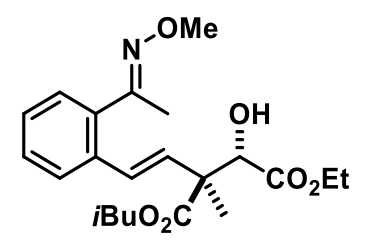

Chemical Formula: $\mathrm{C}_{22} \mathrm{H}_{31} \mathrm{NO}_{6}$

Exact Mass: 405,2151

Molecular Weight: 405,4910

Prepared from (E)-1-phenylethan-1-one $O$-methyl oxime 1a $(29.8 \mathrm{mg}, 0.20 \mathrm{mmol}, 1.0$ equiv) and isobutyl bicyclo[1.1.0]butane-1-carboxylate $2 \mathrm{c}(61.7 \mathrm{mg}, 0.40 \mathrm{mmol}, 2.0$ equiv) according to the general procedure in the presence of $\left[\mathrm{Cp}^{*} \mathrm{Rh}\left(\mathrm{CH}_{3} \mathrm{CN}\right)_{3}\right]\left(\mathrm{SbF}_{6}\right)_{2}(8.3 \mathrm{mg}, 0.01 \mathrm{mmol}, 5 \mathrm{~mol} \%)$. The desired product was obtained after column chromatography (eluent: pentane/EtOAc) and subsequent preparative TLC (eluent: toluene/EtOAc) as a yellowish sticky oil (56.1 mg, $0.14 \mathrm{mmol}, 69 \%,>20: 1 \mathrm{dr}$, $E / Z:>20: 1)$.

$\mathbf{R}_{\mathbf{f}}($ pentane $/$ EtOAc 80:20) $=0.33$.

${ }^{1}$ H NMR $\left(400 \mathrm{MHz}, \mathrm{CDCl}_{3}\right): \delta(\mathrm{ppm}) 7.49(\mathrm{~d}, J=7.4 \mathrm{~Hz}, 1 \mathrm{H}), 7.33-7.24(\mathrm{~m}, 3 \mathrm{H}), 6.68(\mathrm{~d}, J=16.2$ $\mathrm{Hz}, 1 \mathrm{H}), 6.38(\mathrm{~d}, J=16.2 \mathrm{~Hz}, 1 \mathrm{H}), 4.60(\mathrm{~d}, J=5.6 \mathrm{~Hz}, 1 \mathrm{H}), 4.28-4.18(\mathrm{~m}, 2 \mathrm{H}), 3.97(\mathrm{~s}, 3 \mathrm{H}), 3.95-$ $3.88(\mathrm{~m}, 2 \mathrm{H}), 3.24(\mathrm{~d}, J=5.6 \mathrm{~Hz}, 1 \mathrm{H}), 2.13(\mathrm{~s}, 3 \mathrm{H}), 2.04-1.90(\mathrm{~m}, J=6.7 \mathrm{~Hz}, 1 \mathrm{H}), 1.40(\mathrm{~s}, 3 \mathrm{H}), 1.26$ (t, $J=7.1 \mathrm{~Hz}, 3 \mathrm{H}), 0.94(\mathrm{~d}, J=6.7 \mathrm{~Hz}, 6 \mathrm{H})$.

${ }^{13} \mathrm{C}$ NMR (101 MHz, $\left.\mathrm{CDCl}_{3}\right): \delta(\mathrm{ppm}) 173.1,172.7,156.6,136.4,135.5,131.2,129.6,128.9,128.6$, $127.7,126.8,75.1,71.5,62.3,61.9,53.3,27.8,19.2,19.2,16.8,16.8,14.2$.

HRMS (ESI): calculated for $\mathrm{C}_{22} \mathrm{H}_{31} \mathrm{NO}_{6} \mathrm{Na}[\mathrm{M}+\mathrm{Na}]^{+}: 428.2044$; found: 428.2034 .

( \pm )-Ethyl (2S,3S,E)-2-hydroxy-3-(methoxy(methyl)carbamoyl)-5-(2-((E)-1-(methoxyimino)ethyl)phenyl)-3-methylpent-4-enoate (4ad)

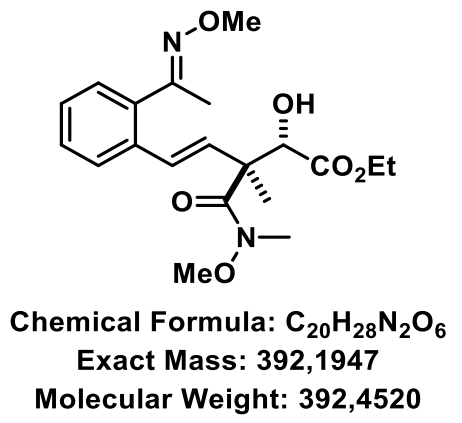

Prepared from $(E)$-1-phenylethan-1-one $O$-methyl oxime 1a $(29.8 \mathrm{mg}, 0.20 \mathrm{mmol}, 1.0$ equiv) and $\mathrm{N}$ methoxy- $N$-methylbicyclo[1.1.0]butane-1-carboxamide $2 \mathrm{a}(56.5 \mathrm{mg}, 0.40 \mathrm{mmol}, 2.0$ equiv) according to the general procedure in the presence of $\left[\mathrm{Cp} * \mathrm{Rh}\left(\mathrm{CH}_{3} \mathrm{CN}\right)_{3}\right]\left(\mathrm{SbF}_{6}\right)_{2}(16.7 \mathrm{mg}, 0.02 \mathrm{mmol}, 10 \mathrm{~mol} \%)$. The desired product was obtained after column chromatography (eluent: pentane/EtOAc) and subsequent preparative TLC (eluent: toluene/EtOAc) as a yellowish sticky oil ( $40.2 \mathrm{mg}, 0.10 \mathrm{mmol}$, $51 \%,>20: 1 \mathrm{dr}, E / Z:>20: 1)$.

$\mathbf{R}_{\mathbf{f}}($ pentane $/$ EtOAc 60:40) $=0.29$. 
${ }^{1} \mathbf{H}$ NMR $\left(400 \mathrm{MHz}, \mathrm{CDCl}_{3}\right): \delta(\mathrm{ppm}) 7.50(\mathrm{~d}, J=7.5 \mathrm{~Hz}, 1 \mathrm{H}), 7.29-7.13(\mathrm{~m}, 3 \mathrm{H}), 6.64-6.49(\mathrm{~m}$, $2 \mathrm{H}), 4.67(\mathrm{~d}, J=7.2 \mathrm{~Hz}, 1 \mathrm{H}), 4.14(\mathrm{q}, J=7.1 \mathrm{~Hz}, 2 \mathrm{H}), 3.89(\mathrm{~s}, 3 \mathrm{H}), 3.50-3.43(\mathrm{~m}, 4 \mathrm{H}), 3.12(\mathrm{~s}, 3 \mathrm{H})$, $2.09(\mathrm{~s}, 3 \mathrm{H}), 1.40(\mathrm{~s}, 3 \mathrm{H}), 1.19(\mathrm{t}, J=7.1 \mathrm{~Hz}, 3 \mathrm{H})$.

${ }^{13}$ C NMR (101 MHz, $\left.\mathrm{CDCl}_{3}\right): \delta$ (ppm) 174.8, 172.7, 156.4, 136.4, 135.6, 132.1, 128.9, 128.8, 128.0, $127.6,126.1,75.3,62.0,62.0,61.1,53.3,33.9,18.5,16.8,14.3$.

HRMS (ESI): calculated for $\mathrm{C}_{20} \mathrm{H}_{28} \mathrm{~N}_{2} \mathrm{O}_{6} \mathrm{Na}[\mathrm{M}+\mathrm{Na}]^{+}$: 415.1840; found: 415.1832 .

\subsection{Scale-up Experiment}

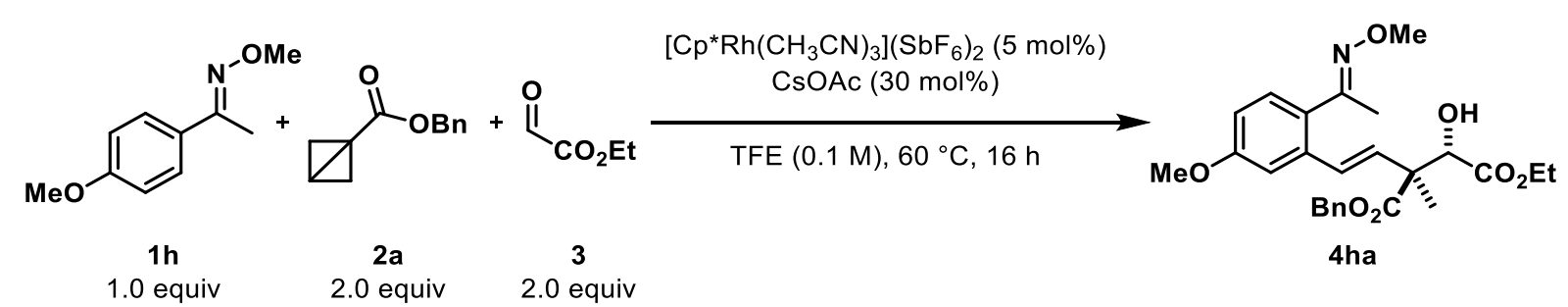

An oven-dried $50 \mathrm{~mL}$ Schlenk tube equipped with a stir bar was charged with CsOAc $(57.6 \mathrm{mg}$, $0.30 \mathrm{mmol}, 0.3$ equiv) in a glovebox. $\left[\mathrm{Cp} * \mathrm{Rh}\left(\mathrm{CH}_{3} \mathrm{CN}\right)_{3}\right]\left(\mathrm{SbF}_{6}\right)_{2}(41.6 \mathrm{mg}, 0.05 \mathrm{mmol}, 5 \mathrm{~mol} \%)$, TFE $(10 \mathrm{~mL}, 0.1 \mathrm{M}),(E)-1-(4-m e t h o x y p h e n y l) e t h a n-1-o n e ~ O$-methyl oxime $\mathbf{1 h}$ (179 $\mathrm{mg}, 1.00 \mathrm{mmol}, 1.0$ equiv), benzyl bicyclo[1.1.0]butane-1-carboxylate 2a (377 $\mathrm{mg}, 2.00 \mathrm{mmol}, 2.0$ equiv) and ethyl glyoxylate 3 ( $407 \mu \mathrm{L}$ of a $50 \mathrm{wt} \%$ solution in toluene, $2.00 \mathrm{mmol}, 2.0$ equiv) were added sequentially under a positive stream of argon. The tube was sealed and the reaction was stirred for $16 \mathrm{~h}$ at $60{ }^{\circ} \mathrm{C}$. The reaction was cooled to room temperature and an aliquot $(5 \mu \mathrm{L})$ was taken, filtered through a plug of Celite and analyzed by LC-UV. The reaction mixture was then concentrated and purified by column chromatography (eluent: pentane/EtOAc) to deliver the desired product as a yellowish sticky oil (350 mg, $0.75 \mathrm{mmol}, 75 \%,>20: 1 \mathrm{dr}, E / Z:>20: 1$ ).

For characterization data see section 3.2.

\subsection{Limitation Studies}

a) Directing groups<smiles>O=Cc1ccccc1</smiles><smiles>CC(=O)c1ccccc1</smiles>

n.r.<smiles>COC(=O)c1ccccc1</smiles>

n.r.<smiles>CON(C)C(=O)c1ccccc1</smiles>

n.r.

b) Strained coupling partners<smiles>CC(C)CC=O</smiles>

traces<smiles>C1=CC2C=C1C2</smiles>

no incorporation<smiles>O=Cc1ccccc1</smiles>

traces<smiles>O=Cc1ccc([N+](=O)[O-])cc1</smiles>

$5 \%$

Figure S1. Unsuccessful directing groups and coupling partners. 
A summary of directing groups, strained cyclic coupling partners and aldehydes which showed low or no reactivity is presented in Figure S1. We investigated carbonyl-containing directing groups and found that benzaldehyde, acetophenone, methyl benzoate as well as $N$-methoxy- $N$-methylbenzamide were unreactive under the developed reaction conditions. Potent further strained molecules such as 1azabicyclo[1.1.0] butane $^{8}$ and [1.1.1]propellane ${ }^{9}$ were not incorporated in the product structure. Furthermore, several aldehydes were examined. However, low reactivity was observed for isovaleraldehyde, benzaldehyde and 4-nitrobenzaldehyde. 


\section{Syntheses of $\beta$-Lactones}

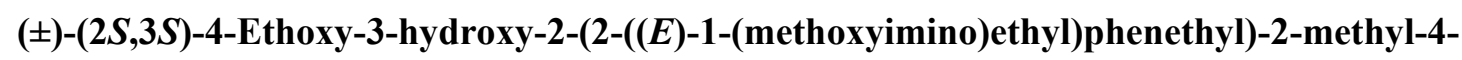
oxobutanoic acid (5)
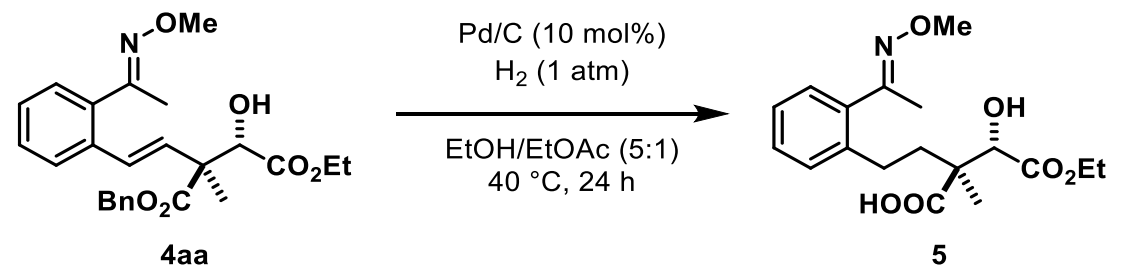

Based on a modified procedure from Proudfoot ${ }^{10}$ an oven-dried $10 \mathrm{~mL}$ Schlenk tube equipped with a stir bar was charged with $\mathrm{Pd} / \mathrm{C}(10 \mathrm{wt} \%, 14.5 \mathrm{mg}, 0.014 \mathrm{mmol}, 10 \mathrm{~mol} \%)$. Compound 4aa $(59.9 \mathrm{mg}$, $0.14 \mathrm{mmol}, 1.0$ equiv) was added as a solution in $\mathrm{EtOH} / \mathrm{EtOAc}(5: 1,0.68 \mathrm{~mL}, 0.2 \mathrm{M})$ and the reaction vessel was purged with $\mathrm{H}_{2}$. The reaction was then stirred at $40{ }^{\circ} \mathrm{C}$ for $24 \mathrm{~h}$ under a $\mathrm{H}_{2}$ atmosphere. After cooling to room temperature the mixture was filtered through a plug of Celite and purification by preparative TLC (eluent: DCM/EtOH) yielded the desired product as a yellowish sticky oil (36.6 mg, $0.10 \mathrm{mmol}, 77 \%)$.

$\mathbf{R}_{\mathbf{f}}(\mathrm{DCM} / \mathrm{EtOH} 90: 10)=0.60$.

${ }^{1} \mathbf{H}$ NMR $\left(400 \mathrm{MHz}, \mathrm{CDCl}_{3}\right): \delta(\mathrm{ppm}) 7.25-7.16(\mathrm{~m}, 4 \mathrm{H}), 4.47$ (bs, 1H), $4.34-4.15(\mathrm{~m}, 2 \mathrm{H}), 3.95$ (s, $3 \mathrm{H}), 2.85-2.73(\mathrm{~m}, 1 \mathrm{H}), 2.68-2.57(\mathrm{~m}, 1 \mathrm{H}), 2.16(\mathrm{~s}, 3 \mathrm{H}), 2.12-2.03(\mathrm{~m}, 1 \mathrm{H}), 1.99-1.90(\mathrm{~m}, 1 \mathrm{H})$, $1.29-1.22(\mathrm{~m}, 3 \mathrm{H}), 1.20(\mathrm{~s}, 3 \mathrm{H})$, two proton signals missing due to broadening.

${ }^{13}$ C NMR (101 MHz, $\left.\mathrm{CDCl}_{3}\right): \delta$ (ppm) 180.6, 173.5, 156.5, 139.7, 137.3, 130.1, 128.7, 128.6, 126.3, $77.4,74.8,62.4,61.8,50.5,38.4,28.3,16.9,14.1$.

HRMS (ESI): calculated for $\mathrm{C}_{18} \mathrm{H}_{25} \mathrm{NO}_{6} \mathrm{Na}[\mathrm{M}+\mathrm{Na}]^{+}: 374.1574$; found: 374.1572 .

( \pm )-Ethyl (2S,3S)-3-(2-((E)-1-(methoxyimino)ethyl)phenethyl)-3-methyl-4-oxooxetane-2-carboxylate (6)
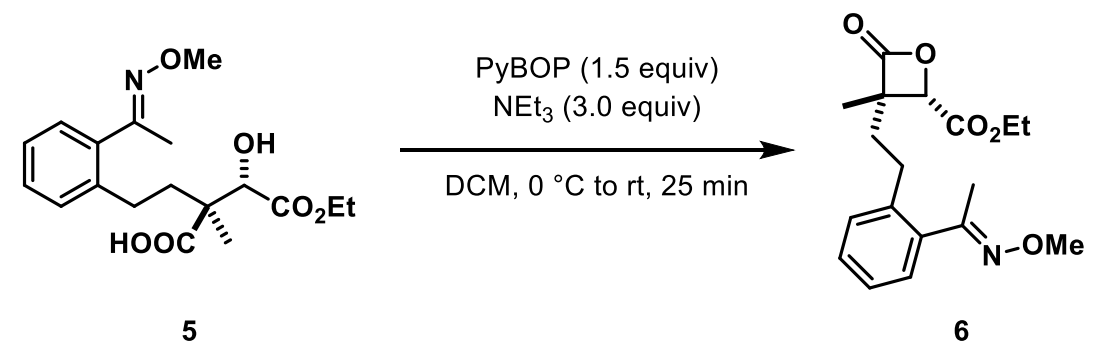

According to a literature procedure from Shuto ${ }^{11}$ compound $5(38.2 \mathrm{mg}, 0.11 \mathrm{mmol}, 1.0$ equiv) was dissolved in DCM $(10.9 \mathrm{~mL}, 0.01 \mathrm{M})$ and cooled to $0{ }^{\circ} \mathrm{C}$. $\mathrm{NEt}_{3}(45.5 \mu \mathrm{L}, 0.33 \mathrm{mmol}, 3.0$ equiv) and PyBOP $\left(84.9 \mathrm{mg}, 0.16 \mathrm{mmol}, 1.5\right.$ equiv) were added and the reaction was stirred at $0{ }^{\circ} \mathrm{C}$ for another 5 min before being warmed to room temperature. After $25 \mathrm{~min}$ the mixture was diluted with EtOAc and washed with $\mathrm{HCl}(1 \mathrm{M})$, sat. aqueous $\mathrm{NaHCO}_{3}$ and brine. The organic layer was dried over $\mathrm{Na}_{2} \mathrm{SO}_{4}$ and purification by column chromatography (eluent: pentane/EtOAc) yielded the desired product as a pale yellow oil (27.8 mg, $0.08 \mathrm{mmol}, 77 \%)$.

$\mathbf{R}_{\mathbf{f}}($ pentane $/$ EtOAc 80:20) $=0.39$. 
${ }^{1} \mathbf{H}$ NMR (400 MHz, $\left.\mathrm{CDCl}_{3}\right): \delta(\mathrm{ppm}) 7.29-7.20(\mathrm{~m}, 3 \mathrm{H}), 7.18-7.14(\mathrm{~m}, 1 \mathrm{H}), 4.65(\mathrm{~s}, 1 \mathrm{H}), 4.35-$ $4.20(\mathrm{~m}, 2 \mathrm{H}), 3.97(\mathrm{~s}, 3 \mathrm{H}), 2.90(\mathrm{td}, J=13.0,4.5 \mathrm{~Hz}, 1 \mathrm{H}), 2.72(\mathrm{td}, J=12.9,4.8 \mathrm{~Hz}, 1 \mathrm{H}), 2.17(\mathrm{~s}, 3 \mathrm{H})$, 2.07 (td, $J=13.1,4.5 \mathrm{~Hz}, 1 \mathrm{H}), 1.86(\mathrm{td}, J=13.3,4.8 \mathrm{~Hz}, 1 \mathrm{H}), 1.64(\mathrm{~s}, 3 \mathrm{H}), 1.28$ (t, $J=7.2 \mathrm{~Hz}, 3 \mathrm{H})$.

${ }^{13} \mathbf{C}$ NMR (101 MHz, $\left.\mathrm{CDCl}_{3}\right): \delta$ (ppm) 172.1, 167.3, 156.0, 138.6, 137.3, 130.1, 128.8, 128.7, 126.7, $77.6,62.2,61.9,61.3,33.9,28.3,19.5,16.7,14.3$.

HRMS (ESI): calculated for $\mathrm{C}_{18} \mathrm{H}_{23} \mathrm{NO}_{5} \mathrm{Na}[\mathrm{M}+\mathrm{Na}]^{+}:$356.1468; found: 356.1468 .

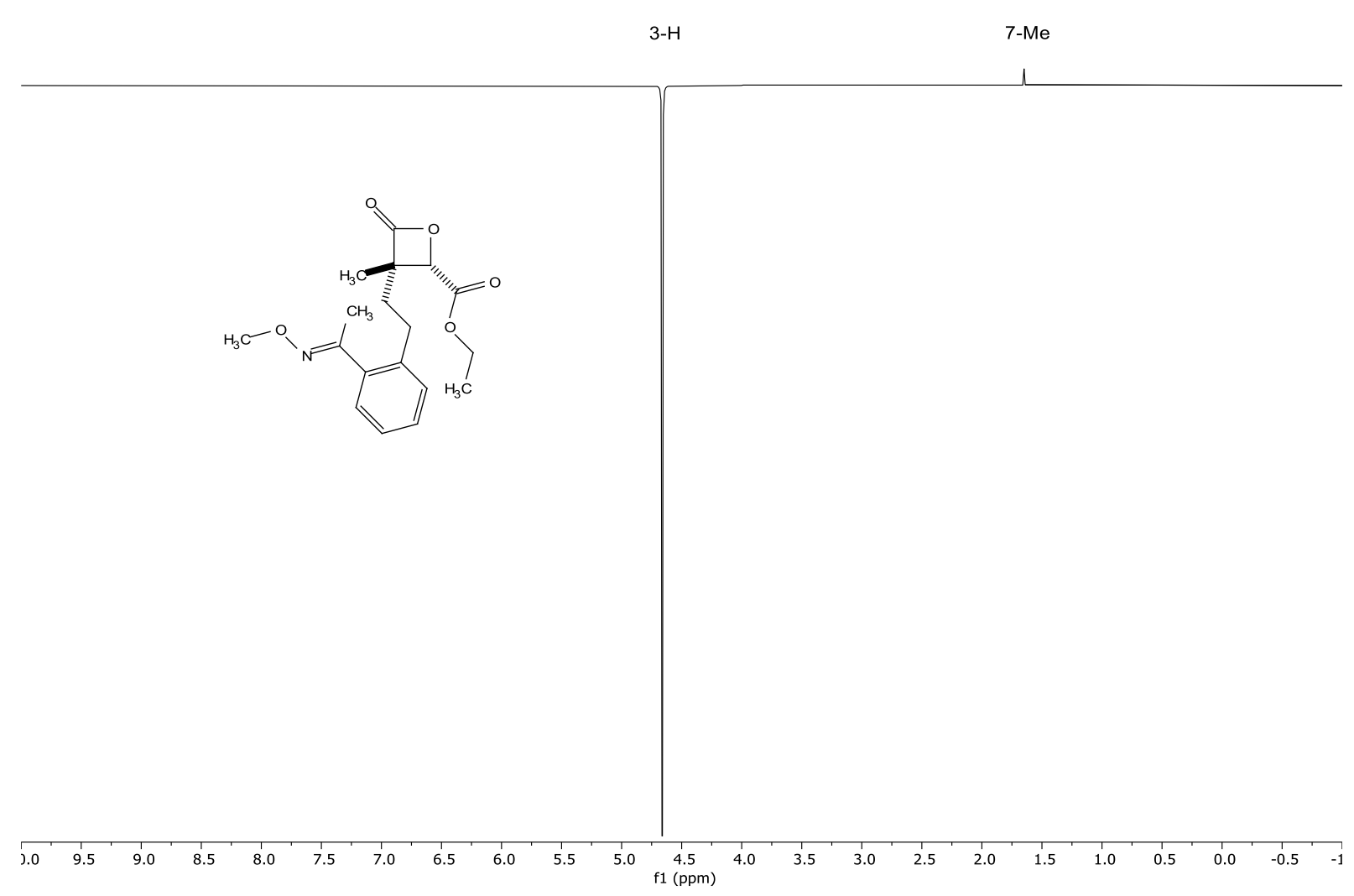

Figure S2. NOE experiment of compound 6 irradiating $\delta_{\mathrm{H}}=4.65 \mathrm{ppm}$. 


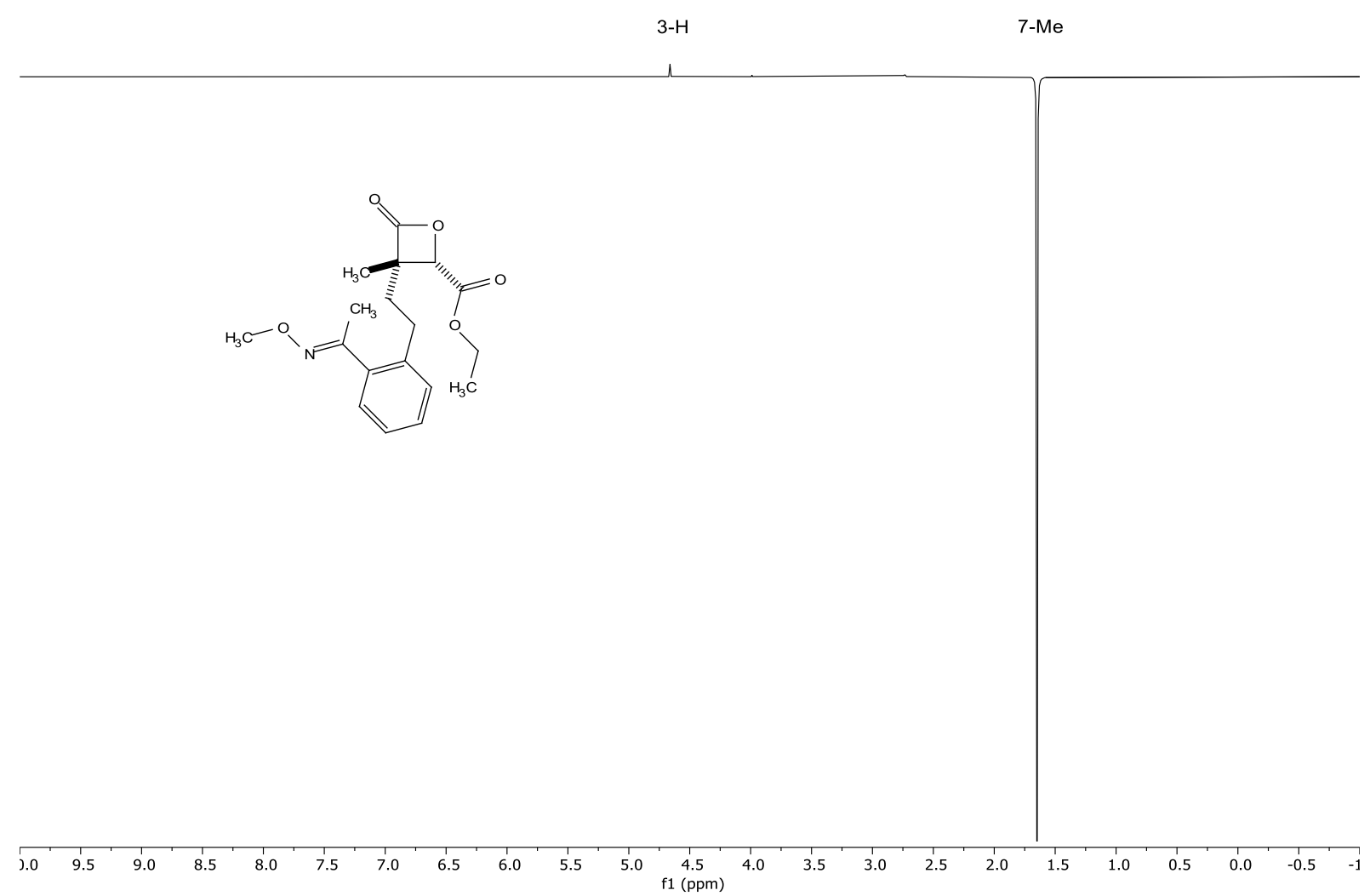

Figure S3. NOE experiment of compound 6 irradiating $\delta_{\mathrm{H}}=1.64 \mathrm{ppm}$.

Although we observed a significant NOE supporting the indicated stereochemistry, we were interested in synthesizing the respective $\mathrm{C} 3$-epimer.

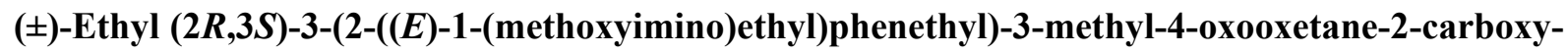
late (S1)<smiles>CCOC(=O)[C@H](O)[C@@](C)(CCc1ccccc1/C(C)=N/OC)C(=O)O</smiles>

5

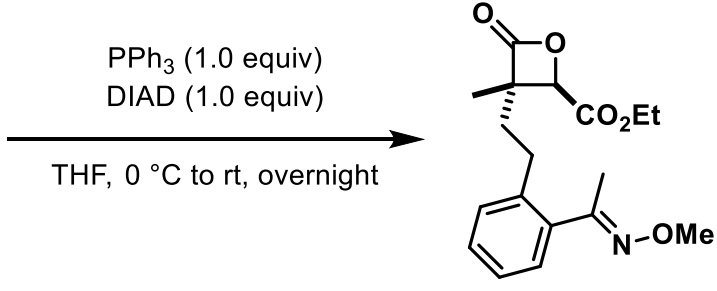

S1

According to a procedure from Barbaud ${ }^{12}$ compound 5 (36.6 mg, $0.10 \mathrm{mmol}, 1.0$ equiv) and triphenylphosphine $(27.3 \mathrm{mg}, 0.10 \mathrm{mmol}, 1.0$ equiv) were dissolved in THF $(0.35 \mathrm{~mL}, 0.3 \mathrm{M})$ and cooled to $0{ }^{\circ} \mathrm{C}$. DIAD $(20.5 \mu \mathrm{L}, 0.10 \mathrm{mmol}, 1.0$ equiv) was added and the mixture was stirred for $1 \mathrm{~h}$ at $0{ }^{\circ} \mathrm{C}$. The reaction was allowed to warm to room temperature and was stirred overnight. The solvent was evaporated and the residue was redissolved in cold diethyl ether and filtered. Purification by column chromatography (eluent: pentane/EtOAc) yielded the desired product as a pale yellow oil $(10.9 \mathrm{mg}$, $0.03 \mathrm{mmol}, 31 \%)$.

$\mathbf{R}_{\mathbf{f}}($ pentane/EtOAc 85:15) $=0.31$. 
${ }^{1} \mathbf{H}$ NMR $\left(599 \mathrm{MHz}, \mathrm{CDCl}_{3}\right): \delta(\mathrm{ppm}) 7.29(\mathrm{ddd}, J=7.5,6.7,2.4 \mathrm{~Hz}, 1 \mathrm{H}), 7.27-7.24(\mathrm{~m}, 2 \mathrm{H}), 7.24-$ $7.21(\mathrm{~m}, 1 \mathrm{H}), 4.76(\mathrm{~s}, 1 \mathrm{H}), 4.33(\mathrm{q}, J=7.1 \mathrm{~Hz}, 2 \mathrm{H}), 3.97(\mathrm{~s}, 3 \mathrm{H}), 2.96-2.87(\mathrm{~m}, 1 \mathrm{H}), 2.80-2.71(\mathrm{~m}$, 1H), 2.20 (s, 3H), $2.18-2.08(\mathrm{~m}, 2 \mathrm{H}), 1.37-1.32(\mathrm{~m}, 6 \mathrm{H})$.

${ }^{13} \mathbf{C}$ NMR (151 MHz, $\left.\mathrm{CDCl}_{3}\right): \delta$ (ppm) 172.1, 167.6, 156.2, 138.2, 137.2, 130.2, 128.9, 128.8, 126.8, $75.5,62.1,61.9,61.9,37.5,28.8,16.7,15.6,14.4$.

HRMS (ESI): calculated for $\mathrm{C}_{18} \mathrm{H}_{23} \mathrm{NO}_{5} \mathrm{Na}[\mathrm{M}+\mathrm{Na}]^{+}:$356.1468; found: 356.1466 .

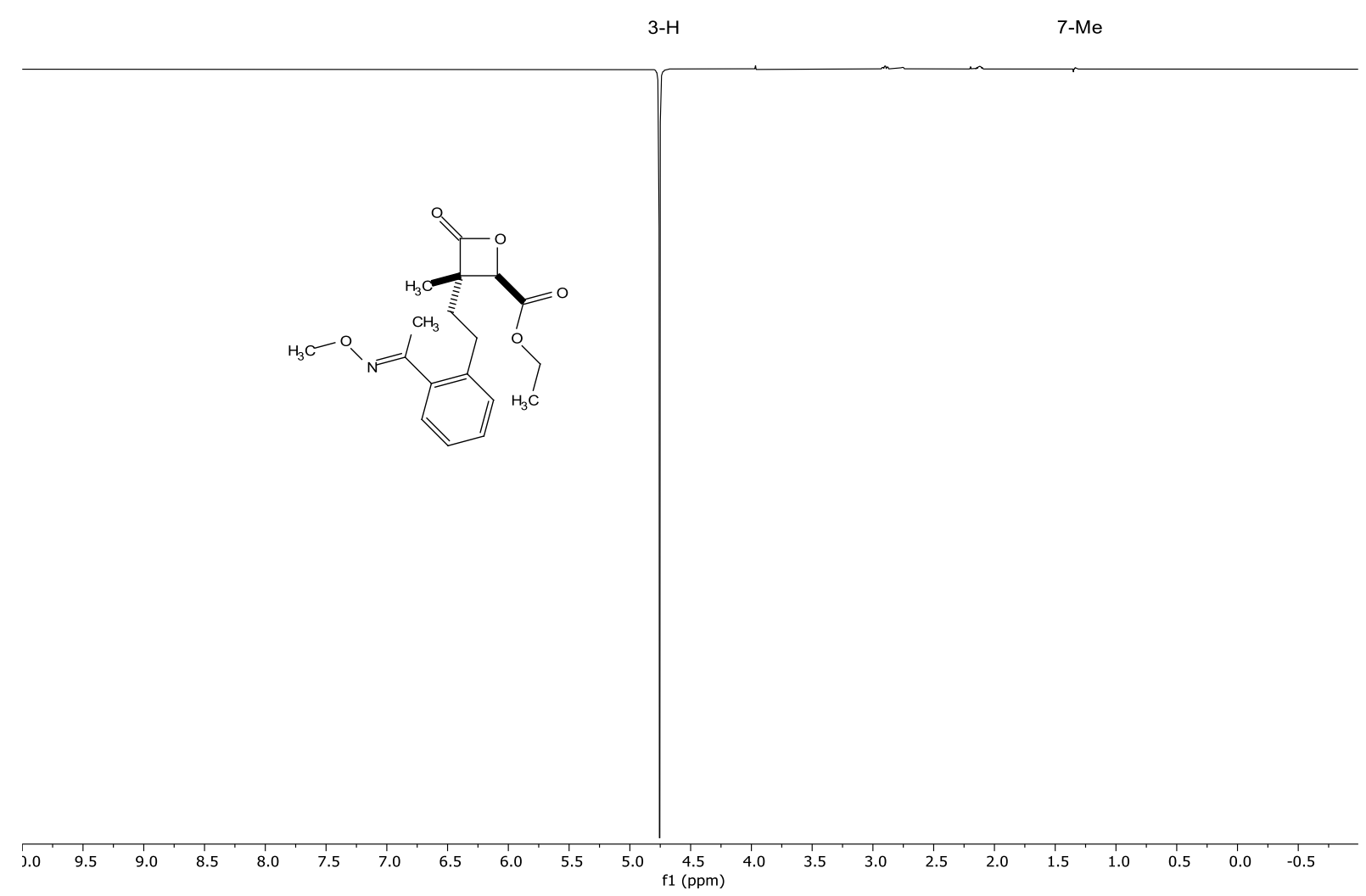

Figure S4. NOE experiment of compound S1 irradiating $\delta_{\mathrm{H}}=4.76 \mathrm{ppm}$. 


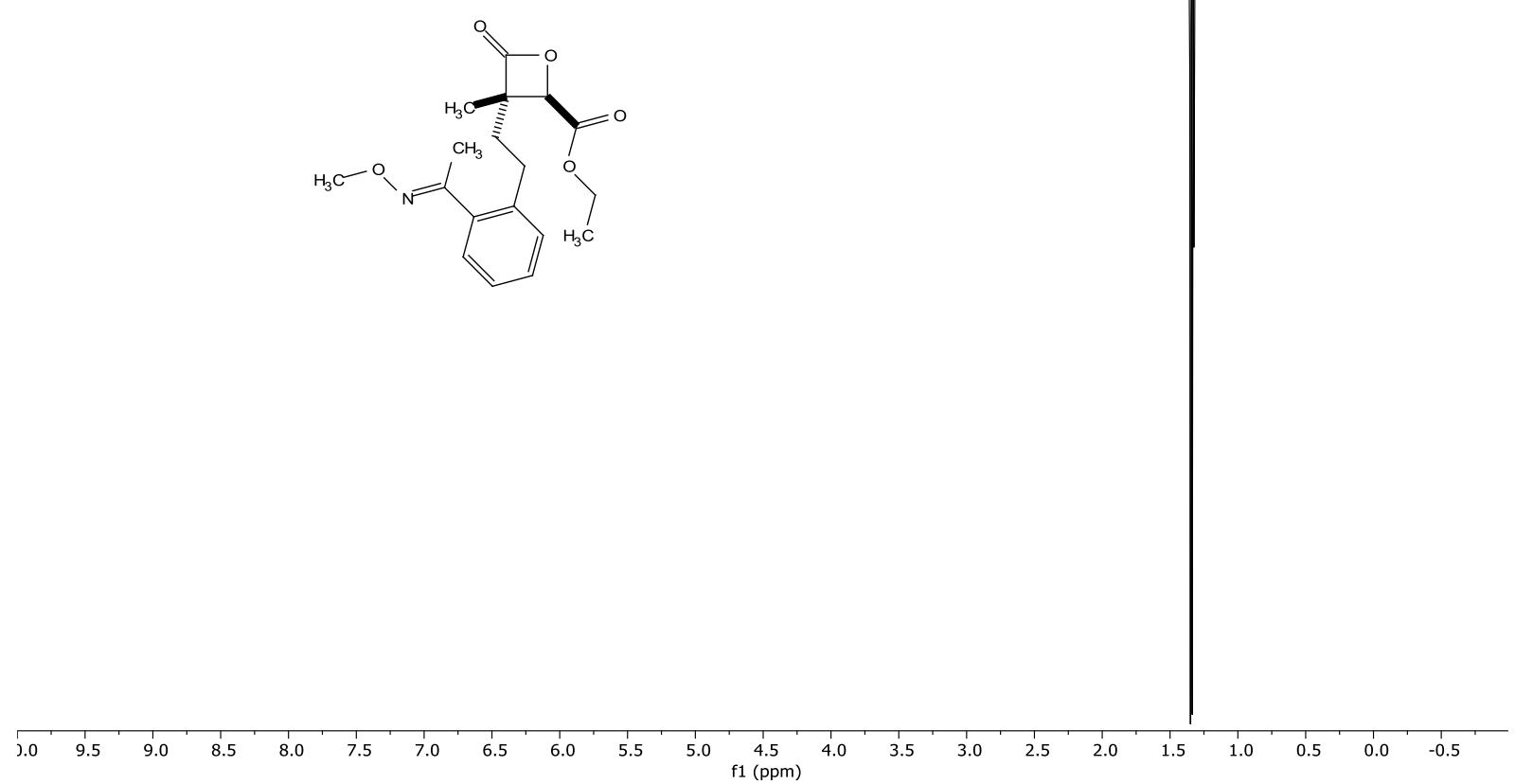

Figure S5. NOE experiment of compound S1 irradiating $\delta_{\mathrm{H}}=1.37-1.32 \mathrm{ppm}$. 


\section{Mechanistic Experiments}

\subsection{Parallel KIE Experiment}

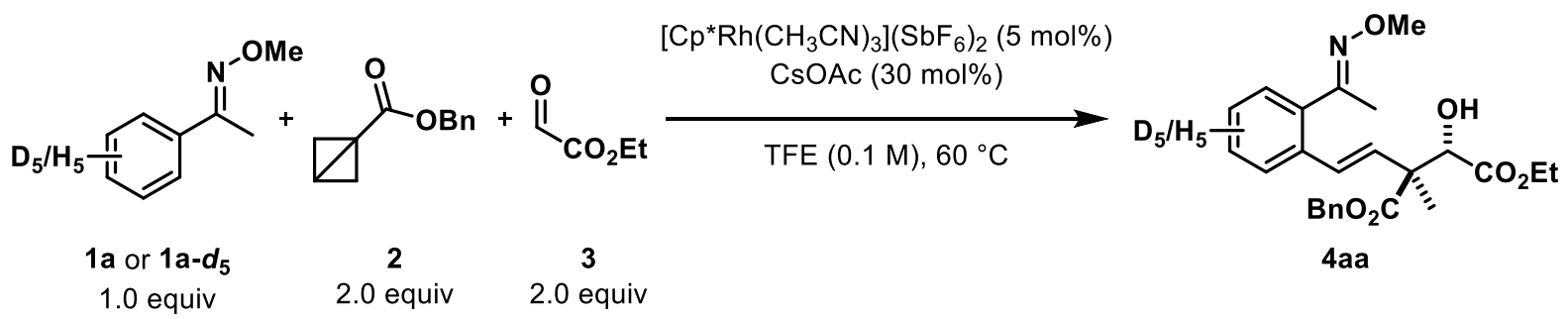

Two parallel experiments were conducted. An oven-dried $10 \mathrm{~mL}$ Schlenk tube equipped with a stir bar was charged with $\mathrm{CsOAc}\left(11.5 \mathrm{mg}, 0.06 \mathrm{mmol}, 0.3\right.$ equiv) in a glovebox. $\left[\mathrm{Cp} * \mathrm{Rh}\left(\mathrm{CH}_{3} \mathrm{CN}\right)_{3}\right]\left(\mathrm{SbF}_{6}\right)_{2}$ (8.3 $\mathrm{mg}, 0.01 \mathrm{mmol}, 5 \mathrm{~mol} \%)$, TFE $(2 \mathrm{~mL}, 0.1 \mathrm{M})$, 1a $\left(29.8 \mathrm{mg}, 0.20 \mathrm{mmol}, 1.0\right.$ equiv) or $\mathbf{1 a -} \boldsymbol{d}_{5}$ (30.8 mg, $0.2 \mathrm{mmol}, 1.0$ equiv), benzyl bicyclo[1.1.0]butane-1-carboxylate 2a (75.3 $\mathrm{mg}, 0.40 \mathrm{mmol}$, 2.0 equiv), ethyl glyoxylate $3(81.5 \mu \mathrm{L}$ of a $50 \mathrm{wt} \%$ solution in toluene, $0.4 \mathrm{mmol}, 2.0$ equiv) and 1fluoronaphthalene $(26.0 \mu \mathrm{L}, 0.20 \mathrm{mmol})$ were added sequentially under a positive stream of argon. The tube was sealed and the reaction was stirred for $80 \mathrm{~min}$ at $60^{\circ} \mathrm{C}$. An aliquot $(5 \mu \mathrm{L})$ was taken after 20, 40, 60 and 80 minutes respectively, filtered through a plug of Celite and analyzed by LC-UV.

Table S2. Parallel KIE experiment.

\begin{tabular}{|c|c|c|}
\hline time (min) & yield of 4aa using $\mathbf{1 a}(\boldsymbol{\%})$ & yield of $\mathbf{4 a a}$ using $\mathbf{1 a}-\boldsymbol{d}_{\mathbf{5}}(\mathbf{\%})$ \\
\hline 20 & 5 & 4 \\
\hline 40 & 10 & 9 \\
\hline 60 & 16 & 13 \\
\hline 80 & 21 & 18 \\
\hline
\end{tabular}

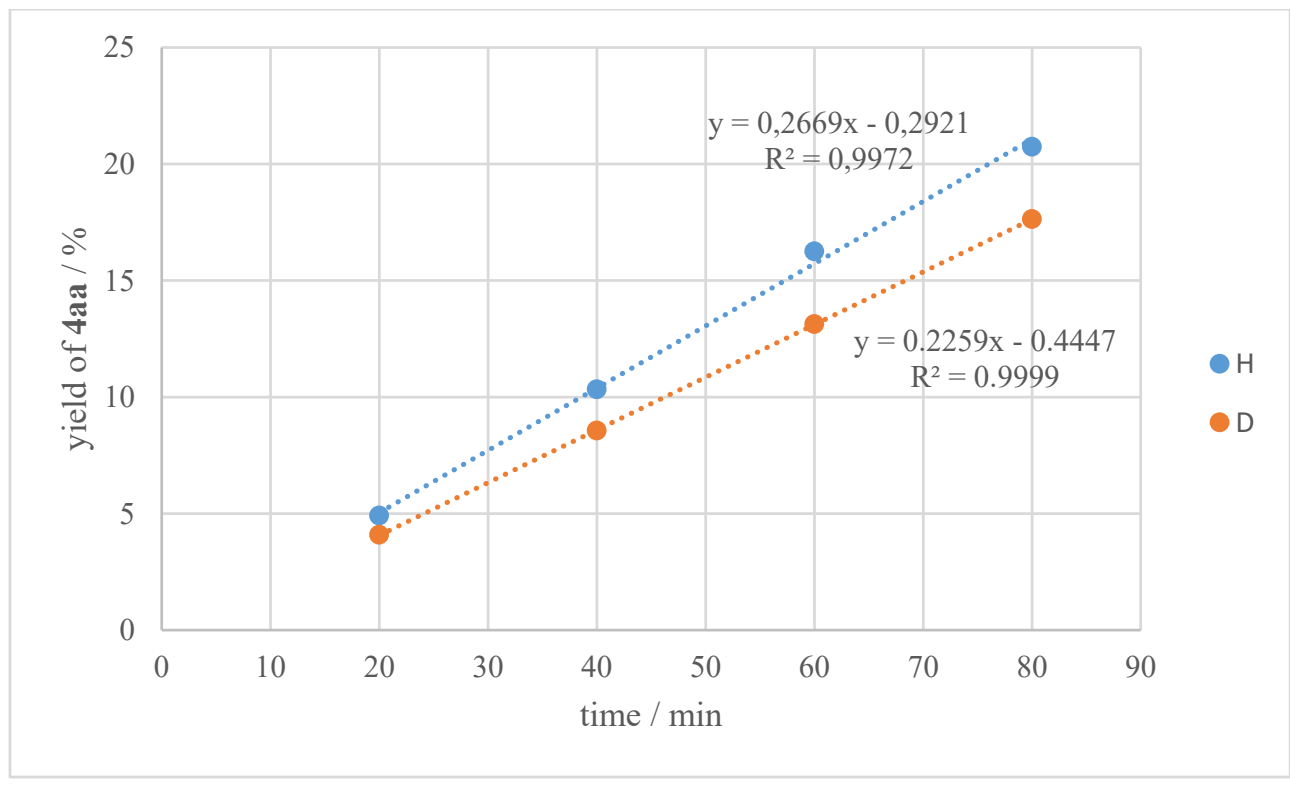

Figure S6. Parallel KIE experiment.

The $k_{\mathrm{H}} / k_{\mathrm{D}}$ value was determined to be 1.2 . 


\subsection{Two-Component Experiments}

\section{Benzyl (E)-4-(2-((E)-1-(methoxyimino)ethyl)phenyl)-2-methylbut-3-enoate (7)}

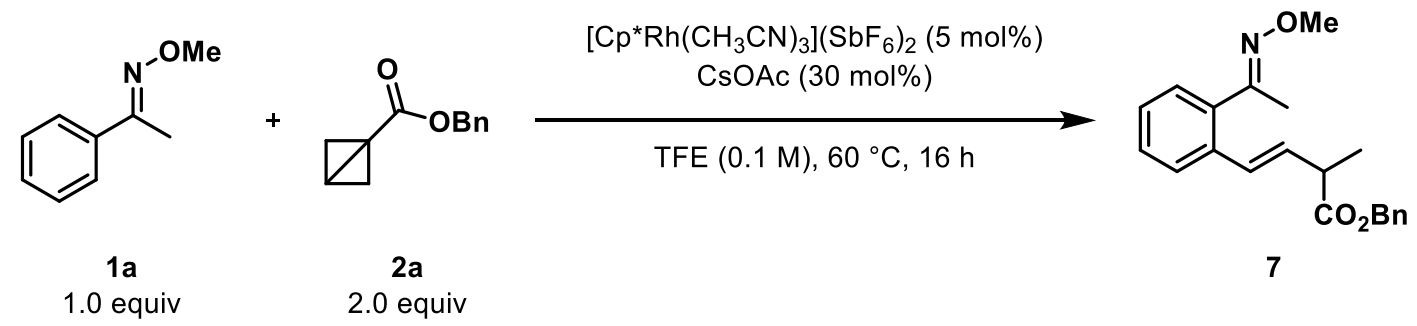

An oven-dried $10 \mathrm{~mL}$ Schlenk tube equipped with a stir bar was charged with CsOAc (11.5 mg, $0.06 \mathrm{mmol}, 0.3$ equiv) in a glovebox. [Cp* $\left.\mathrm{Rh}\left(\mathrm{CH}_{3} \mathrm{CN}\right)_{3}\right]\left(\mathrm{SbF}_{6}\right)_{2}(8.3 \mathrm{mg}, 0.01 \mathrm{mmol}, 5 \mathrm{~mol} \%)$, TFE $(2 \mathrm{~mL}, 0.1 \mathrm{M}),(E)-1$-phenylethan-1-one $O$-methyl oxime 1a $(29.8 \mathrm{mg}, 0.20 \mathrm{mmol}, 1.0$ equiv) and benzyl bicyclo[1.1.0]butane-1-carboxylate $2 \mathbf{a}(75.3 \mathrm{mg}, 0.40 \mathrm{mmol}, 2.0$ equiv) were added sequentially under a positive stream of argon. The tube was sealed and the reaction was stirred for $16 \mathrm{~h}$ at $60{ }^{\circ} \mathrm{C}$. The reaction was cooled to room temperature and purification by column chromatography (eluent: pentane/EtOAc) yielded the desired product as an orange sticky oil (13.1 mg, $0.04 \mathrm{mmol}, 19 \%)$.

$\mathbf{R}_{\mathbf{f}}($ pentane/EtOAc 90:10) $=0.43$.

${ }^{1} \mathbf{H}$ NMR $\left(400 \mathrm{MHz}, \mathrm{CDCl}_{3}\right): \delta(\mathrm{ppm}) 7.41-7.36(\mathrm{~m}, 1 \mathrm{H}), 7.28-7.19(\mathrm{~m}, 6 \mathrm{H}), 7.18-7.15(\mathrm{~m}, 2 \mathrm{H})$, $6.56(\mathrm{~d}, J=15.8 \mathrm{~Hz}, 1 \mathrm{H}), 6.12(\mathrm{dd}, J=15.8,8.0 \mathrm{~Hz}, 1 \mathrm{H}), 5.06$ (s, 2H), 3.88 (s, 3H), $3.34-3.24$ (m, $1 \mathrm{H}), 2.03(\mathrm{~s}, 3 \mathrm{H}), 1.30(\mathrm{~d}, J=7.1 \mathrm{~Hz}, 3 \mathrm{H})$.

${ }^{13}$ C NMR (101 MHz, $\left.\mathrm{CDCl}_{3}\right): \delta(\mathrm{ppm}) 174.3,156.8,136.4,136.1,135.4,130.5,129.7,128.8,128.7$, $128.3,128.2,127.6,126.7,66.6,62.0,43.5,17.4,17.0$, one carbon signal missing due to overlap.

HRMS (ESI): calculated for $\mathrm{C}_{21} \mathrm{H}_{23} \mathrm{NO}_{3} \mathrm{Na}[\mathrm{M}+\mathrm{Na}]^{+}: 360.1570$; found: 360.1573 .

Ethyl (E)-2-hydroxy-2-(2-(1-(methoxyimino)ethyl)phenyl)acetate (8)

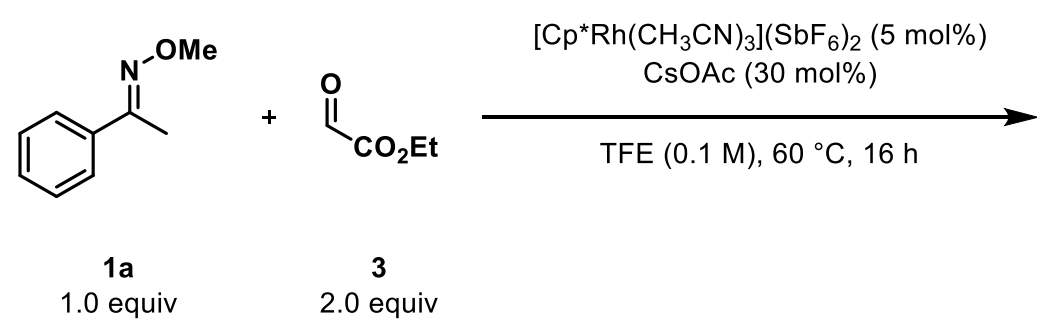

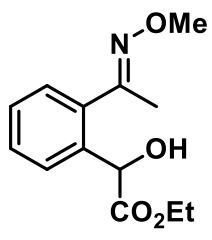

8

An oven-dried $10 \mathrm{~mL}$ Schlenk tube equipped with a stir bar was charged with CsOAc $(11.5 \mathrm{mg}$, $0.06 \mathrm{mmol}, 0.3$ equiv) in a glovebox. [Cp* $\left.\mathrm{Rh}\left(\mathrm{CH}_{3} \mathrm{CN}\right)_{3}\right]\left(\mathrm{SbF}_{6}\right)_{2}(8.3 \mathrm{mg}, 0.01 \mathrm{mmol}, 5 \mathrm{~mol} \%)$, TFE $(2 \mathrm{~mL}, 0.1 \mathrm{M}),(E)$-1-phenylethan-1-one $O$-methyl oxime $1 \mathrm{a}(29.8 \mathrm{mg}, 0.20 \mathrm{mmol}, 1.0$ equiv) and ethyl glyoxylate $3(81.5 \mu \mathrm{L}$ of a $50 \mathrm{wt} \%$ solution in toluene, $0.4 \mathrm{mmol}, 2.0$ equiv) were added sequentially under a positive stream of argon. The tube was sealed and the reaction was stirred for $16 \mathrm{~h}$ at $60{ }^{\circ} \mathrm{C}$. The reaction was cooled to room temperature and purification by column chromatography (eluent: pentane/EtOAc) yielded the desired product as an orange sticky oil (34.0 mg, $0.14 \mathrm{mmol}, 68 \%$ ).

$\mathbf{R}_{\mathbf{f}}($ pentane/EtOAc 80:20) $=0.27$.

${ }^{1}$ H NMR $\left(400 \mathrm{MHz}, \mathrm{CDCl}_{3}\right): \delta(\mathrm{ppm}) 7.40-7.36(\mathrm{~m}, 4 \mathrm{H}), 5.19(\mathrm{~s}, 1 \mathrm{H}), 4.23-4.14(\mathrm{~m}, 2 \mathrm{H}), 4.01$ (s, $3 \mathrm{H}), 2.22(\mathrm{~s}, 3 \mathrm{H}), 1.23(\mathrm{t}, J=7.1 \mathrm{~Hz}, 3 \mathrm{H})$, one proton signal missing due to broadening. 
${ }^{13} \mathrm{C}$ NMR (101 MHz, $\left.\mathrm{CDCl}_{3}\right): \delta(\mathrm{ppm}) 173.2,157.0,137.9,136.2,131.0,129.4,129.0,128.7,74.2$, $62.1,61.6,15.8,14.3$.

HRMS (ESI): calculated for $\mathrm{C}_{13} \mathrm{H}_{17} \mathrm{NO}_{4} \mathrm{Na}[\mathrm{M}+\mathrm{Na}]^{+}: 274.1050$; found: 274.1051 .

\subsection{Stepwise Mechanism?}<smiles>CO/N=C(\C)c1ccccc1/C=C/C(C)C(=O)OCc1ccccc1</smiles>

7

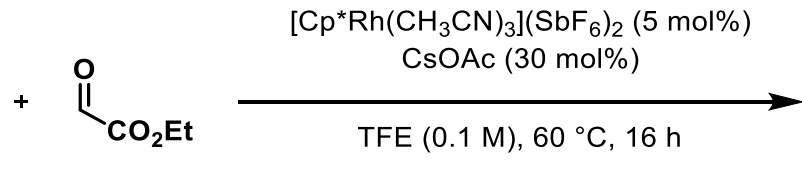

3

2.0 equiv<smiles>CCOC(=O)[C@H](O)[C@@](C)(/C=C/c1ccccc1/C(C)=N/OC)C(=O)OCC</smiles>

4aa

$7: 1 \mathrm{dr}$

An oven-dried $10 \mathrm{~mL}$ Schlenk tube equipped with a stir bar was charged with CsOAc $(5.8 \mathrm{mg}$, $0.03 \mathrm{mmol}, 0.3$ equiv) in a glovebox. $\left[\mathrm{Cp} * \mathrm{Rh}\left(\mathrm{CH}_{3} \mathrm{CN}\right)_{3}\right]\left(\mathrm{SbF}_{6}\right)_{2}(4.2 \mathrm{mg}, 0.005 \mathrm{mmol}, 5 \mathrm{~mol} \%)$, TFE $(1 \mathrm{~mL}, 0.1 \mathrm{M})$, compound $7(33.7 \mathrm{mg}, 0.10 \mathrm{mmol}, 1.0$ equiv) and ethyl glyoxylate $3(40.5 \mu \mathrm{L}$ of a $50 \mathrm{wt} \%$ solution in toluene, $0.2 \mathrm{mmol}, 2.0$ equiv) were added sequentially under a positive stream of argon. The tube was sealed and the reaction was stirred for $16 \mathrm{~h}$ at $60{ }^{\circ} \mathrm{C}$. The reaction was cooled to room temperature and 1-fluoronaphthalene $(13.0 \mu \mathrm{L}, 0.10 \mathrm{mmol})$ was added as an internal standard. An aliquot $(5 \mu \mathrm{L})$ was taken, filtered through a plug of Celite and analyzed by LC-UV. The desired product was detected in $12 \%$ yield with $7: 1 \mathrm{dr}$.

\subsection{Isomerization Experiment}

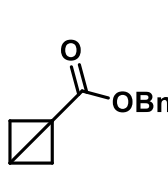

$2 a$

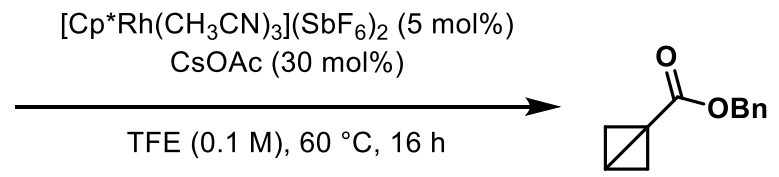

2a

An oven-dried $10 \mathrm{~mL}$ Schlenk tube equipped with a stir bar was charged with CsOAc $(5.8 \mathrm{mg}$, $0.03 \mathrm{mmol}, 0.3$ equiv) in a glovebox. $\left[\mathrm{Cp} * \mathrm{Rh}\left(\mathrm{CH}_{3} \mathrm{CN}\right)_{3}\right]\left(\mathrm{SbF}_{6}\right)_{2}(4.2 \mathrm{mg}, 0.005 \mathrm{mmol}, 5 \mathrm{~mol} \%)$, TFE $(1 \mathrm{~mL}, 0.1 \mathrm{M})$, benzyl bicyclo[1.1.0]butane-1-carboxylate 2a $(37.6 \mathrm{mg}, 0.20 \mathrm{mmol}, 2.0$ equiv) were added sequentially under a positive stream of argon. The tube was sealed and the reaction was stirred for $16 \mathrm{~h}$ at $60{ }^{\circ} \mathrm{C}$. The reaction was cooled to room temperature and trimethoxybenzene $(16.8 \mathrm{mg}$, $0.10 \mathrm{mmol}$ ) was added as an internal standard. The reaction mixture was filtered through a plug of Celite and submitted to ${ }^{1} \mathrm{H}$ NMR analysis. The starting material could be recovered in $83 \%$ yield with no other isomers detected. However, we assume that the loss of material can be attributed to polymerization processes that are known to occur by radical as well as anionic initiation. ${ }^{13}$ 


\section{Proof of the Stereochemistry by X-Ray Crystallography}

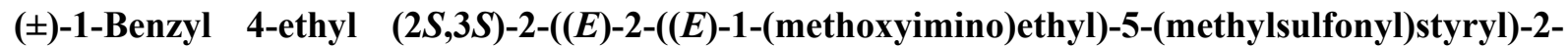
methyl-3-((4-(methylsulfonyl)benzoyl)oxy)succinate (S2)

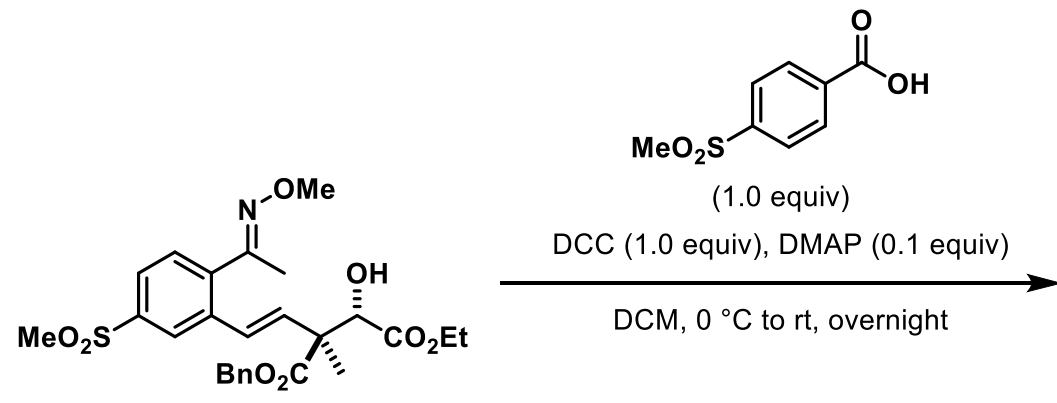

4sa (1.1 equiv)<smiles>CCOC(=O)c1ccc(S(C)(=O)=O)cc1C(=O)O[C@@H](C(C)=O)C(C)(C)C=Cc1cc(OC)ccc1/C(C)=N/OC</smiles>

S2

Based on a modified procedure from Bannwarth ${ }^{14} 4$-(methylsulfonyl)benzoic acid ( $26.2 \mathrm{mg}, 0.13 \mathrm{mmol}$, 1.0 equiv), DMAP ( $1.6 \mathrm{mg}, 0.013 \mathrm{mmol}, 0.1$ equiv) and compound 4sa (74.6 mg, $0.144 \mathrm{mmol}$, 1.1 equiv) were dissolved in DCM $(0.66 \mathrm{~mL}, 0.2)$ and cooled to $0{ }^{\circ} \mathrm{C}$. DCC $(27.0 \mathrm{mg}, 0.13 \mathrm{mmol}$, 1.0 equiv) was added and the mixture was allowed to warm to room temperature. The reaction was stirred overnight and then filtered through a plug of Celite. Purification by column chromatography (eluent: pentane/EtOAc) yielded the desired product as a turbid sticky oil (78.5 mg, $0.11 \mathrm{mmol}, 86 \%$ ).

$\mathbf{R}_{\mathbf{f}}($ pentane/EtOAc 50:50) $=0.29$.

${ }^{1} \mathbf{H}$ NMR $\left(400 \mathrm{MHz}, \mathrm{CDCl}_{3}\right): \delta(\mathrm{ppm}) 8.16-8.10(\mathrm{~m}, 2 \mathrm{H}), 8.00-7.97(\mathrm{~m}, 2 \mathrm{H}), 7.93(\mathrm{~d}, J=1.9 \mathrm{~Hz}$, $1 \mathrm{H}), 7.81(\mathrm{dd}, J=8.1,1.9 \mathrm{~Hz}, 1 \mathrm{H}), 7.47(\mathrm{~d}, J=8.1 \mathrm{~Hz}, 1 \mathrm{H}), 7.37-7.33(\mathrm{~m}, 2 \mathrm{H}), 7.33-7.24(\mathrm{~m}, 3 \mathrm{H})$, $6.81(\mathrm{~d}, J=16.2 \mathrm{~Hz}, 1 \mathrm{H}), 6.48(\mathrm{~d}, J=16.2 \mathrm{~Hz}, 1 \mathrm{H}), 5.84(\mathrm{~s}, 1 \mathrm{H}), 5.27-5.16(\mathrm{~m}, 2 \mathrm{H}), 4.24-4.08(\mathrm{~m}$, 2H), 3.97 (s, 3H), 3.07 (s, 3H), $3.01(\mathrm{~s}, 3 \mathrm{H}), 2.09$ (s, 3H), $1.64(\mathrm{~s}, 3 \mathrm{H}), 1.19(\mathrm{t}, J=7.1 \mathrm{~Hz}, 3 \mathrm{H})$.

${ }^{13} \mathrm{C}$ NMR (101 MHz, $\left.\mathrm{CDCl}_{3}\right): \delta$ (ppm) 171.3, 166.9, 164.0, 154.6, 144.8, 141.3, 140.8, 136.6, 135.2, $133.7,132.1,130.8,130.0,129.2,128.7,128.6,128.6,127.8,126.5,125.8,76.7,67.8,62.3,62.2,51.6$, $44.5,44.4,17.4,16.3,14.1$.

HRMS (ESI): calculated for $\mathrm{C}_{34} \mathrm{H}_{37} \mathrm{NO}_{11} \mathrm{~S}_{2} \mathrm{Na}[\mathrm{M}+\mathrm{Na}]^{+}:$722.1700; found: 722.1701 .

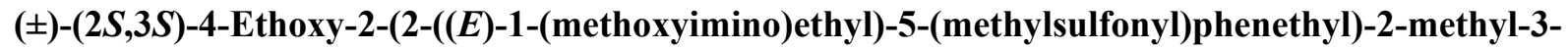
((4-(methylsulfonyl)benzoyl)oxy)-4-oxobutanoic acid (S3)<smiles>CCOC(OC(=O)c1ccc(S(C)(=O)=O)cc1)C(C)(C)C(C)(C)OCc1cc(OC)ccc1/C(C)=N/OC</smiles>

S2

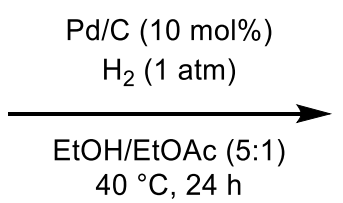

$40^{\circ} \mathrm{C}, 24 \mathrm{~h}$<smiles>CCOC(=O)c1ccc(S(C)(=O)=O)cc1</smiles>

S3

Based on a modified procedure from Proudfoot ${ }^{10}$ an oven-dried $10 \mathrm{~mL}$ Schlenk tube equipped with a stir bar was charged with Pd/C (10 wt\%, $9.3 \mathrm{mg}, 0.009 \mathrm{mmol}, 10 \mathrm{~mol} \%)$. Compound S2 $(61.0 \mathrm{mg}$, $0.09 \mathrm{mmol}, 1.0$ equiv) was added as a solution in EtOH/EtOAc $(5: 1,0.44 \mathrm{~mL}, 0.2 \mathrm{M})$ and the reaction vessel was purged with $\mathrm{H}_{2}$. The reaction was then stirred at $40{ }^{\circ} \mathrm{C}$ for $24 \mathrm{~h}$ under a $\mathrm{H}_{2}$ atmosphere. After cooling to room temperature the mixture was filtered through a plug of Celite and purification by 
preparative TLC (eluent: DCM/EtOH) yielded the desired product as a white solid (47.0 $\mathrm{mg}, 0.08 \mathrm{mmol}$, $88 \%$ ).

$\mathbf{R}_{\mathbf{f}}(\mathrm{DCM} / \mathrm{EtOH} 90: 10)=0.54$

${ }^{1} \mathbf{H}$ NMR $\left(400 \mathrm{MHz}, \mathrm{CDCl}_{3}\right): \delta(\mathrm{ppm}) 8.22(\mathrm{~d}, J=8.0 \mathrm{~Hz}, 2 \mathrm{H}), 8.04(\mathrm{~d}, J=8.2 \mathrm{~Hz}, 2 \mathrm{H}), 7.81-7.71(\mathrm{~m}$, 2H), $7.37(\mathrm{~d}, J=8.0 \mathrm{~Hz}, 1 \mathrm{H}), 5.73(\mathrm{~s}, 1 \mathrm{H}), 4.30-4.15(\mathrm{~m}, 2 \mathrm{H}), 3.94(\mathrm{~s}, 3 \mathrm{H}), 3.08(\mathrm{~s}, 3 \mathrm{H}), 3.04-2.93$ (m, 4H), $2.82-2.71(\mathrm{~m}, 1 \mathrm{H}), 2.18-2.04(\mathrm{~m}, 4 \mathrm{H}), 2.02-1.92(\mathrm{~m}, 1 \mathrm{H}), 1.42(\mathrm{~s}, 3 \mathrm{H}), 1.23(\mathrm{t}, J=7.3 \mathrm{~Hz}$, $3 \mathrm{H})$, one proton signal missing due to broadening.

${ }^{13} \mathrm{C}$ NMR (101 MHz, $\left.\mathrm{CDCl}_{3}\right): \delta$ (ppm) 177.9, 167.6, 164.2, 154.8, 145.0, 142.7, 141.2, 140.5, 133.9, $130.9,129.9,129.0,127.9,125.5,77.4,62.4,62.2,49.1,44.6,44.4,38.1,28.7,17.4,16.6,14.1$.

HRMS (ESI): calculated for $\mathrm{C}_{27} \mathrm{H}_{32} \mathrm{NO}_{11} \mathrm{~S}_{2} \mathrm{Na}_{2}[\mathrm{M}-\mathrm{H}+2 \mathrm{Na}]^{+}$: 656.1207; found: 656.1208.

\section{Crystallographic Data}

In order to provide evidence for the diastereoselectivity of the developed reaction, we determined the crystal structure of compound $\mathbf{S 3}$. As the result, the anti configuration was unambiguously assigned.

X-ray crystal structure analysis of S3: A colorless needle-like specimen of $\mathrm{C}_{27} \mathrm{H}_{33} \mathrm{NO}_{11} \mathrm{~S}_{2}$, approximate dimensions $0.046 \mathrm{~mm} \times 0.067 \mathrm{~mm} \times 0.246 \mathrm{~mm}$, was used for the X-ray crystallographic analysis. The X-ray intensity data were measured on a Bruker D8 Venture Bruker D8 Venture Photon III Diffractometer system equipped with a micro focus tube $\mathrm{CuK} \alpha(\mathrm{CuK} \alpha, \lambda=1.54178 \AA$ ) and a $\mathrm{MX}$ mirror monochromator. A total of 2360 frames were collected. The total exposure time was 20.51 hours. The frames were integrated with the Bruker SAINT software package using a wide-frame algorithm. The integration of the data using a monoclinic unit cell yielded a total of 56809 reflections to a maximum $\theta$ angle of $68.28^{\circ}(0.83 \AA$ resolution), of which 5282 were independent (average redundancy 10.755, completeness $\left.=99.4 \%, \mathrm{R}_{\text {int }}=5.35 \%, \mathrm{R}_{\text {sig }}=2.44 \%\right)$ and $4648(88.00 \%)$ were greater than $2 \sigma\left(\mathrm{F}^{2}\right)$. The final cell constants of $\underline{a}=24.4340(5) \AA, \underline{b}=7.08410(10) \AA, \underline{c}=34.0203(7) \AA, \beta=100.9880(10)^{\circ}$, volume $=5780.72(19) \AA^{3}$, are based upon the refinement of the XYZ-centroids of 9888 reflections above $20 \sigma(\mathrm{I})$ with $7.371^{\circ}<2 \theta<136.4^{\circ}$. Data were corrected for absorption effects using the Multi-Scan method (SADABS). The ratio of minimum to maximum apparent transmission was 0.849 . The calculated minimum and maximum transmission coefficients (based on crystal size) are 0.6130 and 0.9060. The structure was solved and refined using the Bruker SHELXTL Software Package, using the space group $C 2 / c$, with $\mathrm{Z}=8$ for the formula unit, $\mathrm{C}_{27} \mathrm{H}_{33} \mathrm{NO}_{11} \mathrm{~S}_{2}$. The final anisotropic full-matrix leastsquares refinement on $\mathrm{F}^{2}$ with 380 variables converged at $\mathrm{R} 1=3.14 \%$, for the observed data and $\mathrm{wR} 2$ $=7.59 \%$ for all data. The goodness-of-fit was 1.038 . The largest peak in the final difference electron density synthesis was $0.454 \mathrm{e}^{-} / \AA^{3}$ and the largest hole was $-0.342 \mathrm{e}^{-} / \AA^{3}$ with an RMS deviation of 0.045 $\mathrm{e}^{-} / \AA^{3}$. On the basis of the final model, the calculated density was $1.406 \mathrm{~g} / \mathrm{cm}^{3}$ and $\mathrm{F}(000), 2576 \mathrm{e}^{-}$. The hydrogen at O5 atom was refined freely. CCDC Nr.: 2070494. 


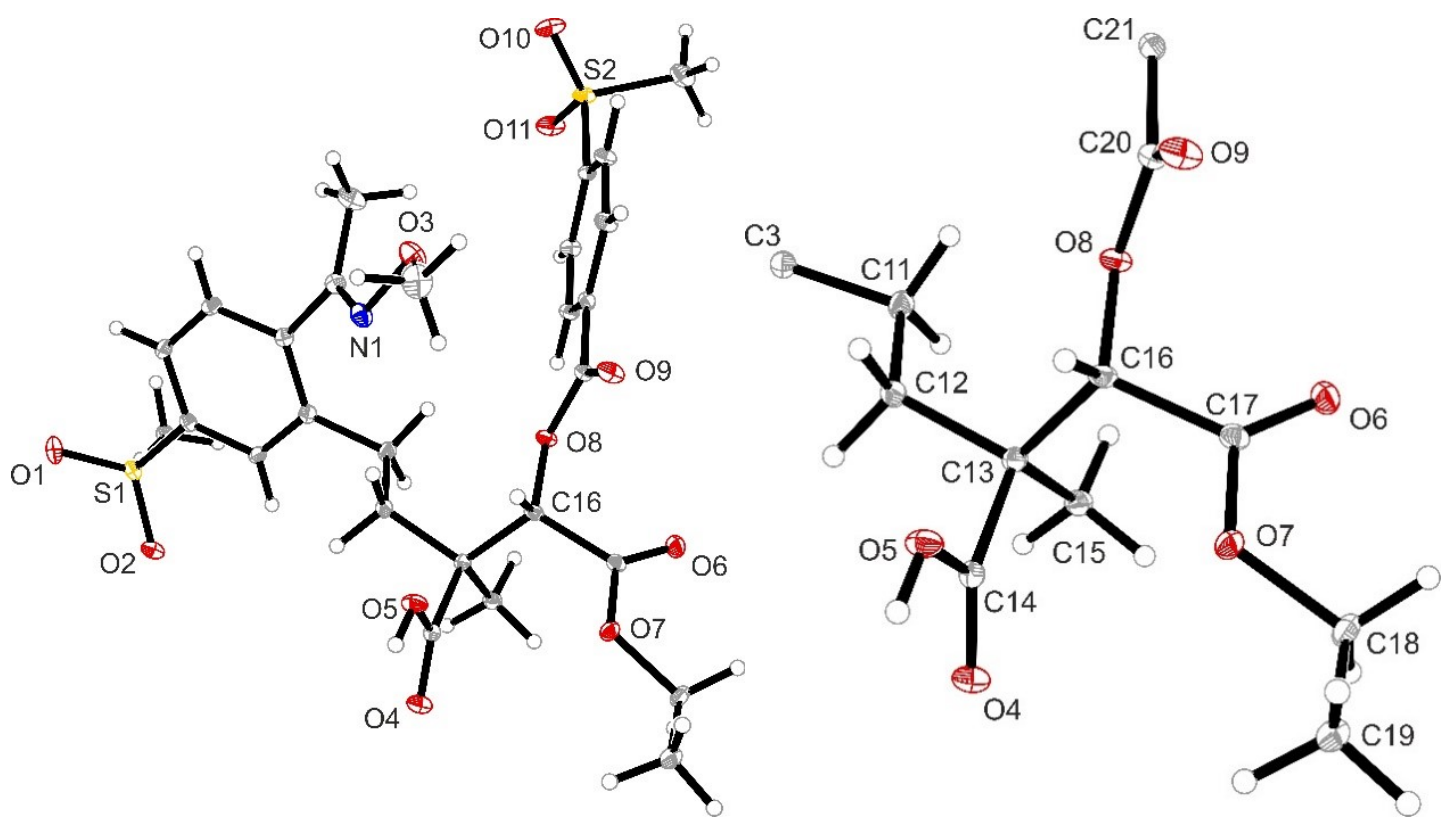

Figure S7. a) Crystal structure of compound S3. Thermal ellipsoids are shown at 30\% probability. b) Only the $C$-ipso of the aromatic ring systems are shown for clarity. 


\section{References}

1. APEX3 Version 2019.1-0, SAINT Version 8.40A and SADABS Bruker AXS area detector scaling and absorption correction Version 2016/2, Bruker AXS Inc., Madison, Wisconsin, USA, 2019.

2. Sheldrick, G. M., SHELXT - Integrated space-group and crystal-structure determination, Acta Cryst. 2015, A71, 3-8.

3. Sheldrick, G.M., Crystal structure refinement with SHELXL, Acta Cryst. 2015, C71 (1), 3-8.

4. XP-Interactive molecular graphics, Version 5.1, Bruker AXS Inc., Madison, Wisconsin, USA, 1998.

5. Too, P. C.; Wang, Y.-F.; Chiba, S. Org. Lett. 2010, 12, 5688-5691.

6. Silvi, M.; Aggarwal, V. K. J. Am. Chem. Soc. 2019, 141, 9511-9515.

7. Ociepa, M.; Wierzba, A. J.; Turkowska, J.; Gryko, D. J. Am. Chem. Soc. 2020, 142, 5355-5361.

8. Fawcett, A.; Murtaza, A.; Gregson, C. H. U.; Aggarwal, V. K. J. Am. Chem. Soc. 2019, 141, 4573-4578.

9. Kim, J. H.; Ruffoni, A.; Al-Faiyz, Y. S. S.; Sheikh, N. S.; Leonori, D. Angew. Chem. Int. Ed. 2020, 59, 8225-8231.

10. Proudfoot, J. R.; Betageri, R.; Cardozo, M.; Gilmore, T. A.; Glynn, S.; Hickey, E. R.; Jakes, S.; Kabcenell, A.; Kirrane, T. M.; Tibolla, A. K.; Lukas, S.; Patel, U. R.; Sharma, R.; Yazdanian, M.; Moss, N.; Beaulieu, P. L.; Cameron, D. R.; Ferland, J.-M.; Gauthier, J.; Gillard, J.; Gorys, V.; Poirier, M.; Rancourt, J.; Wernic, D.; Llinas-Brunet, M. J. Med. Chem. 2001, 44, 24212431.

11. Kawamura, S.; Unno, Y.; Asai, A.; Arisawa, M.; Shuto, S. Org. Biomol. Chem. 2013, 11, 66156622.

12. Belibel, R.; Barbaud, C. J. Polym. Sci., Part A: Polym. Chem. 2015, 53, 2586-2597.

13. Hall, H. K., Jr.; Padias, A. B. J. Polym. Sci., Part A: Polym. Chem. 2003, 41, 625-635.

14. Jakob, U.; Mundinger, S.; Bannwarth, W. Eur. J. Org. Chem. 2014, 6963-6974. 
8. NMR Spectra

|

$\stackrel{\circ}{\circ}$

กี
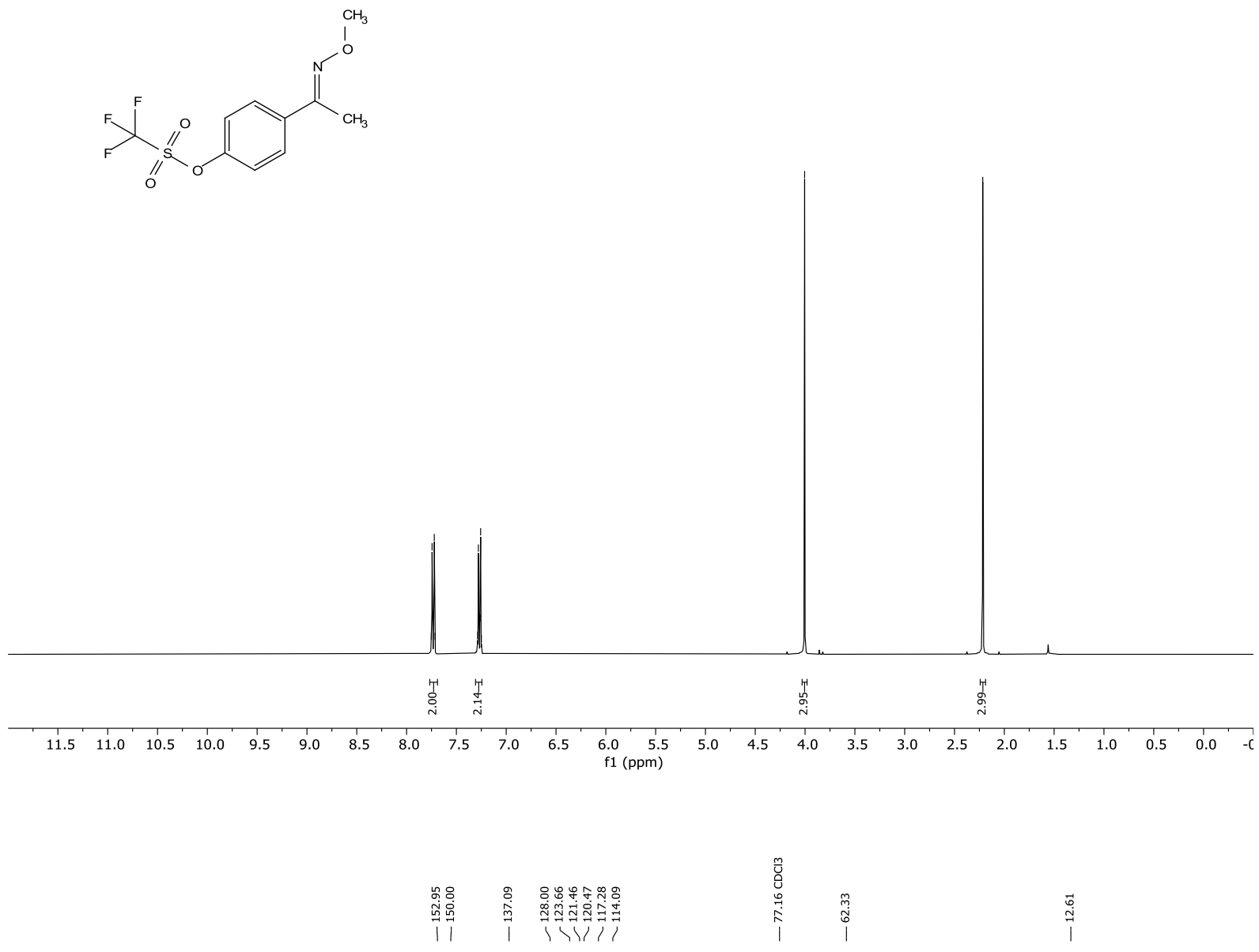

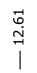
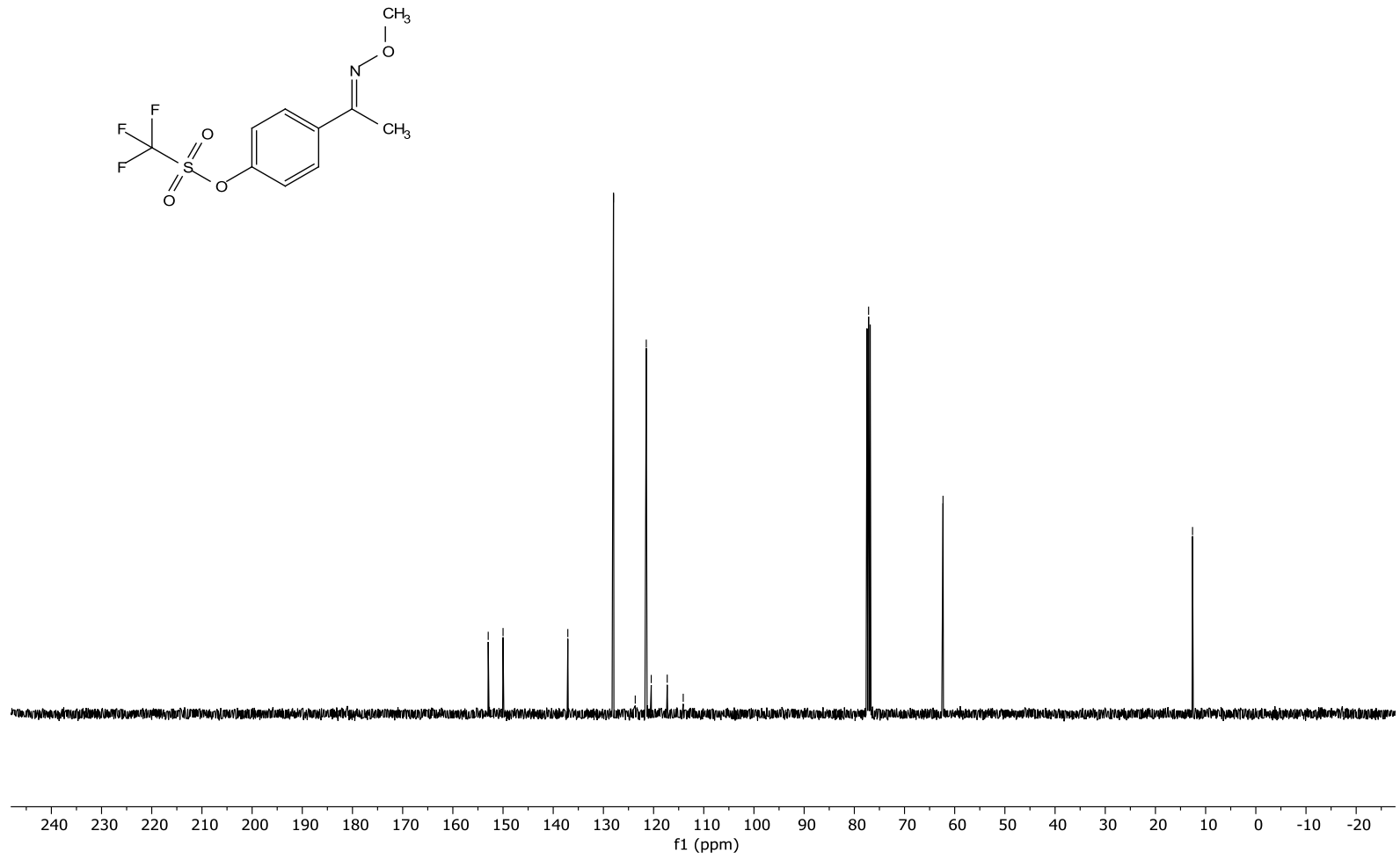

S43 


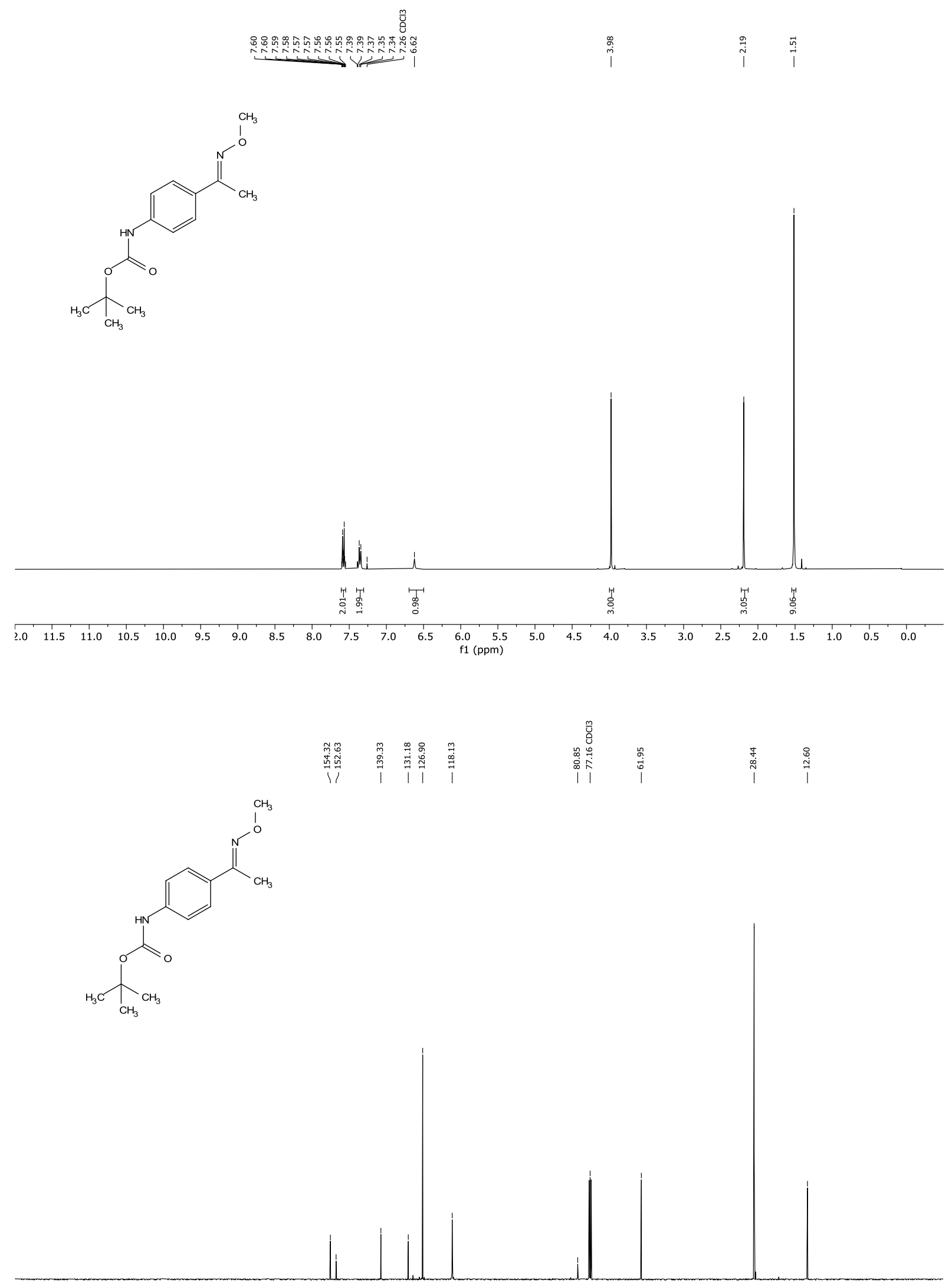

$\begin{array}{llllllllllllllllllllllllllllllll}1 & 1 \\ 240 & 230 & 220 & 210 & 200 & 190 & 180 & 170 & 160 & 150 & 140 & 130 & 120 & 110 & 100 & 90 & 80 & 70 & 60 & 50 & 40 & 30 & 20 & 10 & 0 & -10 & -20\end{array}$ 
荅

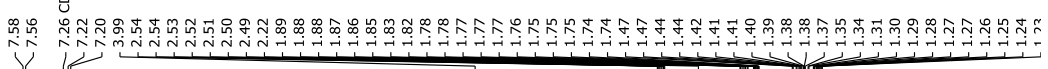
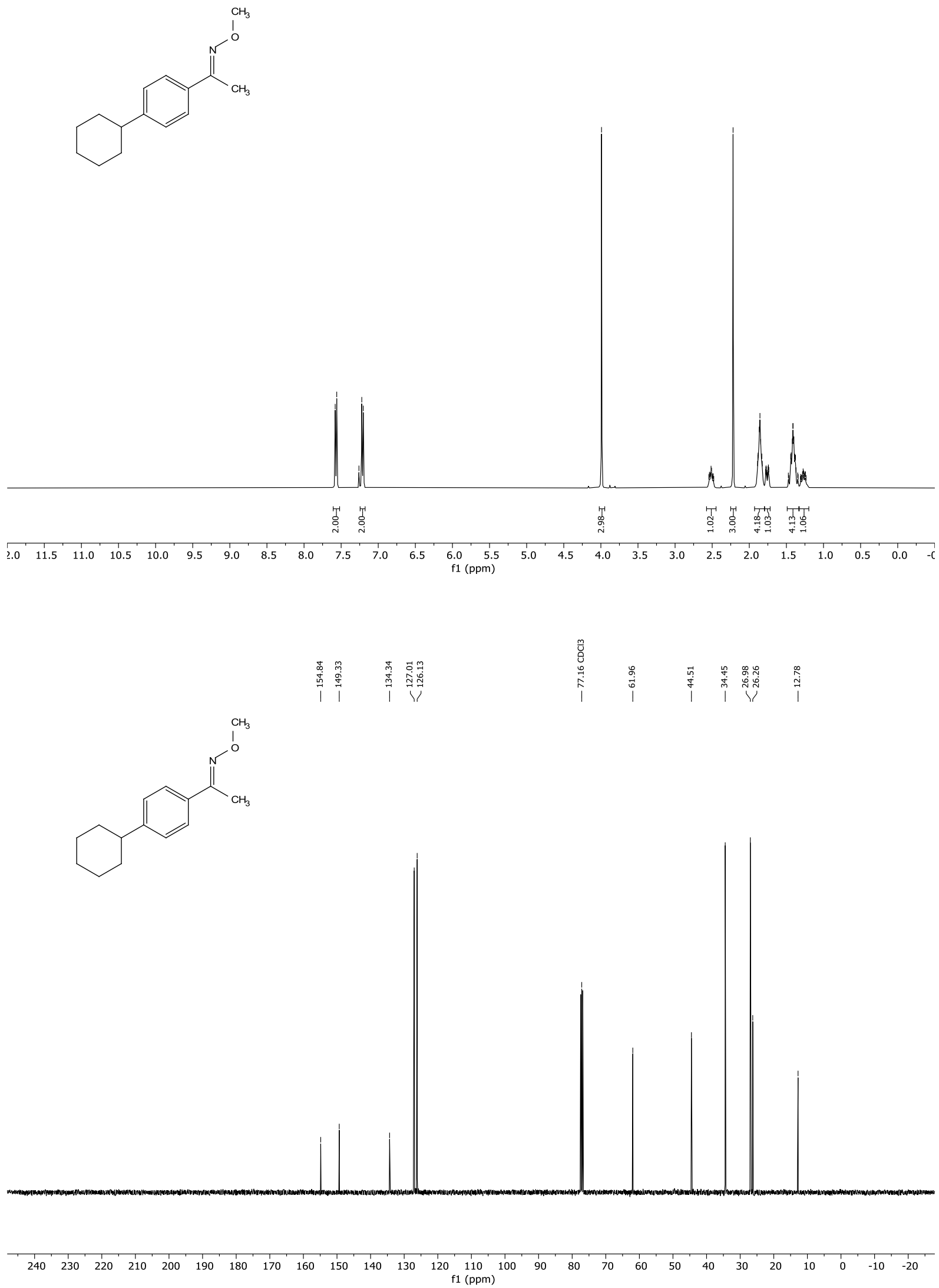

S45 


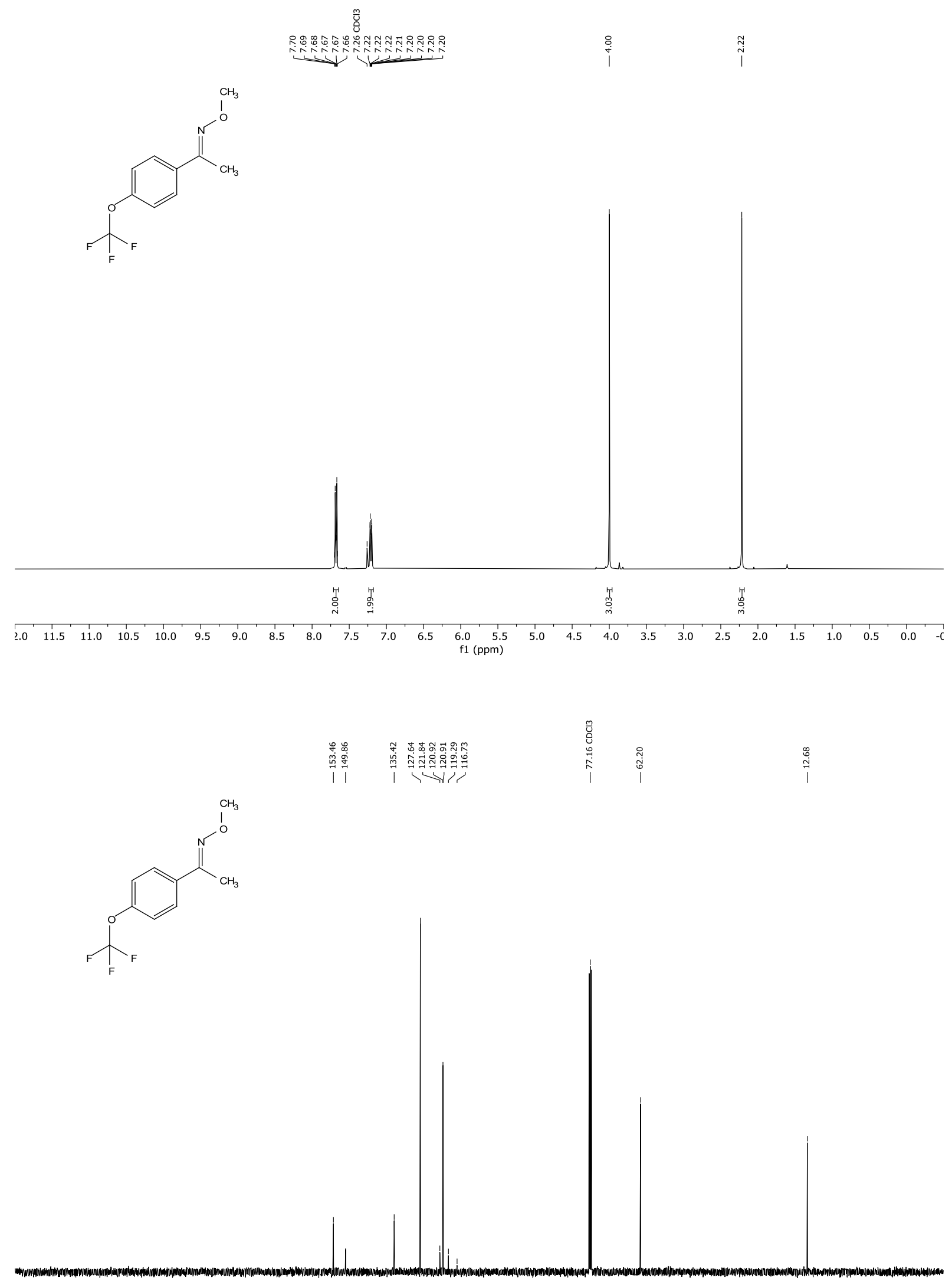

$\begin{array}{rlllllllllllllllllllllllllllllllll}140 & 230 & 220 & 210 & 200 & 190 & 180 & 170 & 160 & 150 & 140 & 130 & 120 & 110 & 100 & 90 & 80 & 70 & 60 & 50 & 40 & 30 & 20 & 10 & 0 & -10 & -20\end{array}$ 


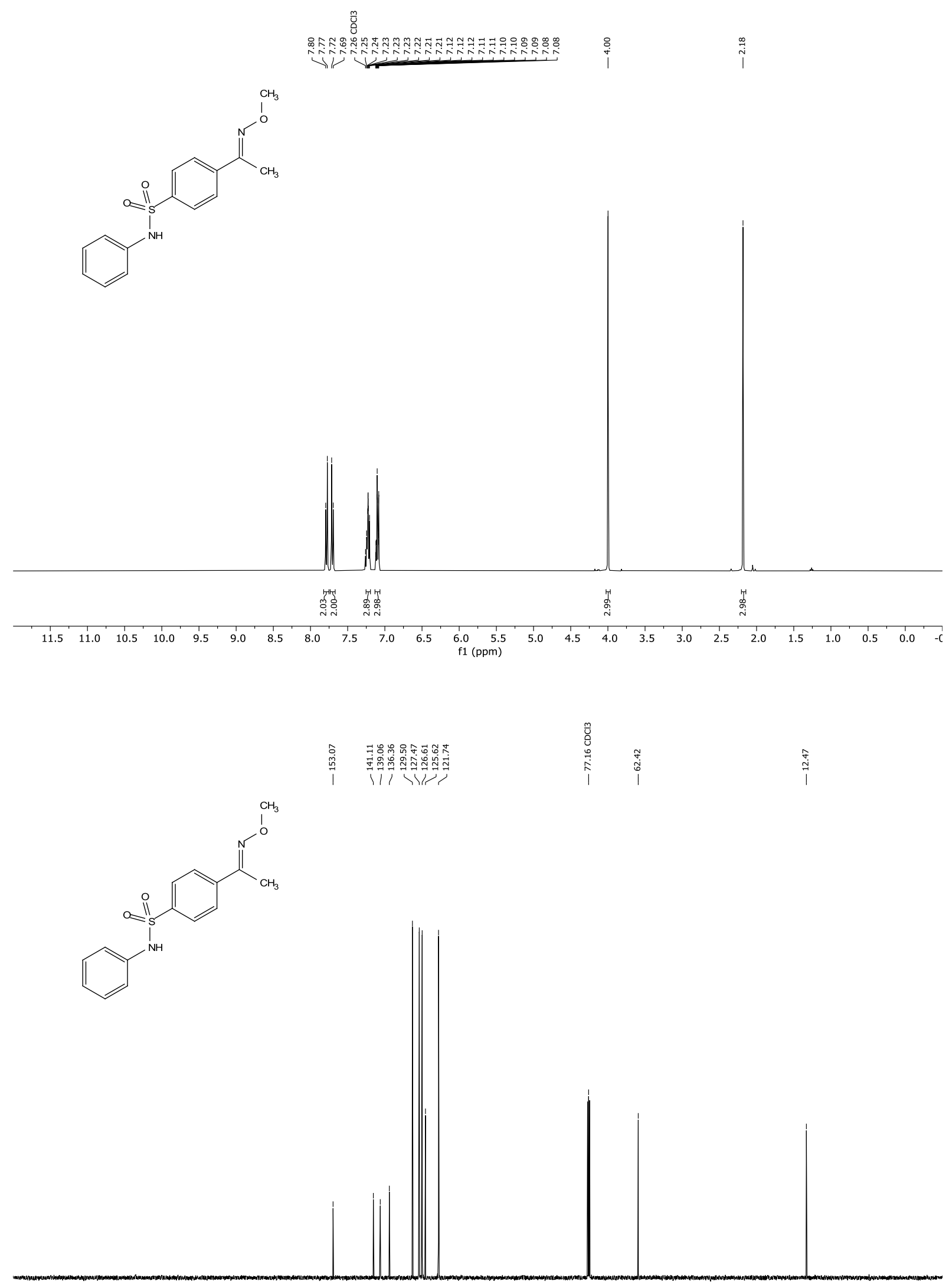

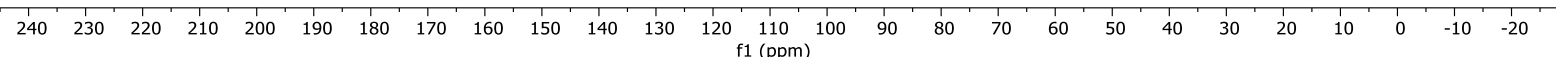




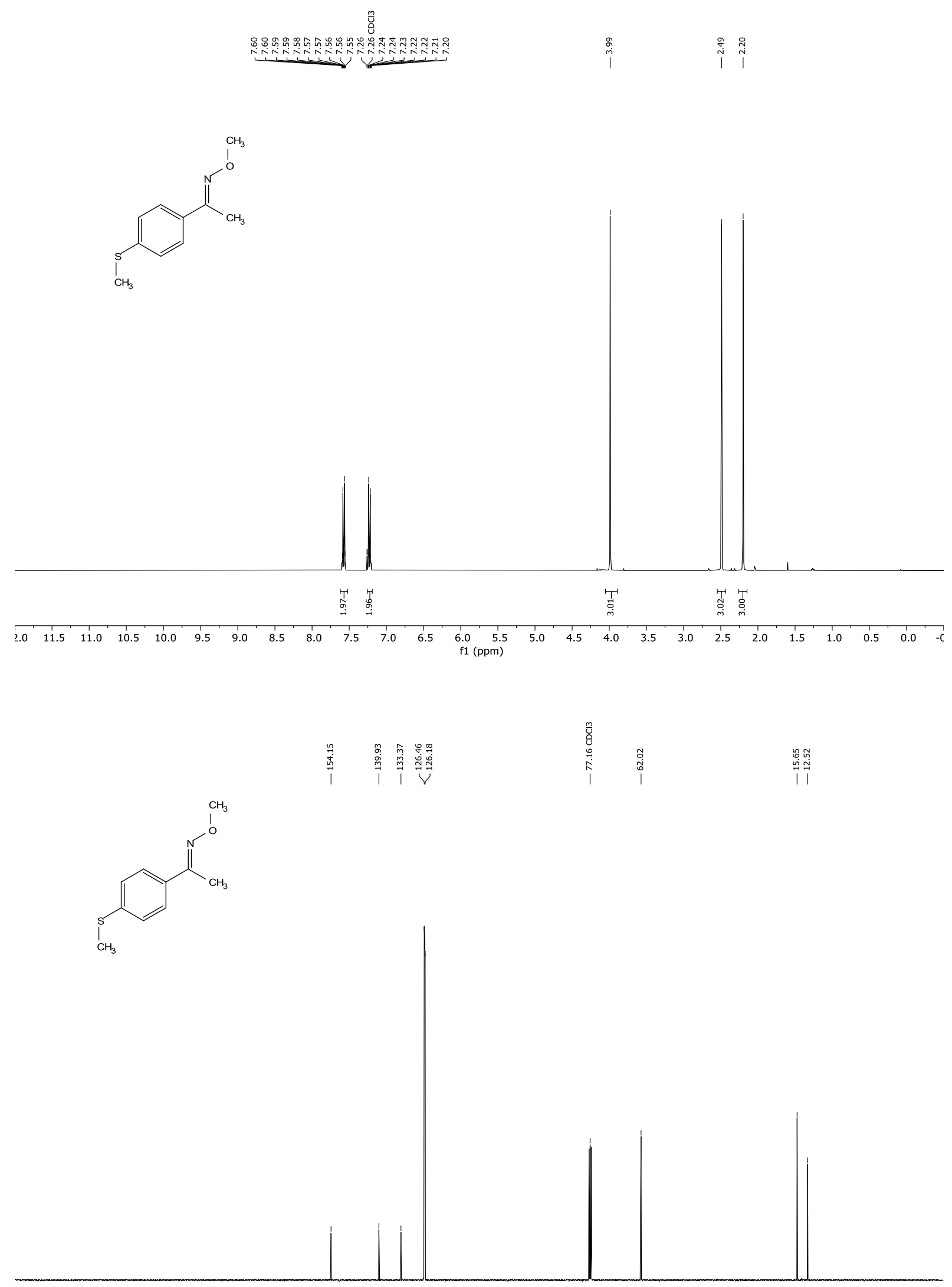

$\begin{array}{llllllllllllllllllllllllllllllll}1 & 240 & 230 & 220 & 210 & 200 & 190 & 180 & 170 & 160 & 150 & 140 & 130 & 120 & 110 & 100 & 90 & 80 & 70 & 60 & 50 & 40 & 30 & 20 & 10 & 0 & -10 & -20\end{array}$ 


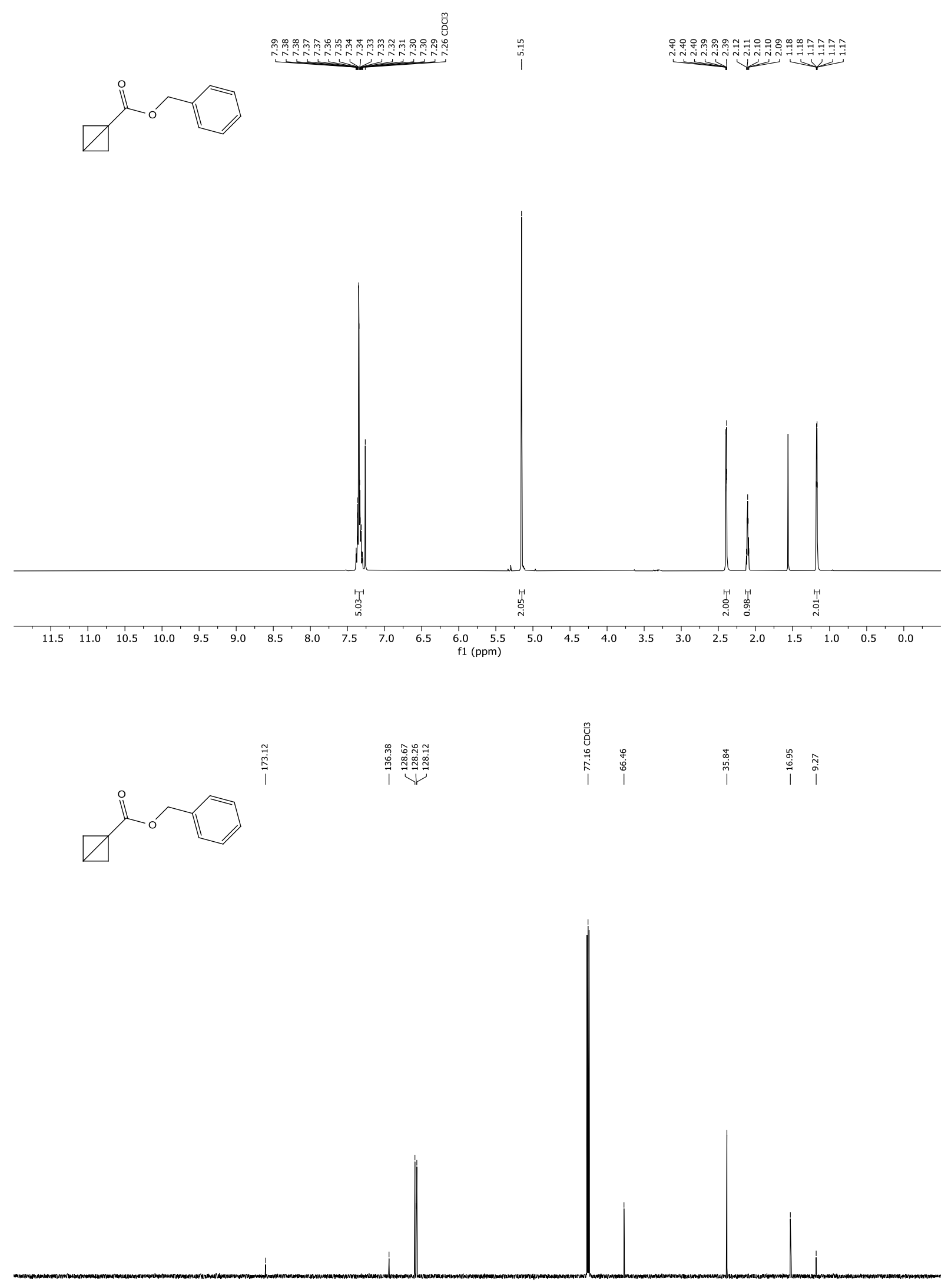

$\begin{array}{lllllllllllllllllllllllllllll}1 & 1 \\ 240 & 230 & 220 & 210 & 200 & 190 & 180 & 170 & 160 & 150 & 140 & 130 & 120 & 110 & 100 & 90 & 80 & 70 & 60 & 50 & 40 & 30 & 20 & 10 & 0 & -10 & -20\end{array}$ 


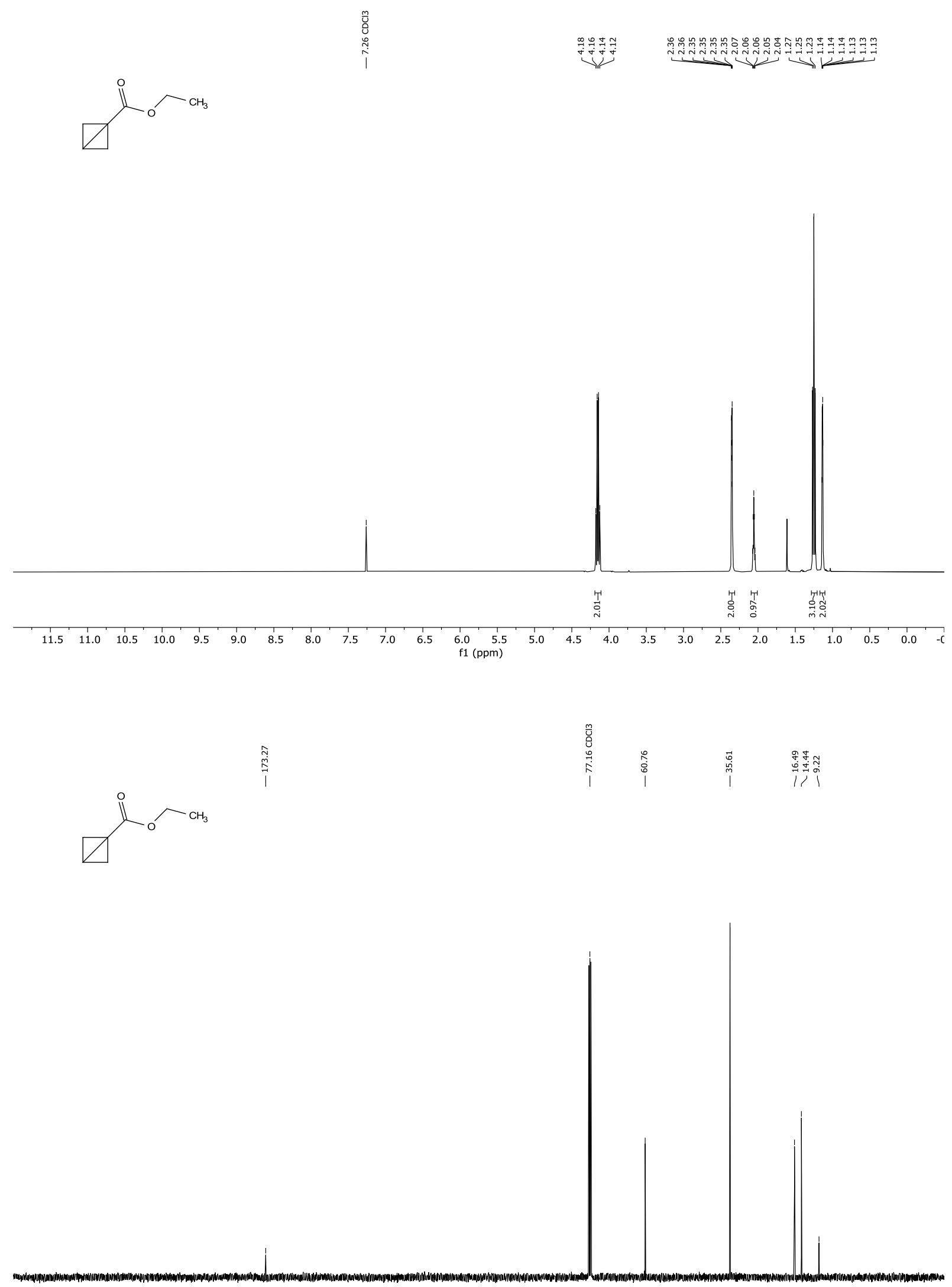

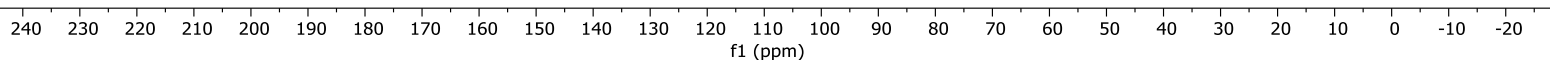


<smiles>CC(C)COC(=O)C1CC1</smiles>

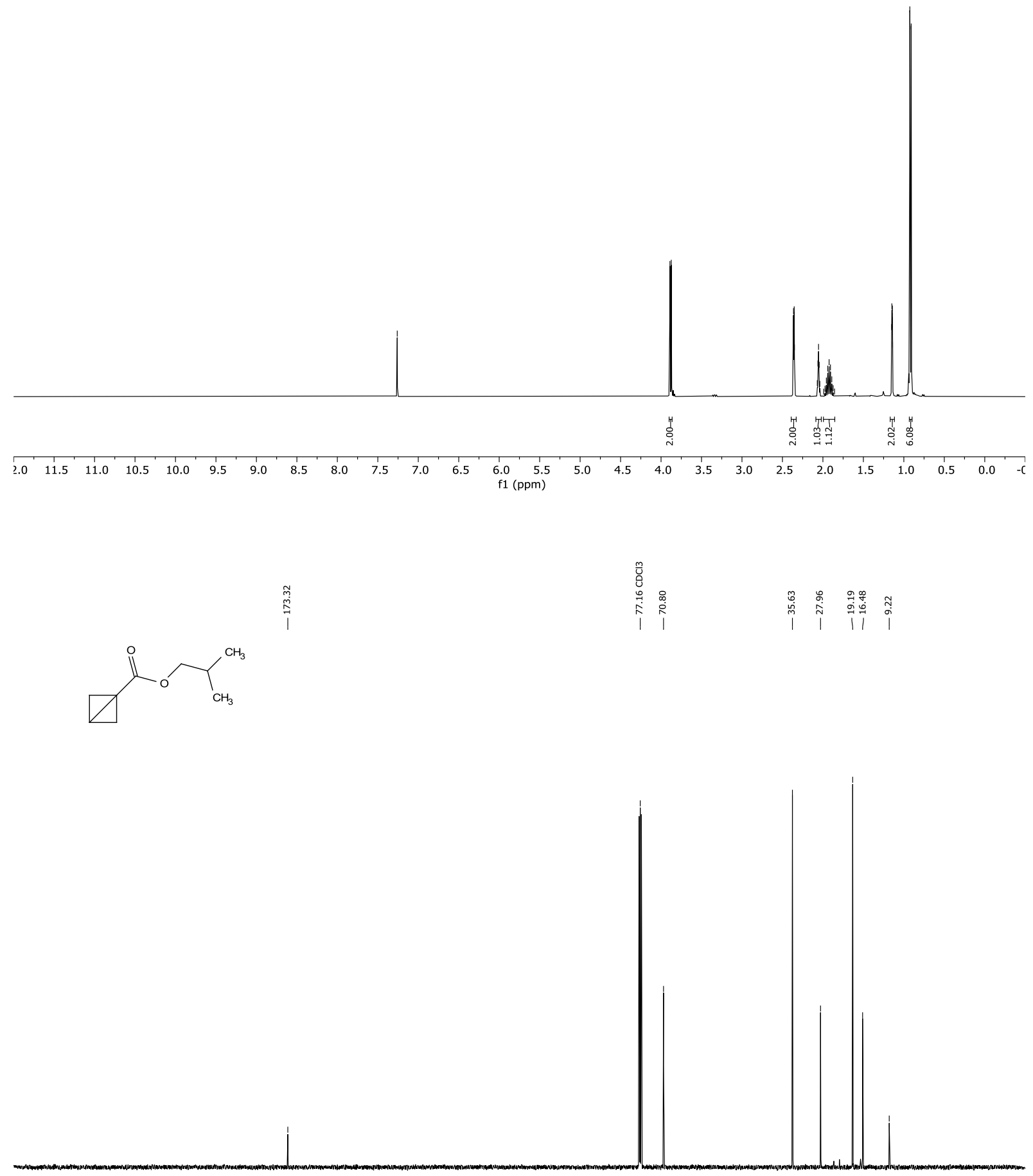

$\begin{array}{lllllllllllllllllllllllllllllllll}1 & 240 & 230 & 220 & 210 & 200 & 190 & 180 & 170 & 160 & 150 & 140 & 130 & 120 & 110 & 100 & 90 & 80 & 70 & 60 & 50 & 40 & 30 & 20 & 10 & 0 & -10 & -20\end{array}$ 


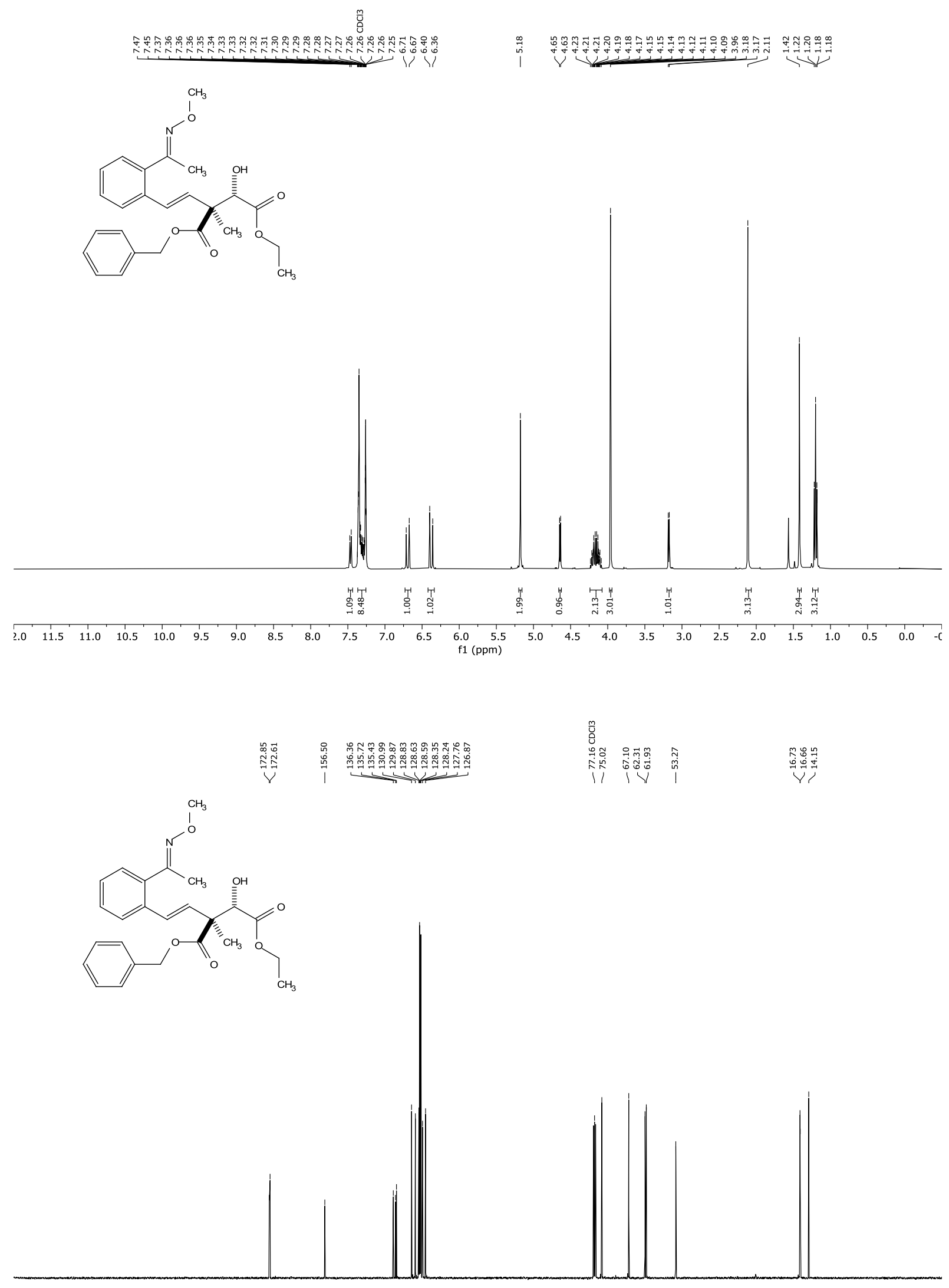

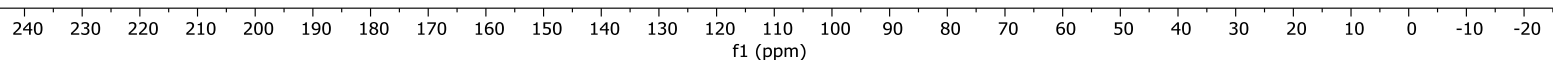




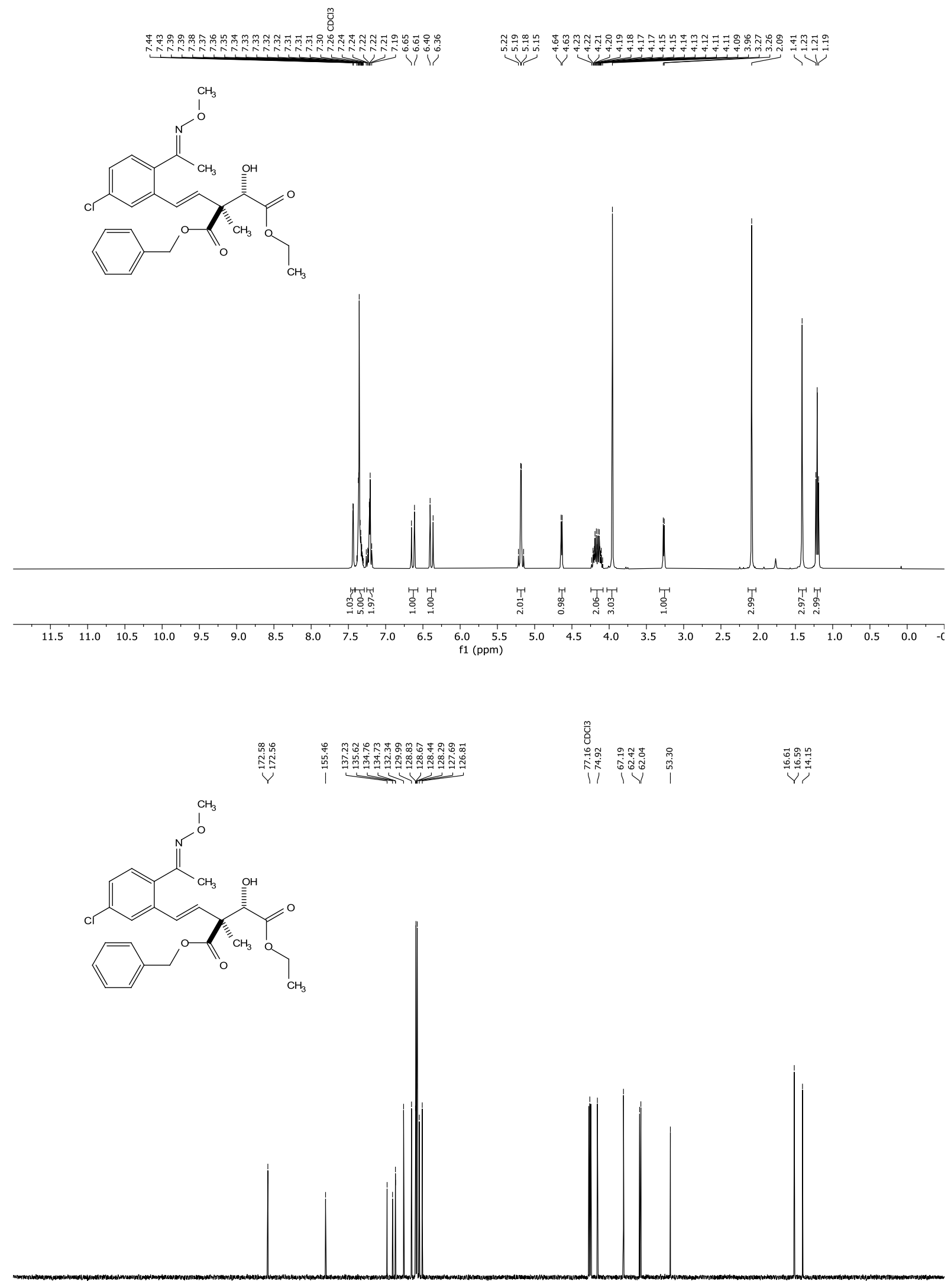

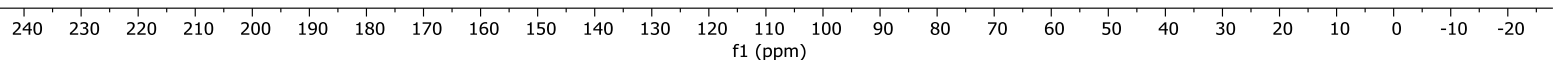




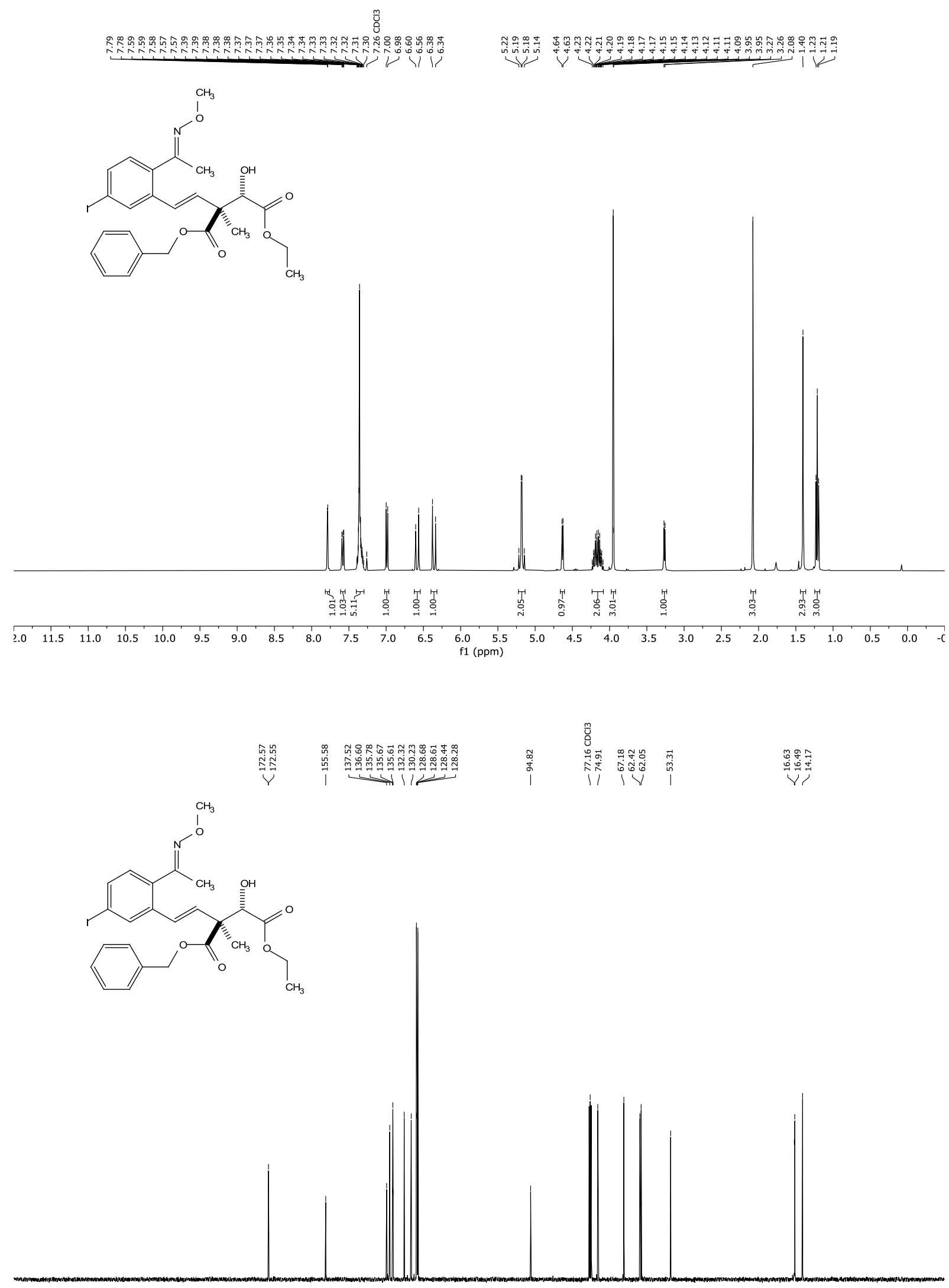

$\begin{array}{rllllllllllllllllllllllllllll}1 & 240 & 230 & 220 & 210 & 200 & 190 & 180 & 170 & 160 & 150 & 140 & 130 & 120 & 110 & 100 & 90 & 80 & 70 & 60 & 50 & 40 & 30 & 20 & 10 & 0 & -10 & -20\end{array}$ 


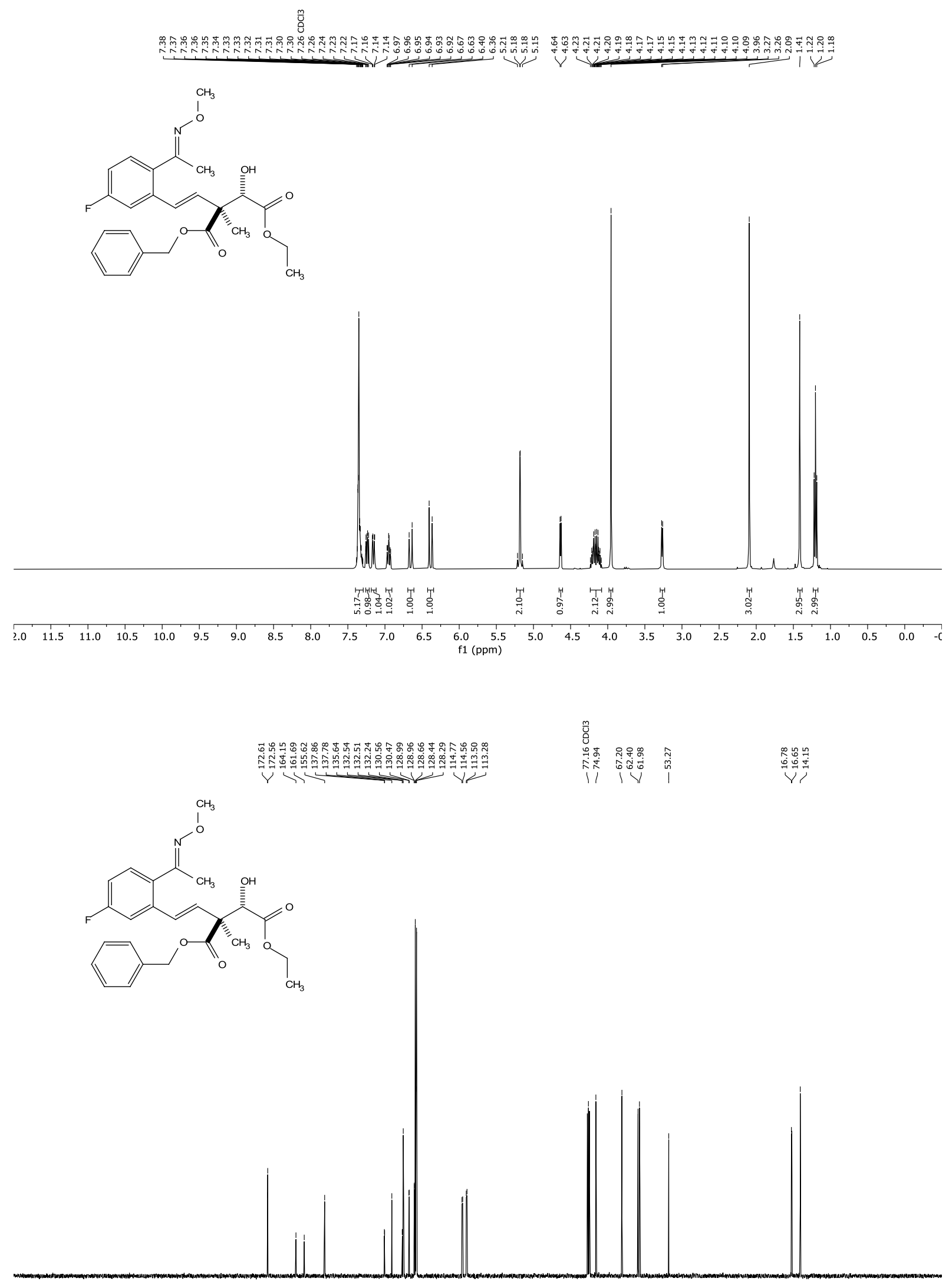

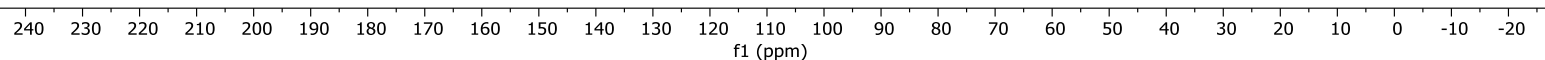




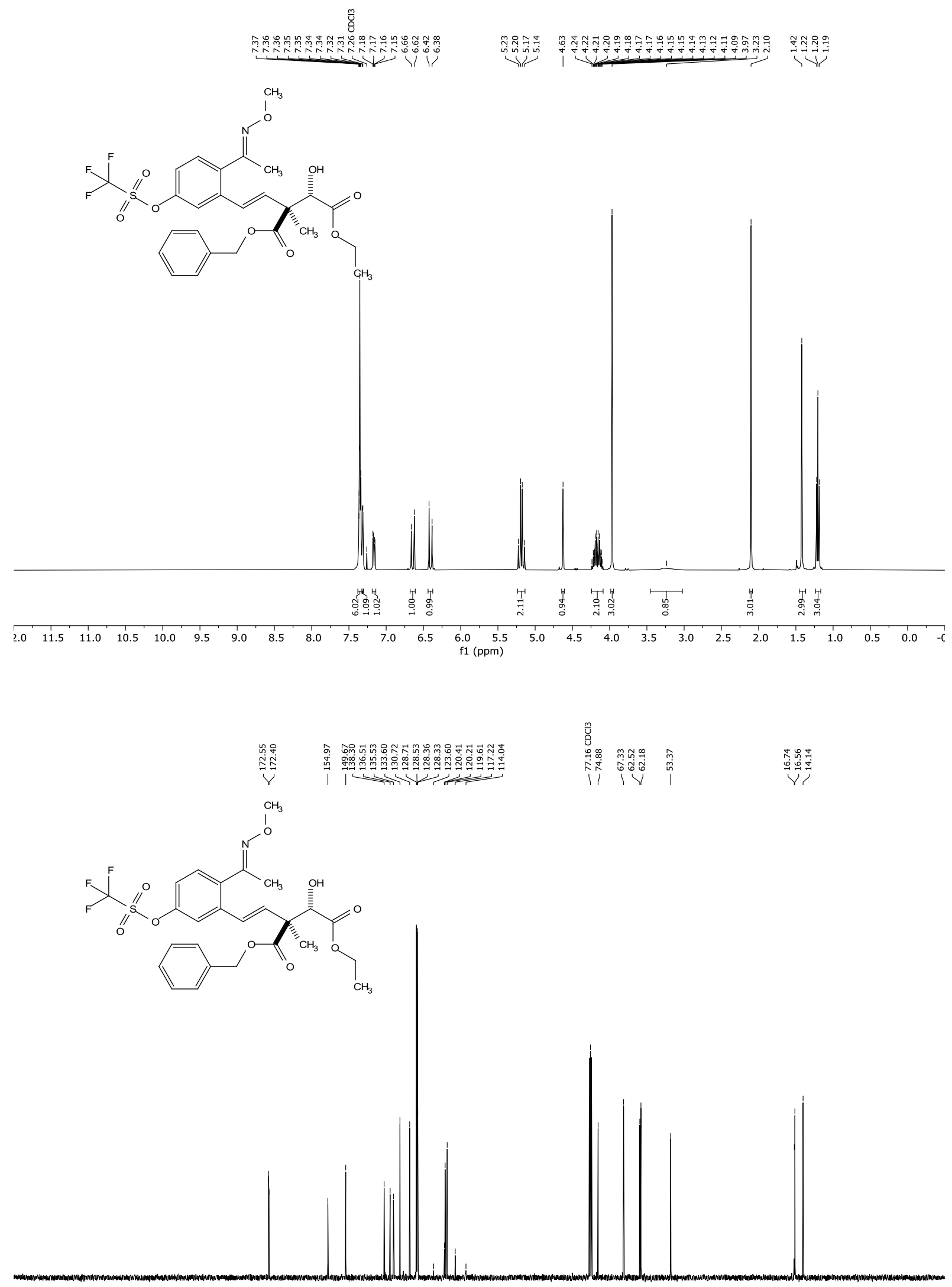

$\begin{array}{llllllllllllllllllllllllllllllll}1 & 1 \\ 240 & 230 & 220 & 210 & 200 & 190 & 180 & 170 & 160 & 150 & 140 & 130 & 120 & 110 & 100 & 90 & 80 & 70 & 60 & 50 & 40 & 30 & 20 & 10 & 0 & -10 & -20\end{array}$ 

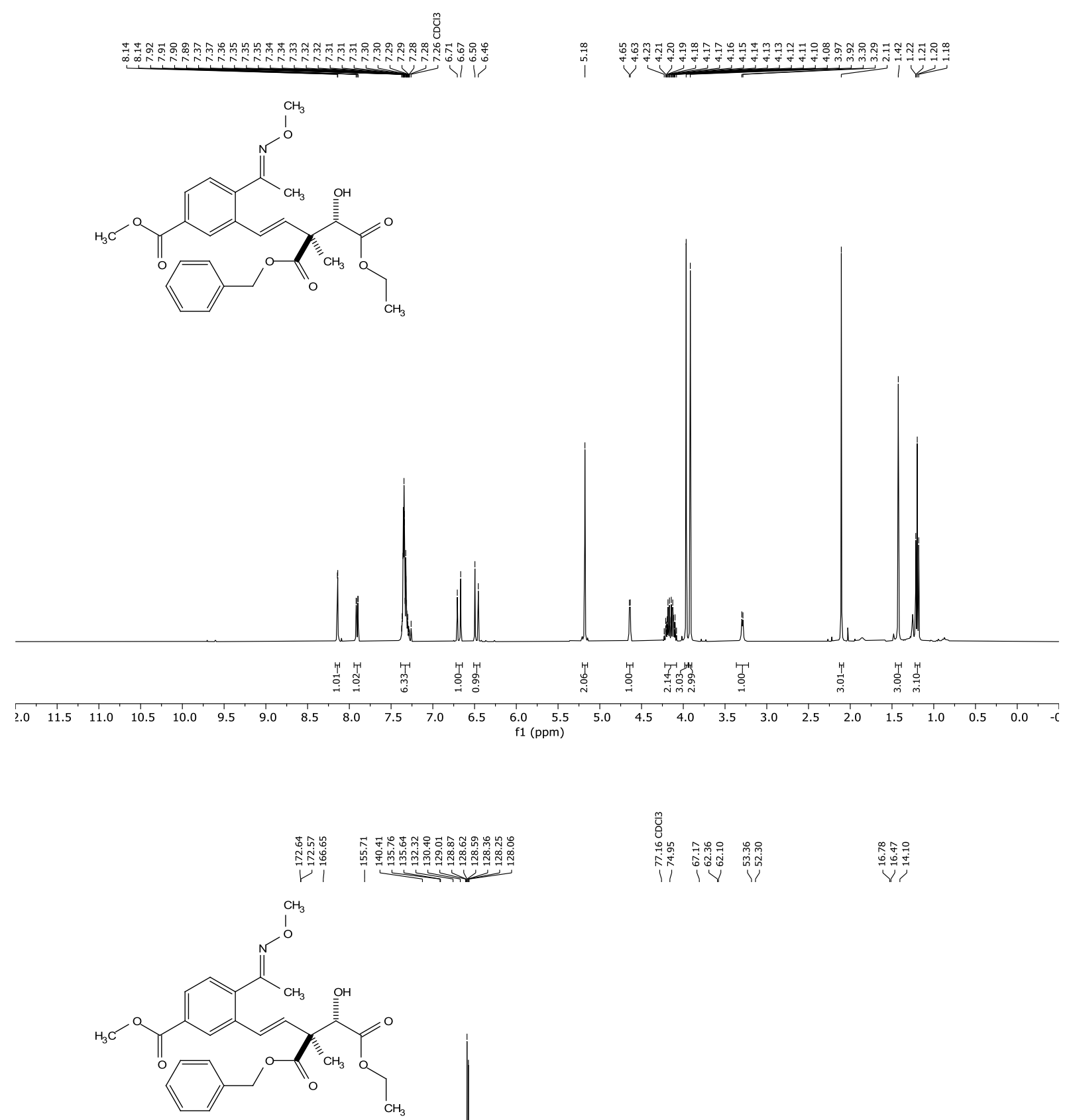

$\begin{array}{lllllllllllllllllllllllllll}240 & 230 & 220 & 210 & 200 & 190 & 180 & 170 & 160 & 150 & 140 & 130 & 120 & 110 & 100 & 90 & 80 & 70 & 60 & 50 & 40 & 30 & 20 & 10 & 0 & -10 & -20\end{array}$ 


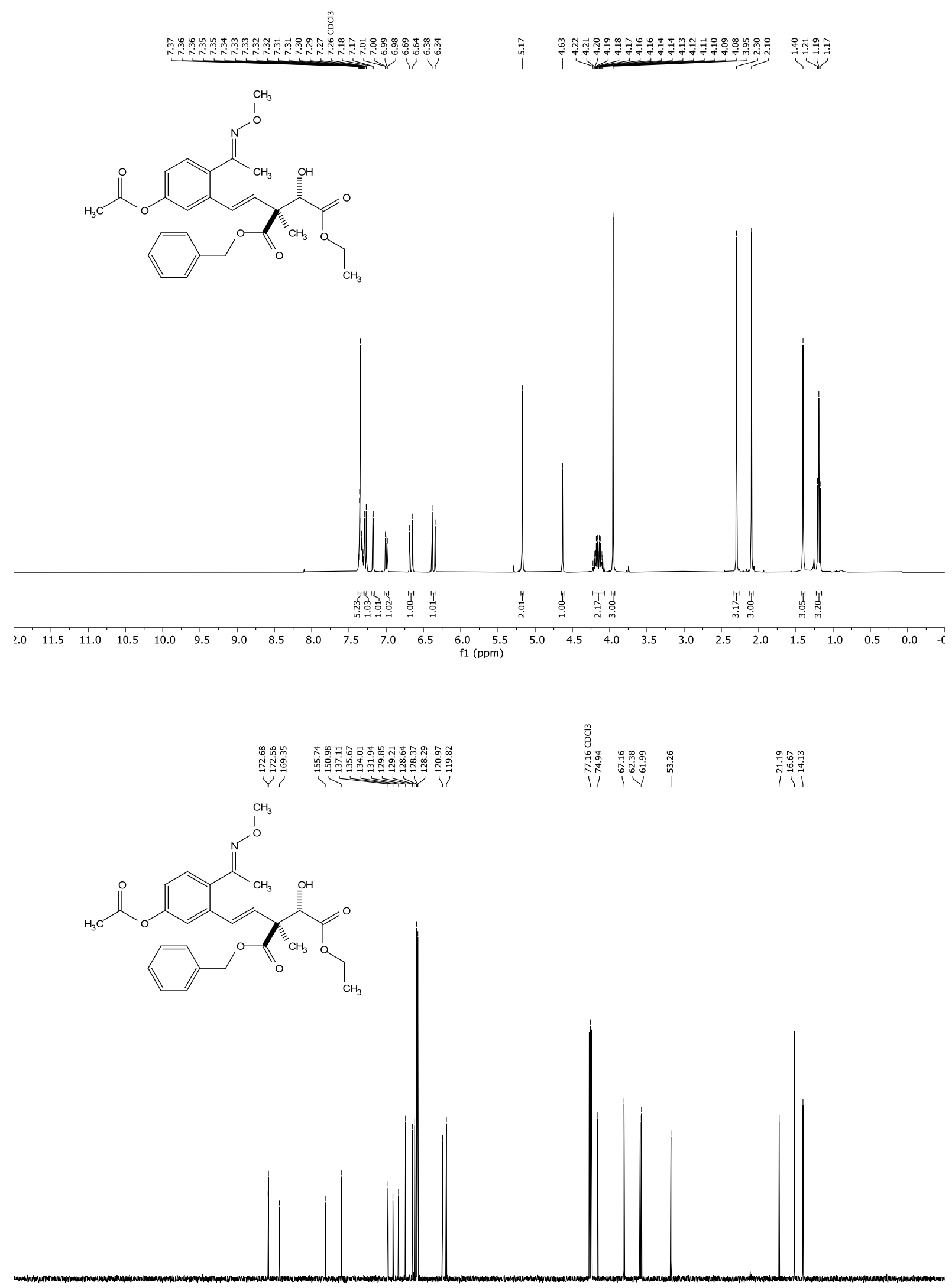

$\begin{array}{rlllllllllllllllllllllllllllll}1 & 240 & 230 & 220 & 210 & 200 & 190 & 180 & 170 & 160 & 150 & 140 & 130 & 120 & 110 & 100 & 90 & 80 & 70 & 60 & 50 & 40 & 30 & 20 & 10 & 0 & -10 & -20\end{array}$ 


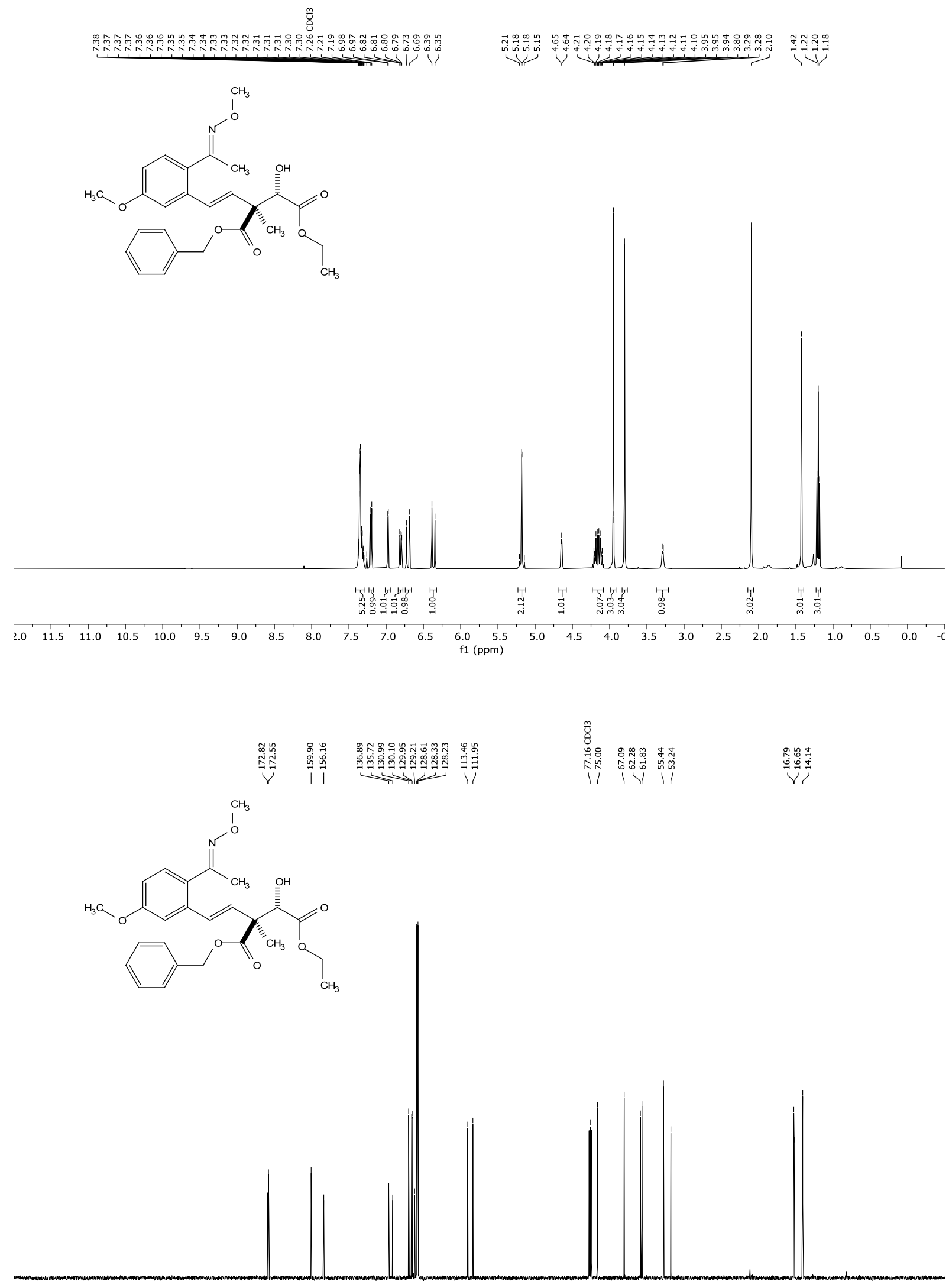

$\begin{array}{lllllllllllllllllllllllllllllll}1 & 240 & 230 & 220 & 210 & 200 & 190 & 180 & 170 & 160 & 150 & 140 & 130 & 120 & 110 & 100 & 90 & 80 & 70 & 60 & 50 & 40 & 30 & 20 & 10 & 0 & -10 & -20\end{array}$ 


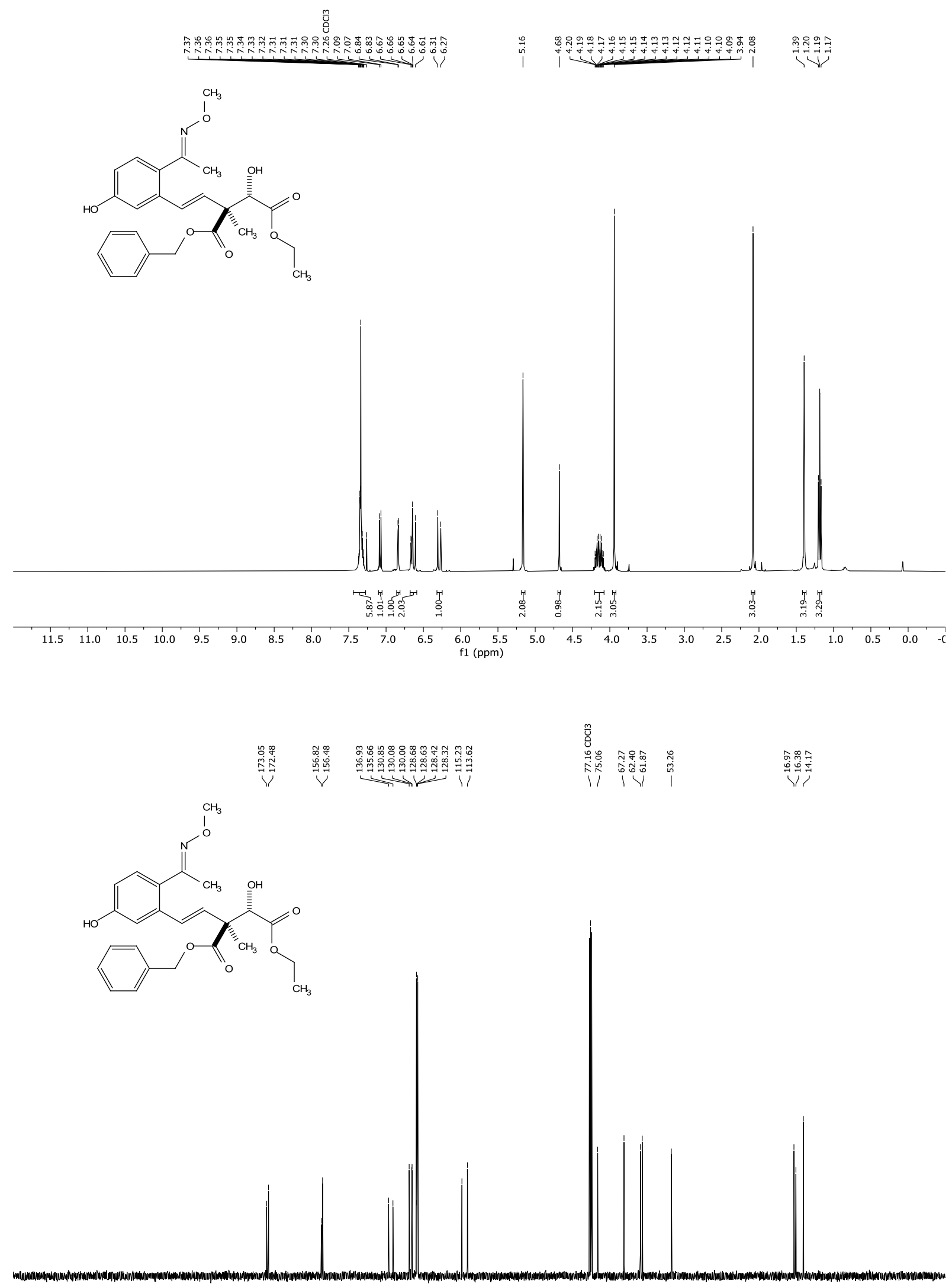

$\begin{array}{lllllllllllllllllllllllllllllllllll}240 & 230 & 220 & 210 & 200 & 190 & 180 & 170 & 160 & 150 & 140 & 130 & 120 & 110 & 100 & 90 & 80 & 70 & 60 & 50 & 40 & 30 & 20 & 10 & 0 & -10 & -20\end{array}$ 

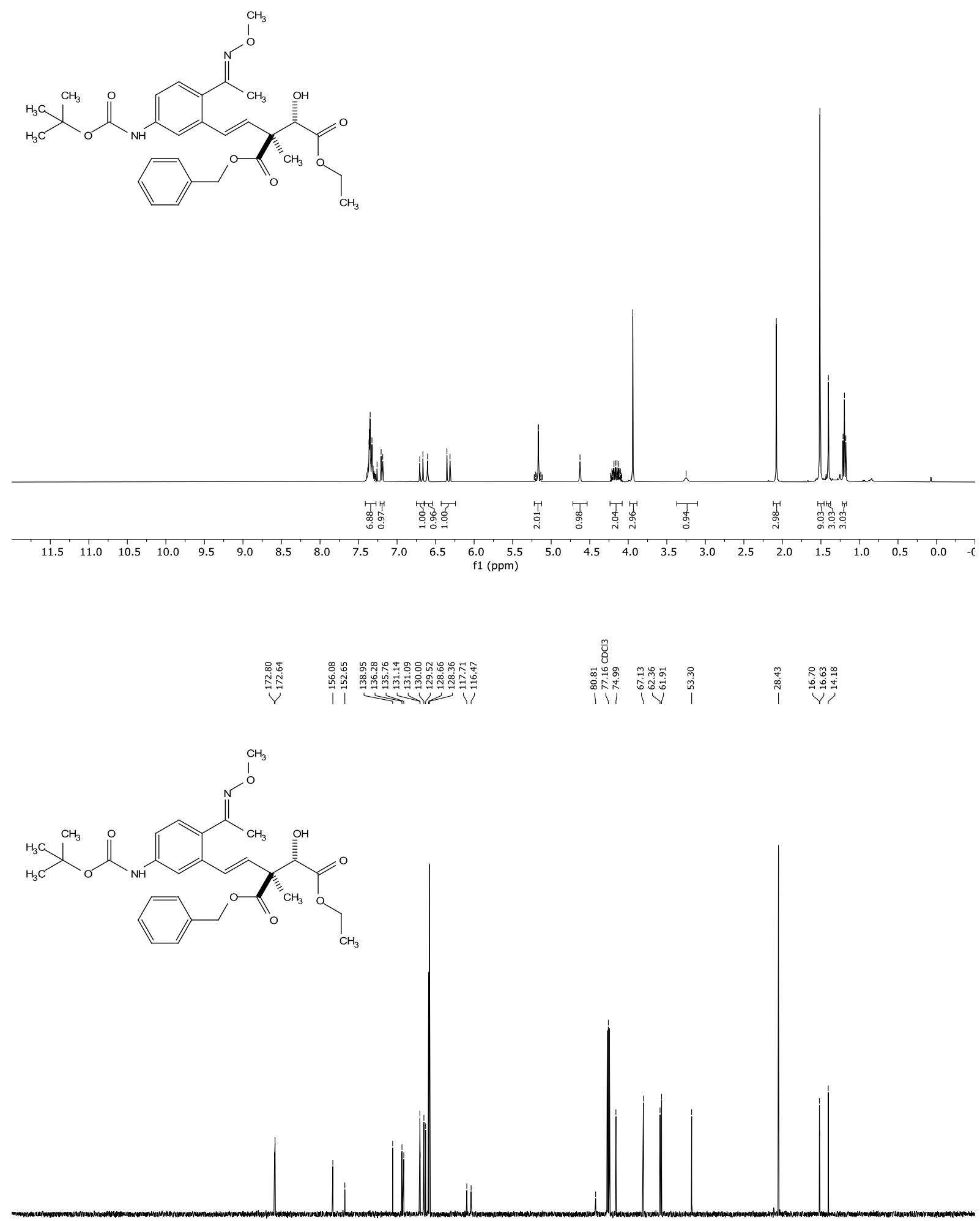

$\begin{array}{llllllllllllllllllllllllllllll}1 & 1 \\ 240 & 230 & 220 & 210 & 200 & 190 & 180 & 170 & 160 & 150 & 140 & 130 & 120 & 110 & 100 & 90 & 80 & 70 & 60 & 50 & 40 & 30 & 20 & 10 & 0 & -10 & -20\end{array}$ 
范

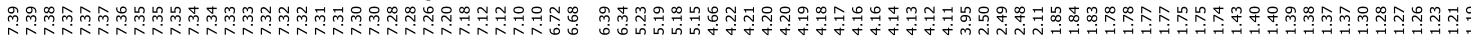<smiles>CCOC(=O)[C@H](O)[C@@](C)(/C=C/c1cc(C2CCCCC2)ccc1/C=N/OC)C(=O)OCc1ccccc1</smiles>
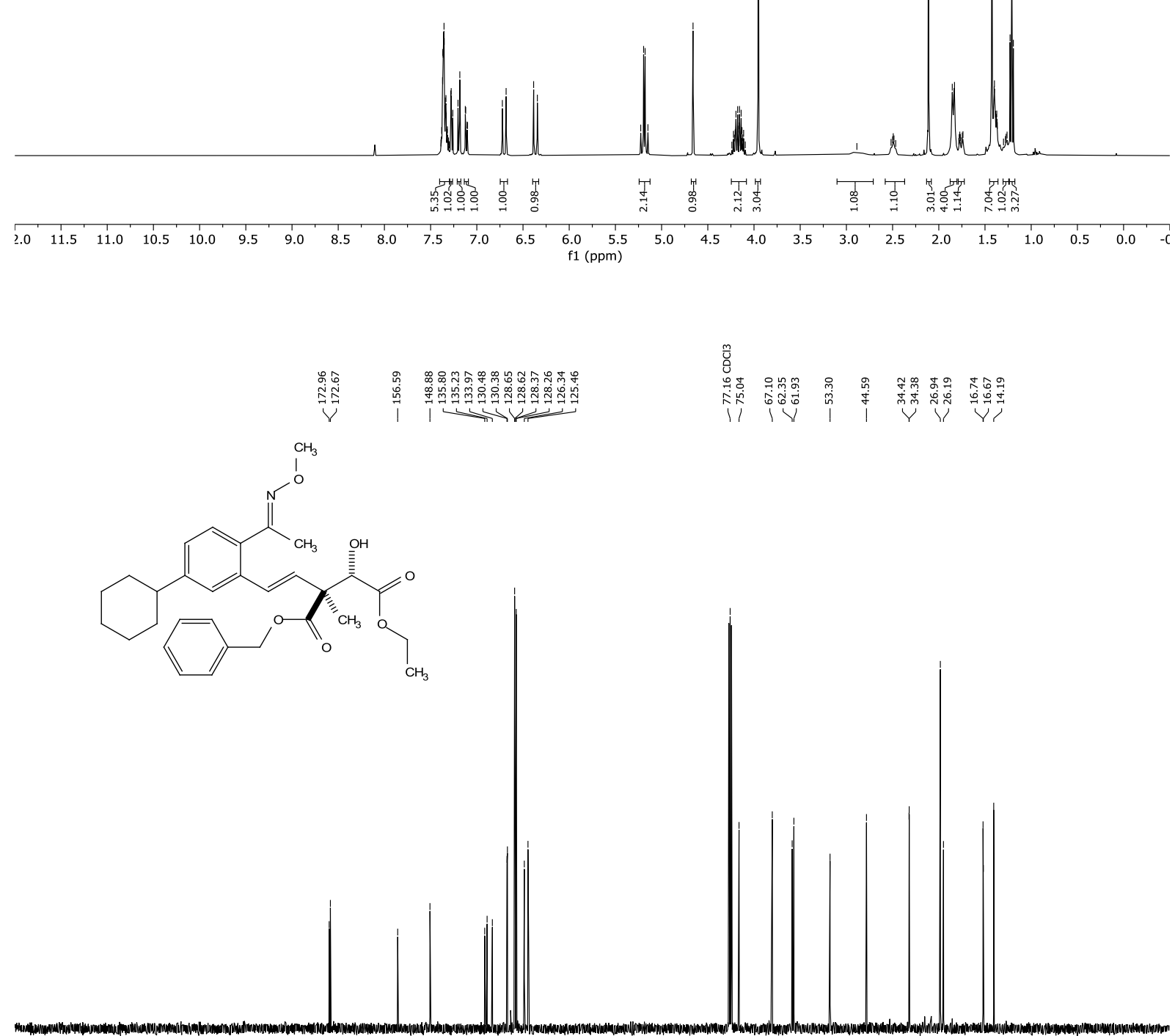

$\begin{array}{lllllllllllllllllllllllllllllllllllll}1 & 240 & 230 & 220 & 210 & 200 & 190 & 180 & 170 & 160 & 150 & 140 & 130 & 120 & 110 & 100 & 90 & 80 & 70 & 60 & 50 & 40 & 30 & 20 & 10 & 0 & -10 & -20\end{array}$ 
<smiles>CCOC(=O)[C@H](C)[C@@](C)(/C=C/c1cc(-c2ccccc2)ccc1/C=N/OC)C(=O)OCc1ccccc1</smiles>
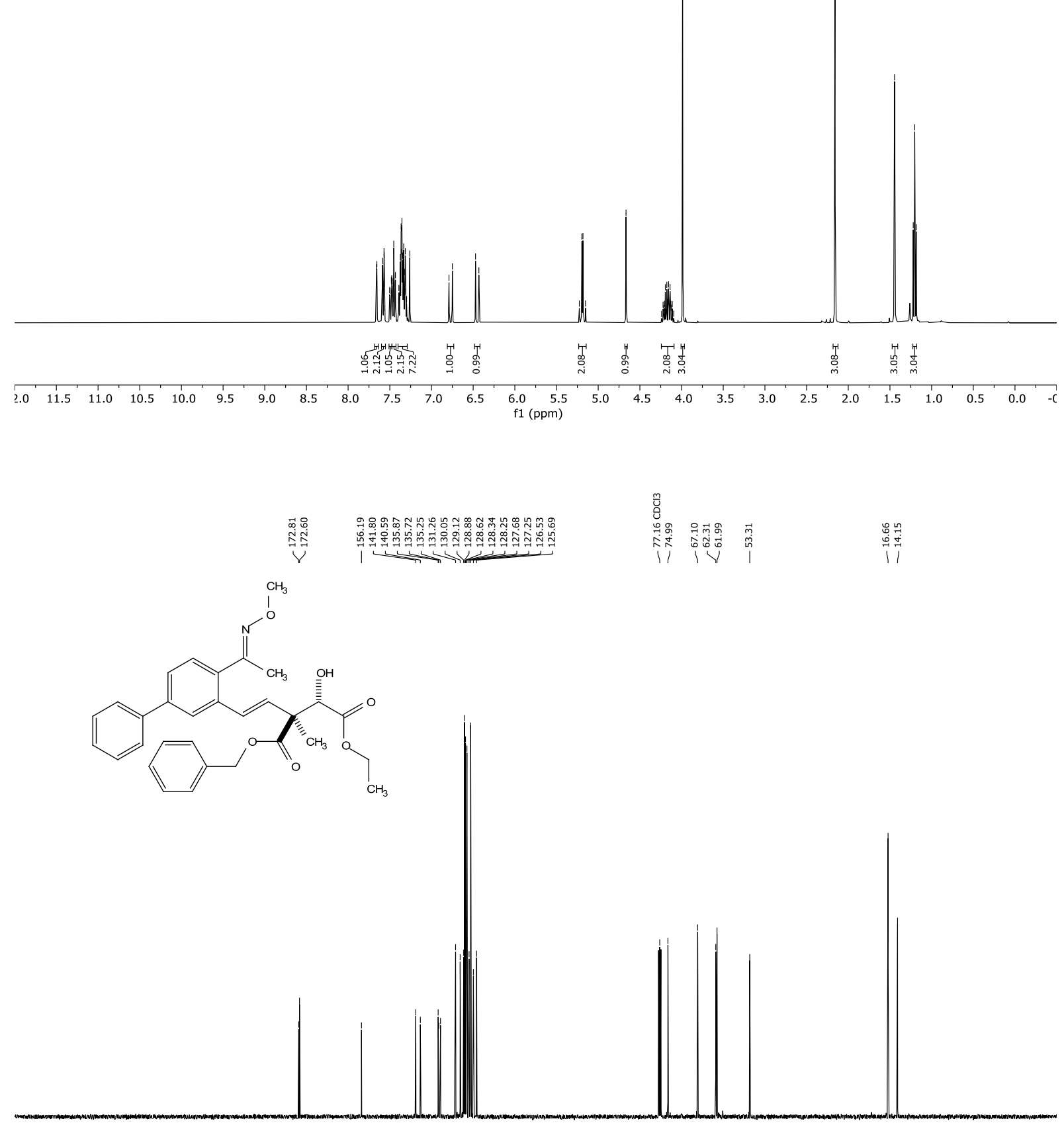

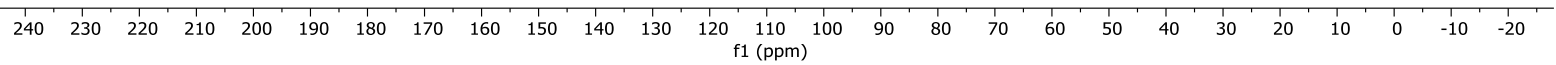


<smiles>CO/N=C/c1cc2ccccc2cc1/C=C/[C@@](C)(C(=O)OCc1ccccc1)[C@@H](O)C(=O)OCO</smiles>
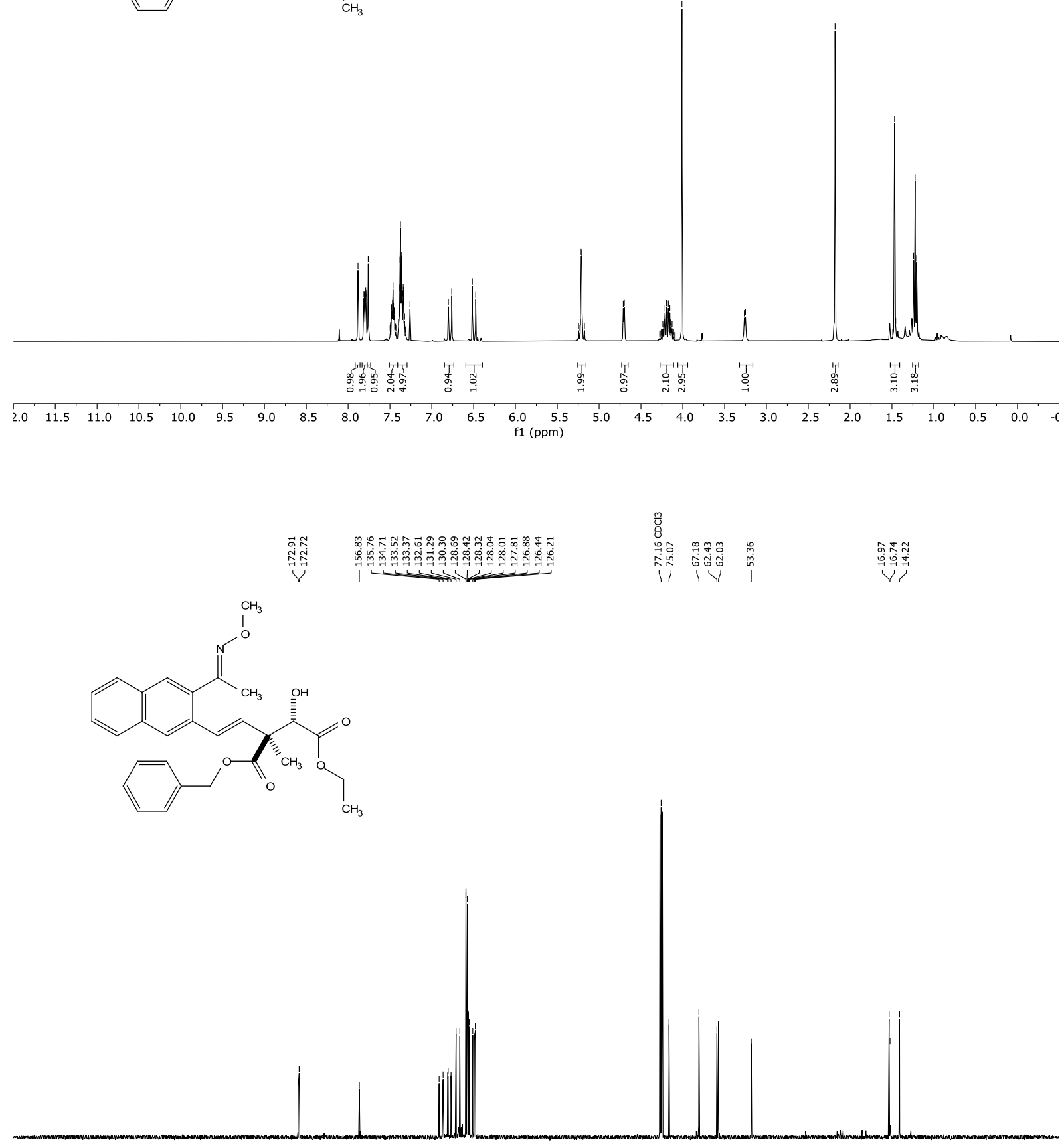

$\begin{array}{lllllllllllllllllllllllllllll}1 & 240 & 230 & 220 & 210 & 200 & 190 & 180 & 170 & 160 & 150 & 140 & 130 & 120 & 110 & 100 & 90 & 80 & 70 & 60 & 50 & 40 & 30 & 20 & 10 & 0 & -10 & -20\end{array}$ 


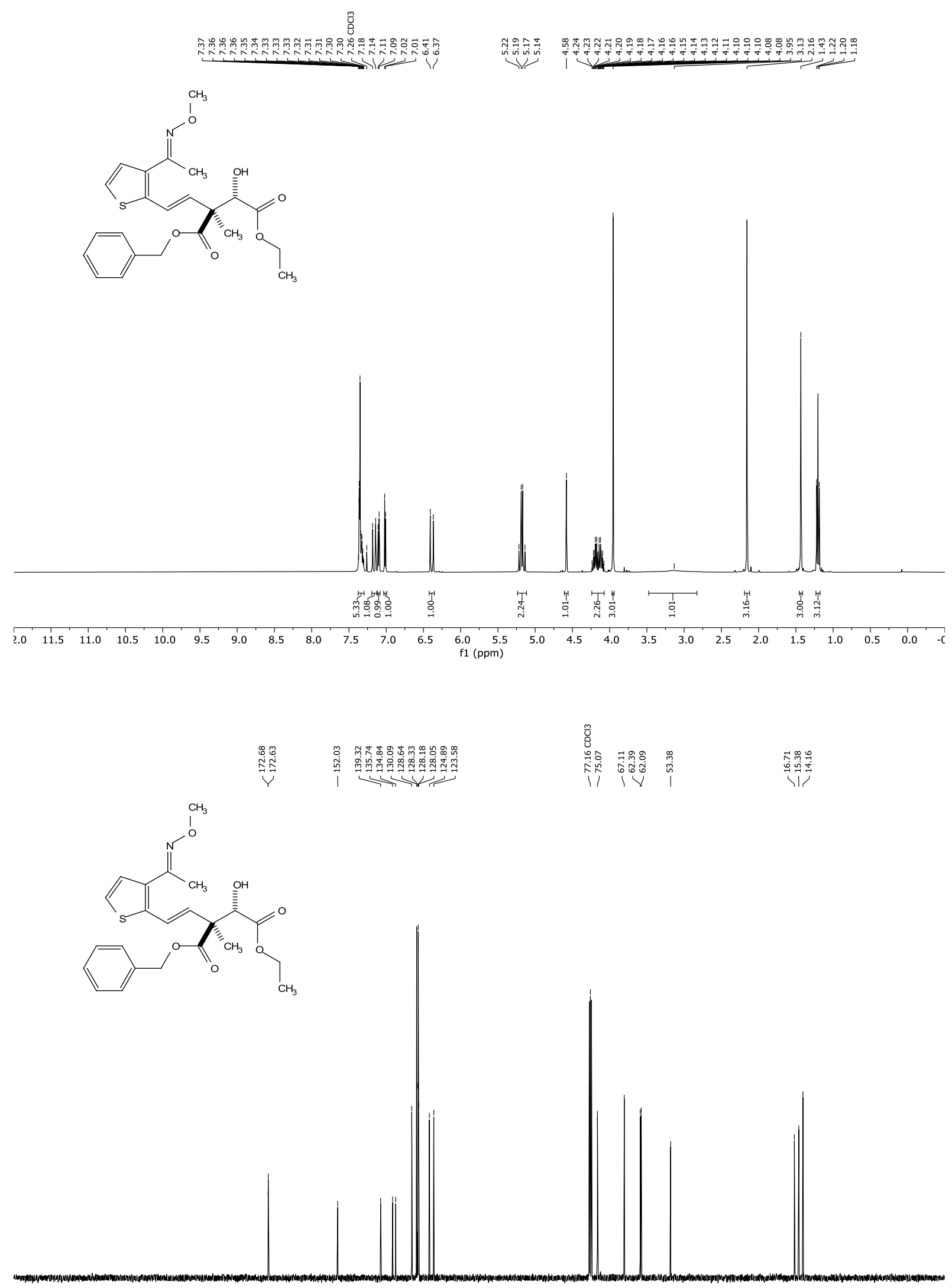

$\begin{array}{lllllllllllllllllllllllllllllllll}1 & 1 \\ 240 & 230 & 220 & 210 & 200 & 190 & 180 & 170 & 160 & 150 & 140 & 130 & 120 & 110 & 100 & 90 & 80 & 70 & 60 & 50 & 40 & 30 & 20 & 10 & 0 & -10 & -20\end{array}$ 

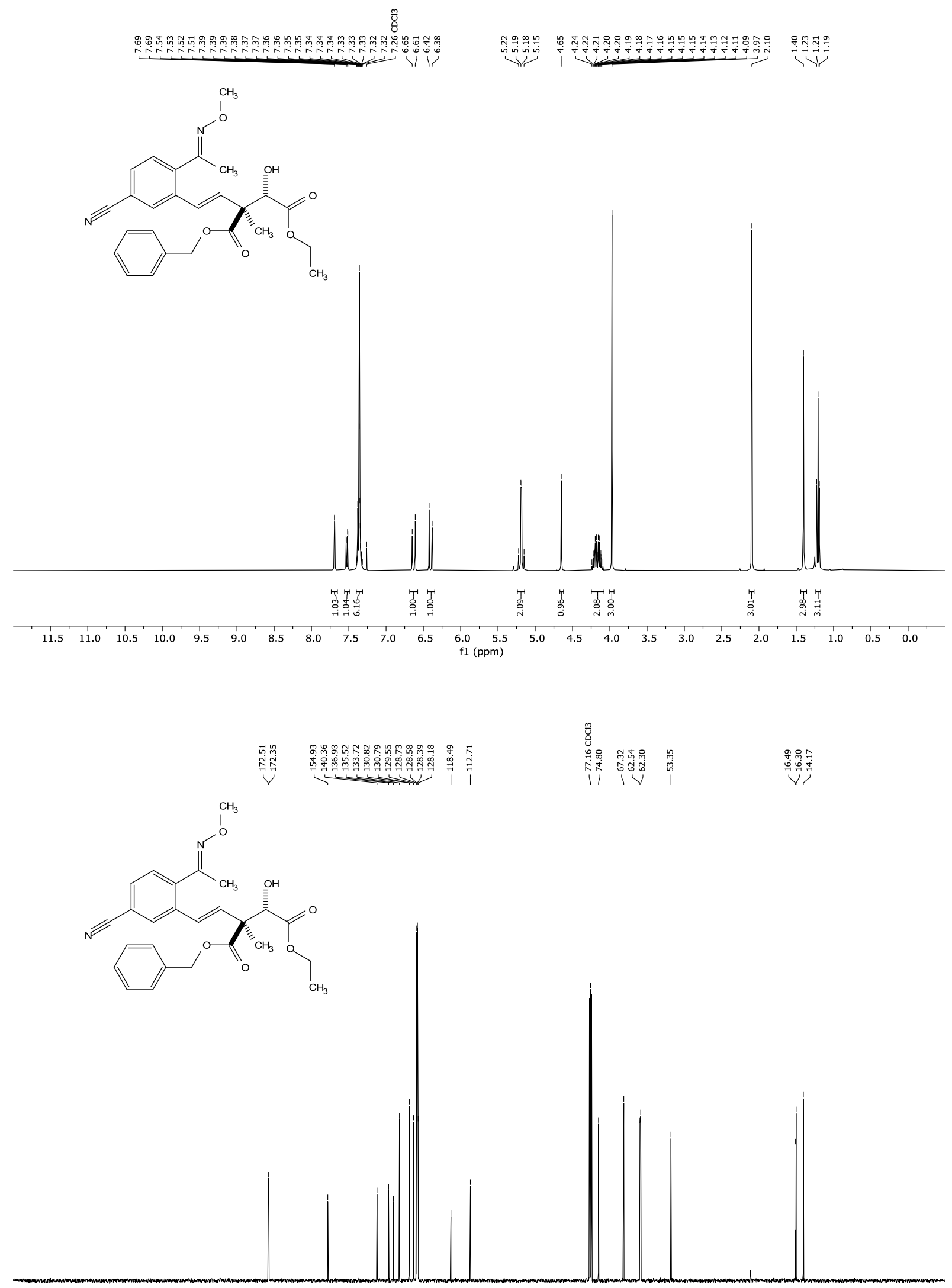

$\begin{array}{llllllllllllllllllllllllllllllll}1 & 140 & 230 & 220 & 210 & 200 & 190 & 180 & 170 & 160 & 150 & 140 & 130 & 120 & 110 & 100 & 90 & 80 & 70 & 60 & 50 & 40 & 30 & 20 & 10 & 0 & -10 & -20\end{array}$ 


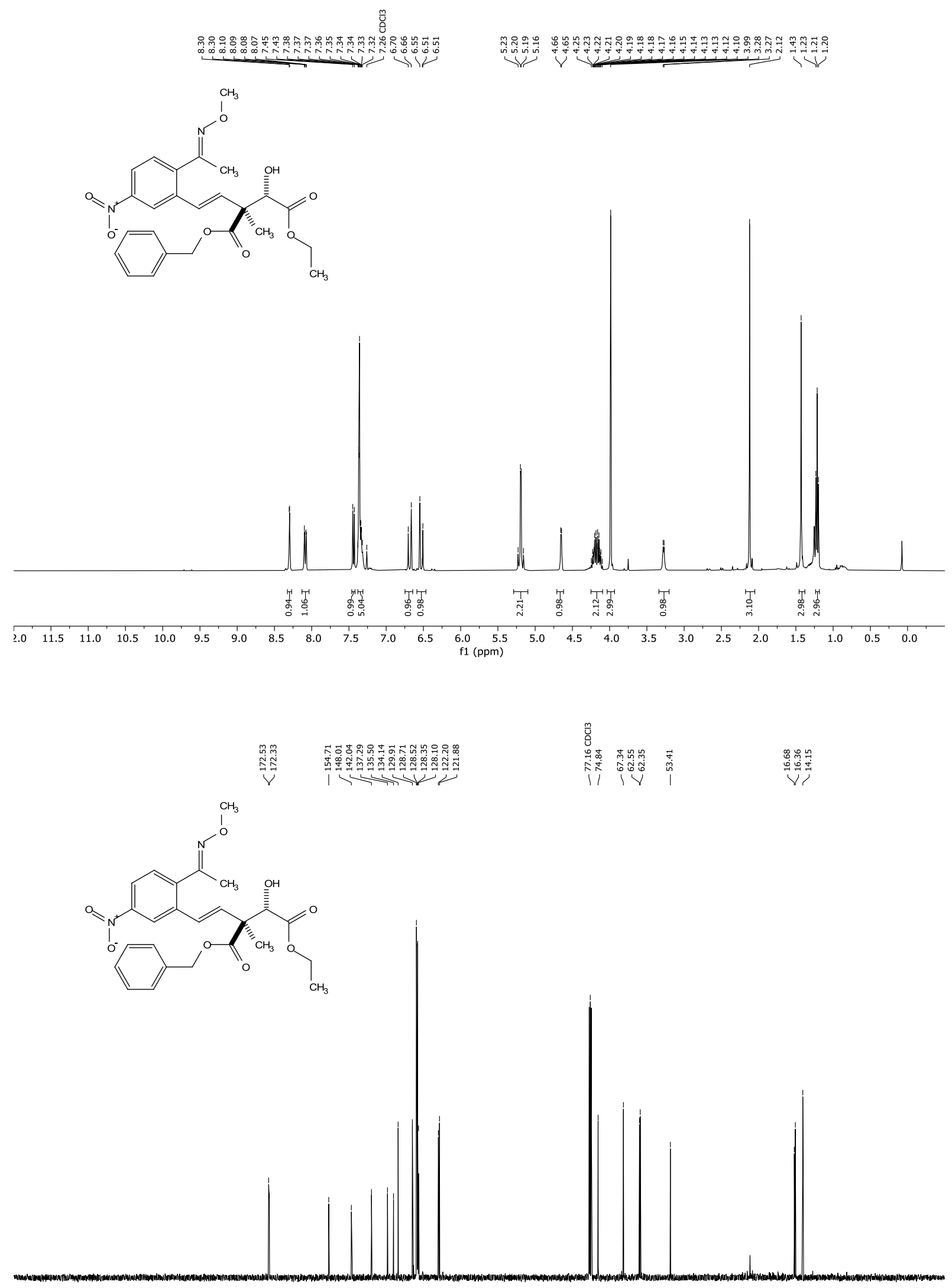

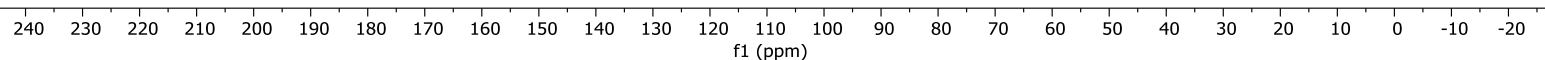




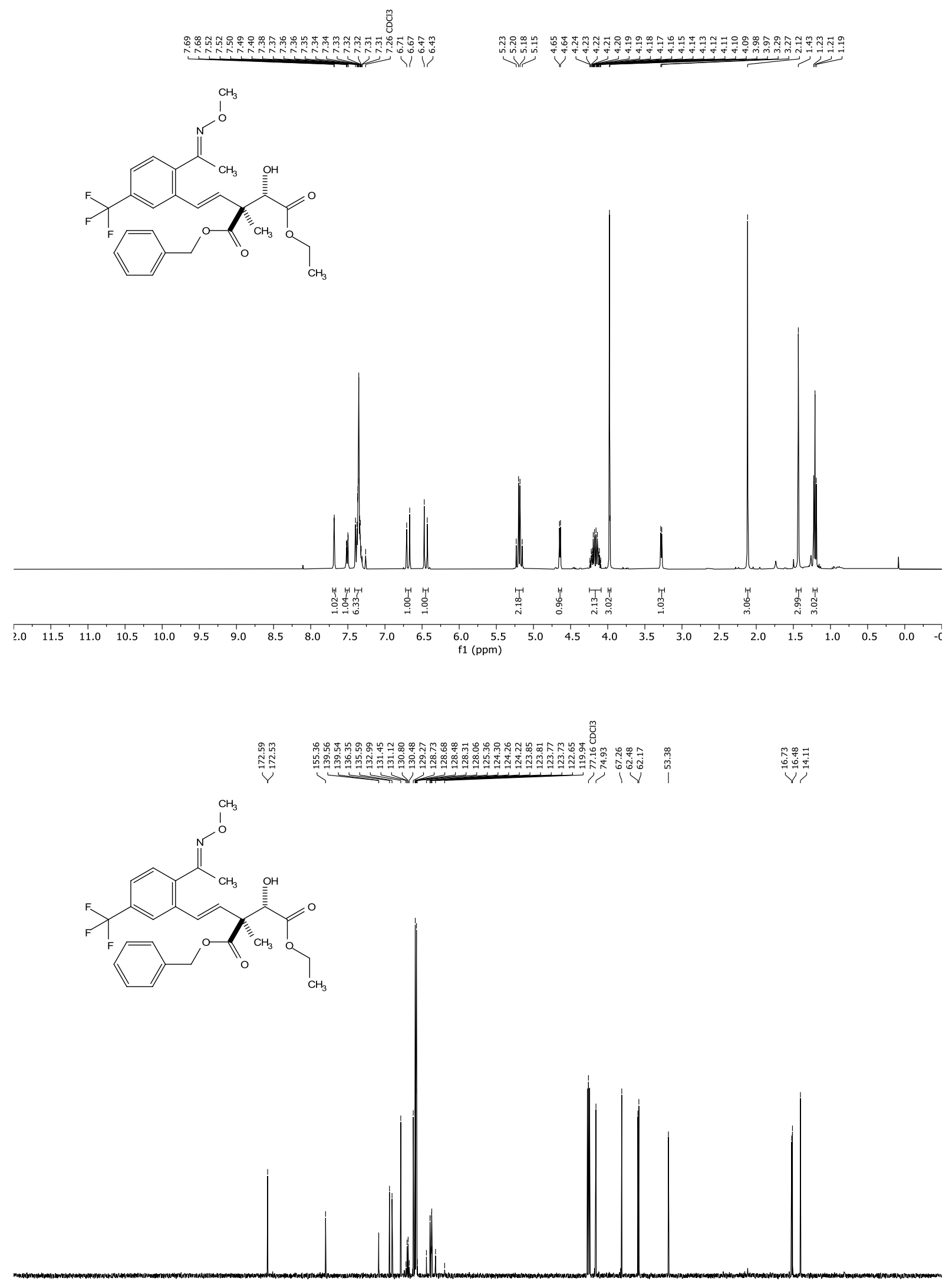

$\begin{array}{llllllllllllllllllllllllllll}1 & 240 & 230 & 220 & 210 & 200 & 190 & 180 & 170 & 160 & 150 & 140 & 130 & 120 & 110 & 100 & 90 & 80 & 70 & 60 & 50 & 40 & 30 & 20 & 10 & 0 & -10 & -20\end{array}$ 


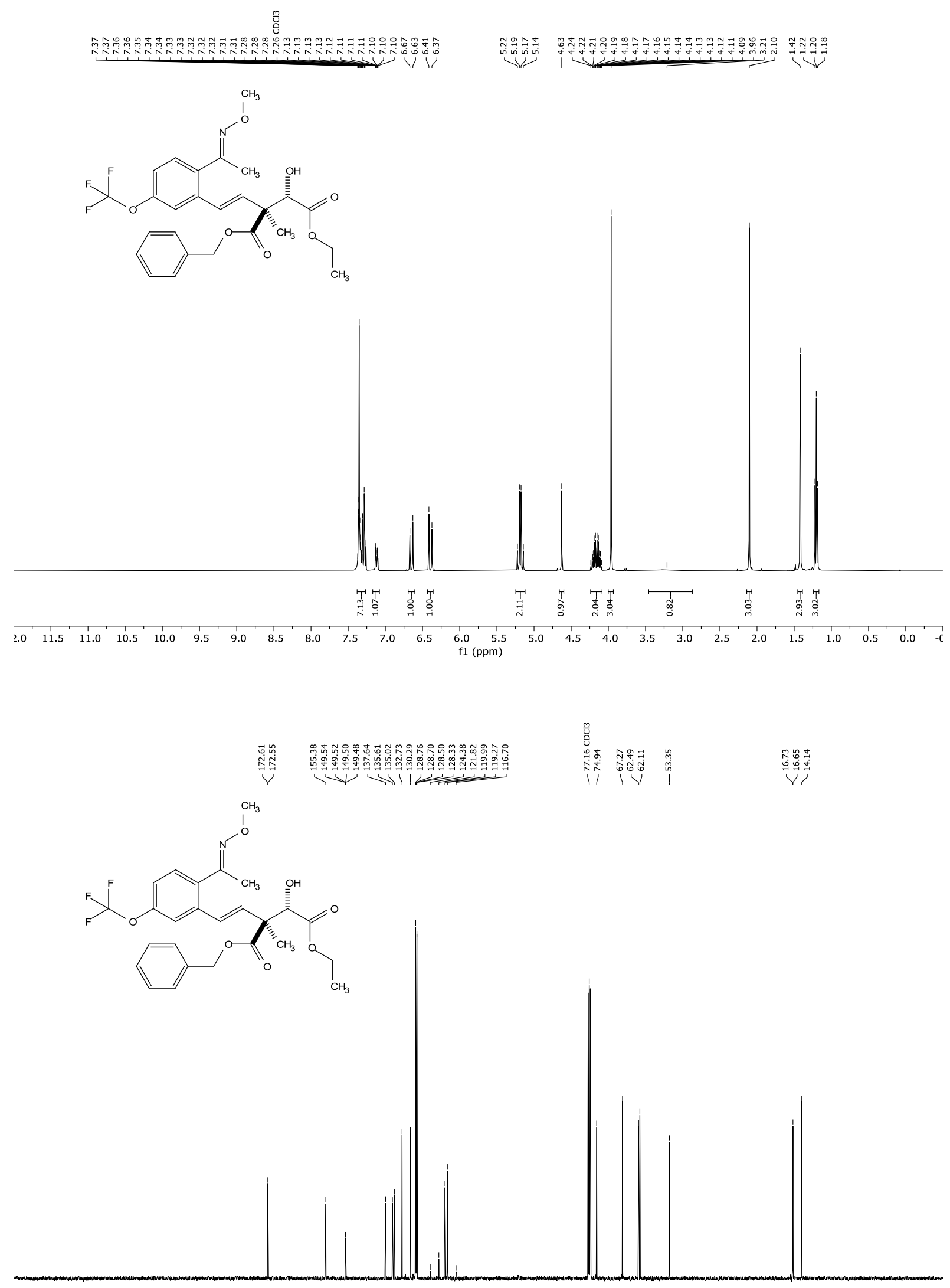

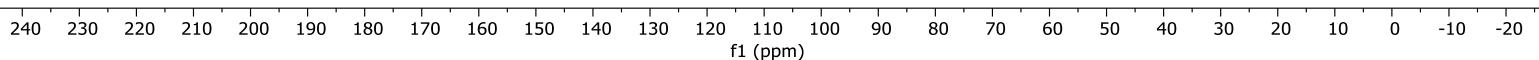




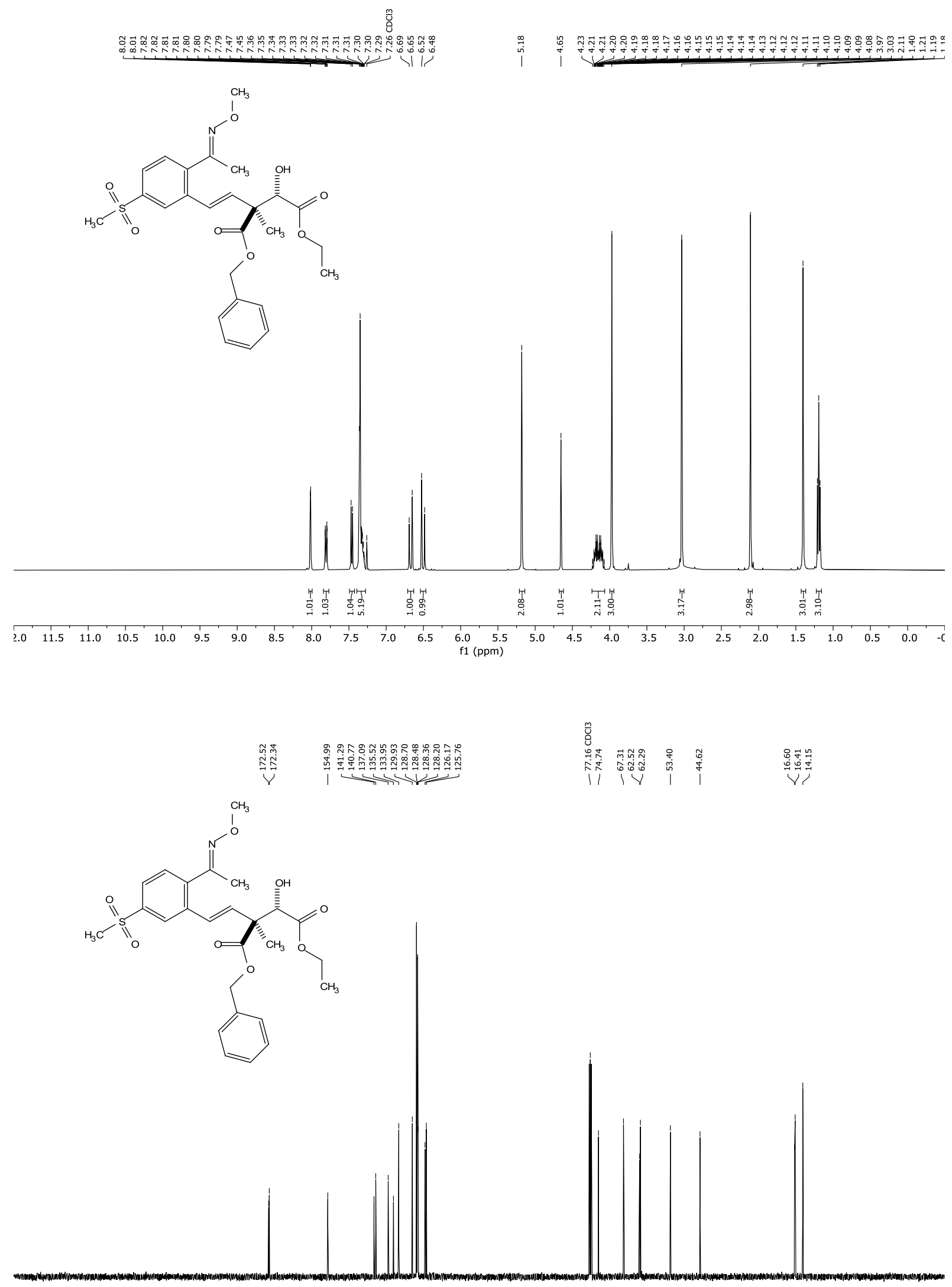

$\begin{array}{llllllllllllllllllllllllllllll}240 & 230 & 220 & 210 & 200 & 190 & 180 & 170 & 160 & 150 & 140 & 130 & 120 & 110 & 100 & 90 & 80 & 70 & 60 & 50 & 40 & 30 & 20 & 10 & 0 & -10 & -20\end{array}$ 


\section{\%}

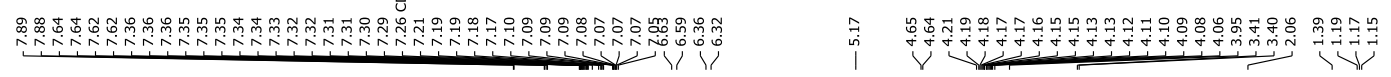
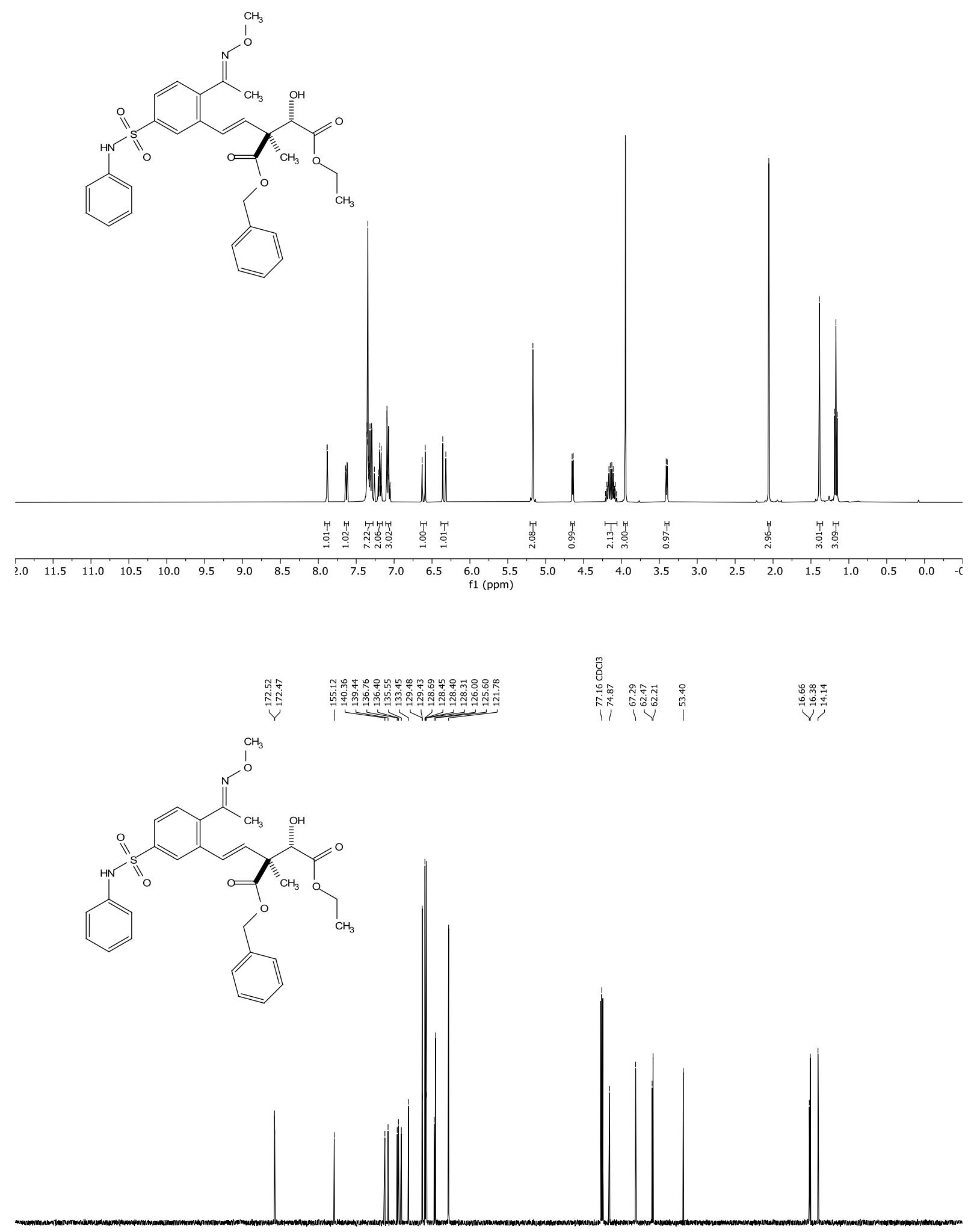

$\begin{array}{rllllllllllllllllllllllllllll}1 & 240 & 230 & 220 & 210 & 200 & 190 & 180 & 170 & 160 & 150 & 140 & 130 & 120 & 110 & 100 & 90 & 80 & 70 & 60 & 50 & 40 & 30 & 20 & 10 & 0 & -10 & -20\end{array}$ 

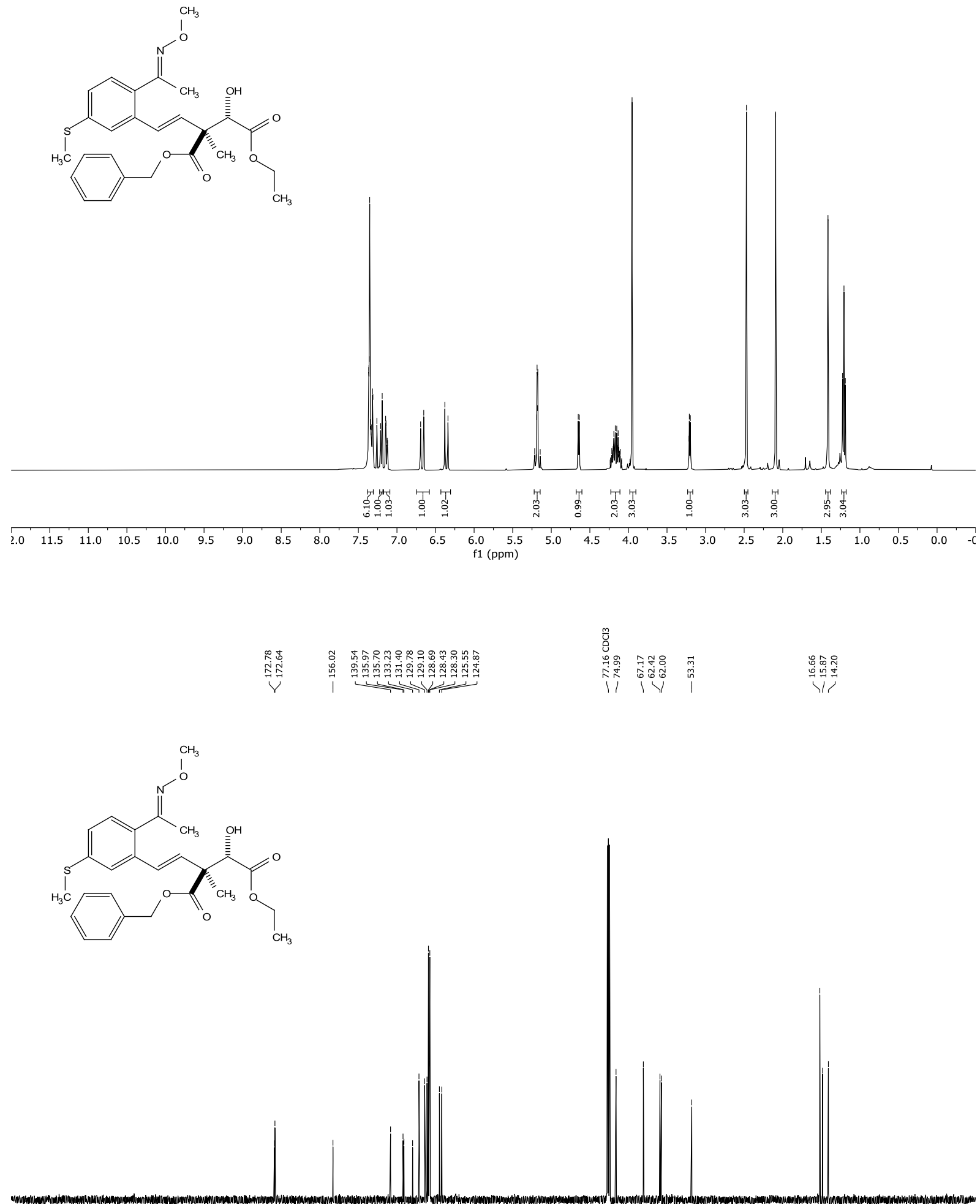

$\begin{array}{llllllllllllllllllllllllllllllll}240 & 230 & 220 & 210 & 200 & 190 & 180 & 170 & 160 & 150 & 140 & 130 & 120 & 110 & 100 & 90 & 80 & 70 & 60 & 50 & 40 & 30 & 20 & 10 & 0 & -10 & -20\end{array}$ 


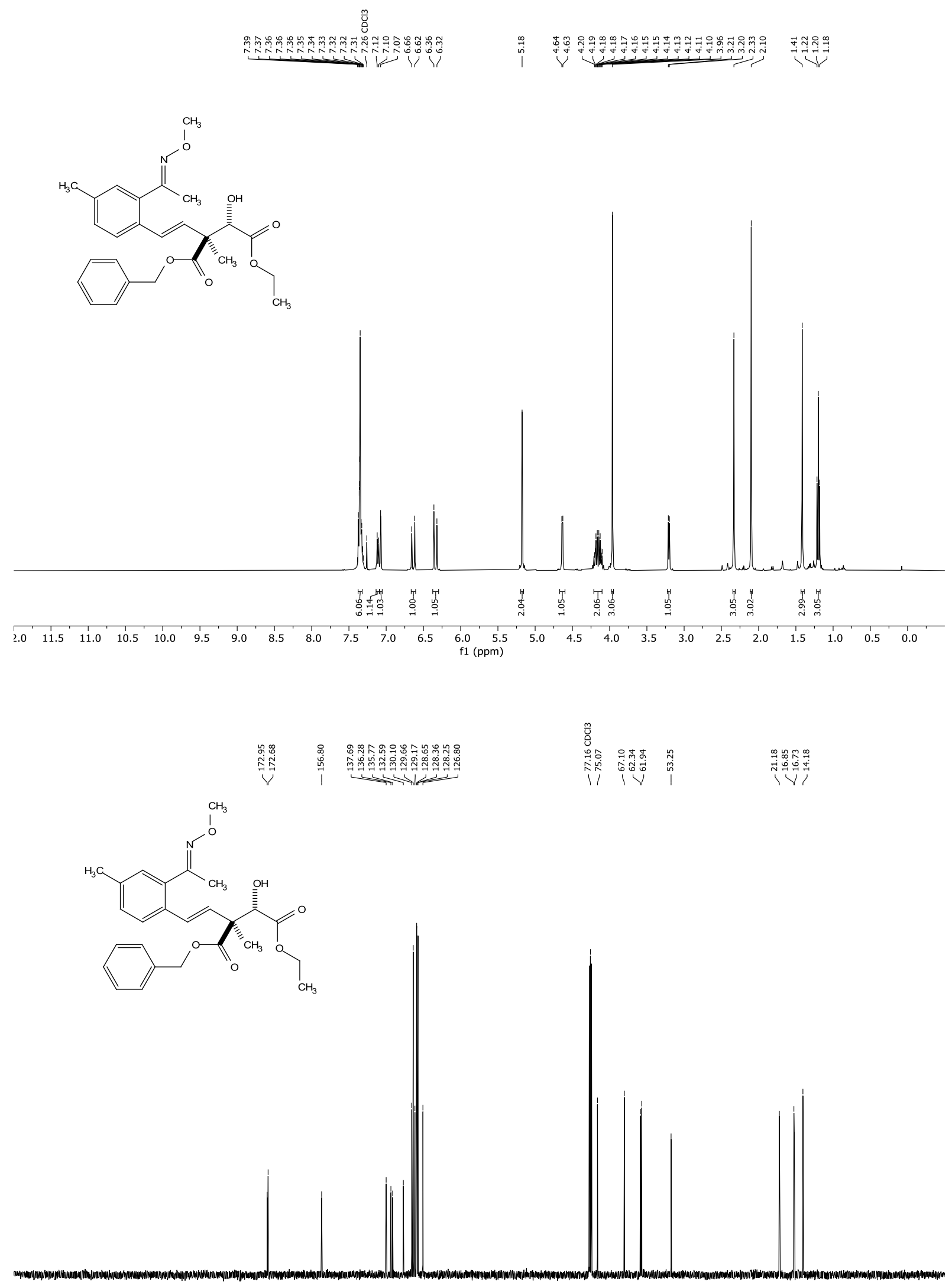

$\begin{array}{lllllllllllllllllllllllllllllllll}1 & 1 \\ 240 & 230 & 220 & 210 & 200 & 190 & 180 & 170 & 160 & 150 & 140 & 130 & 120 & 110 & 100 & 90 & 80 & 70 & 60 & 50 & 40 & 30 & 20 & 10 & 0 & -10 & -20\end{array}$ 


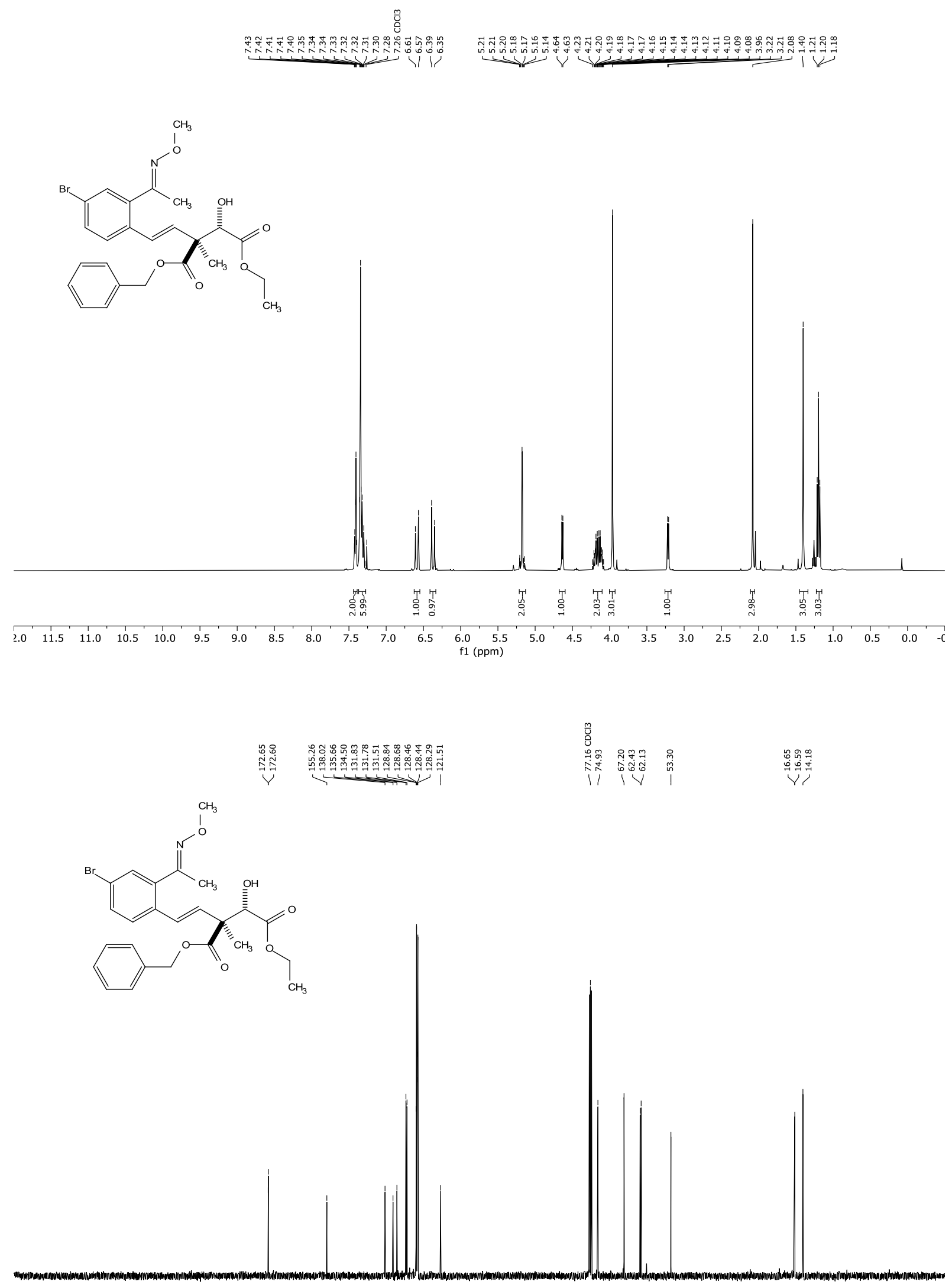

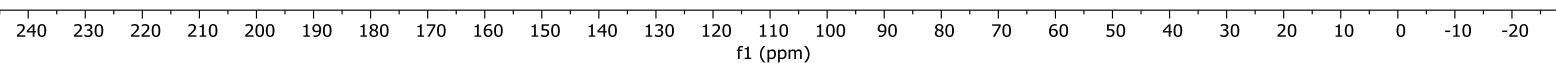



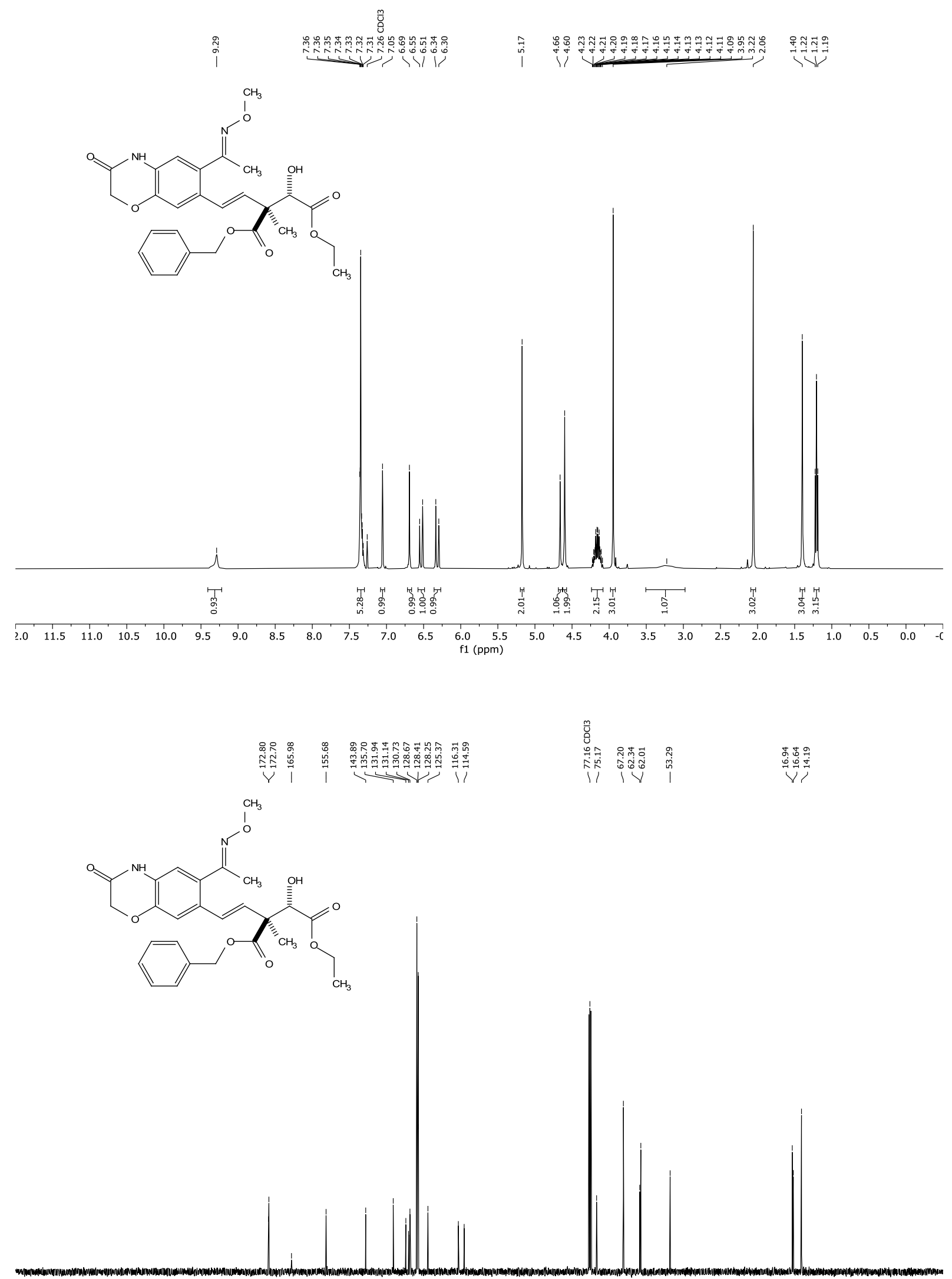

$\begin{array}{lllllllllllllllllllllllllllll}1 & 1 \\ 240 & 230 & 210 & 200 & 190 & 180 & 170 & 160 & 150 & 140 & 130 & 120 & 110 & 100 & 90 & 80 & 70 & 60 & 50 & 40 & 30 & 20 & 10 & 0 & -10 & -20\end{array}$ 


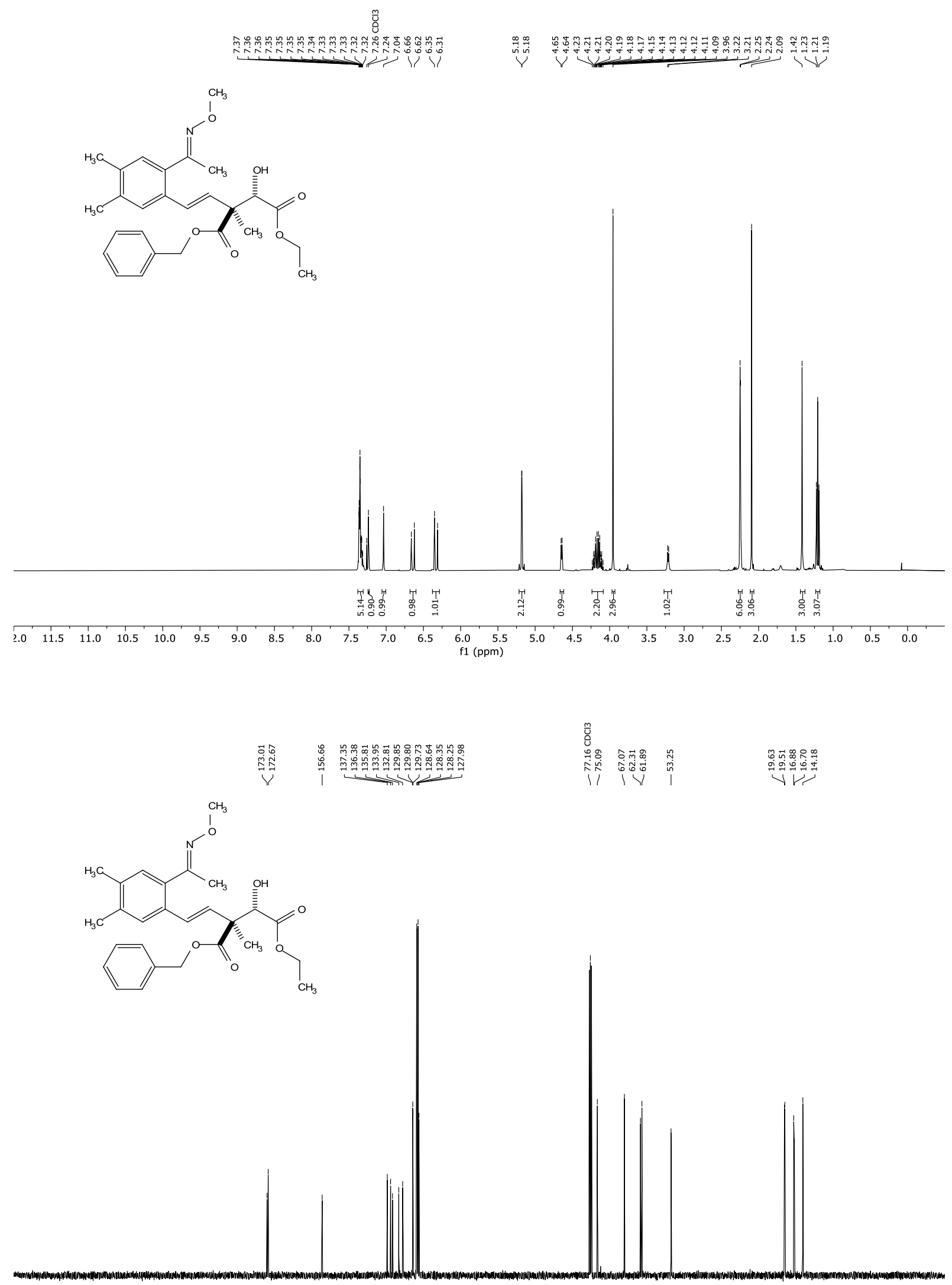

$\begin{array}{llllllllllllllllllllllllllllll}1 & 240 & 230 & 220 & 210 & 200 & 190 & 180 & 170 & 160 & 150 & 140 & 130 & 120 & 110 & 100 & 90 & 80 & 70 & 60 & 50 & 40 & 30 & 20 & 10 & 0 & -10 & -20\end{array}$ 

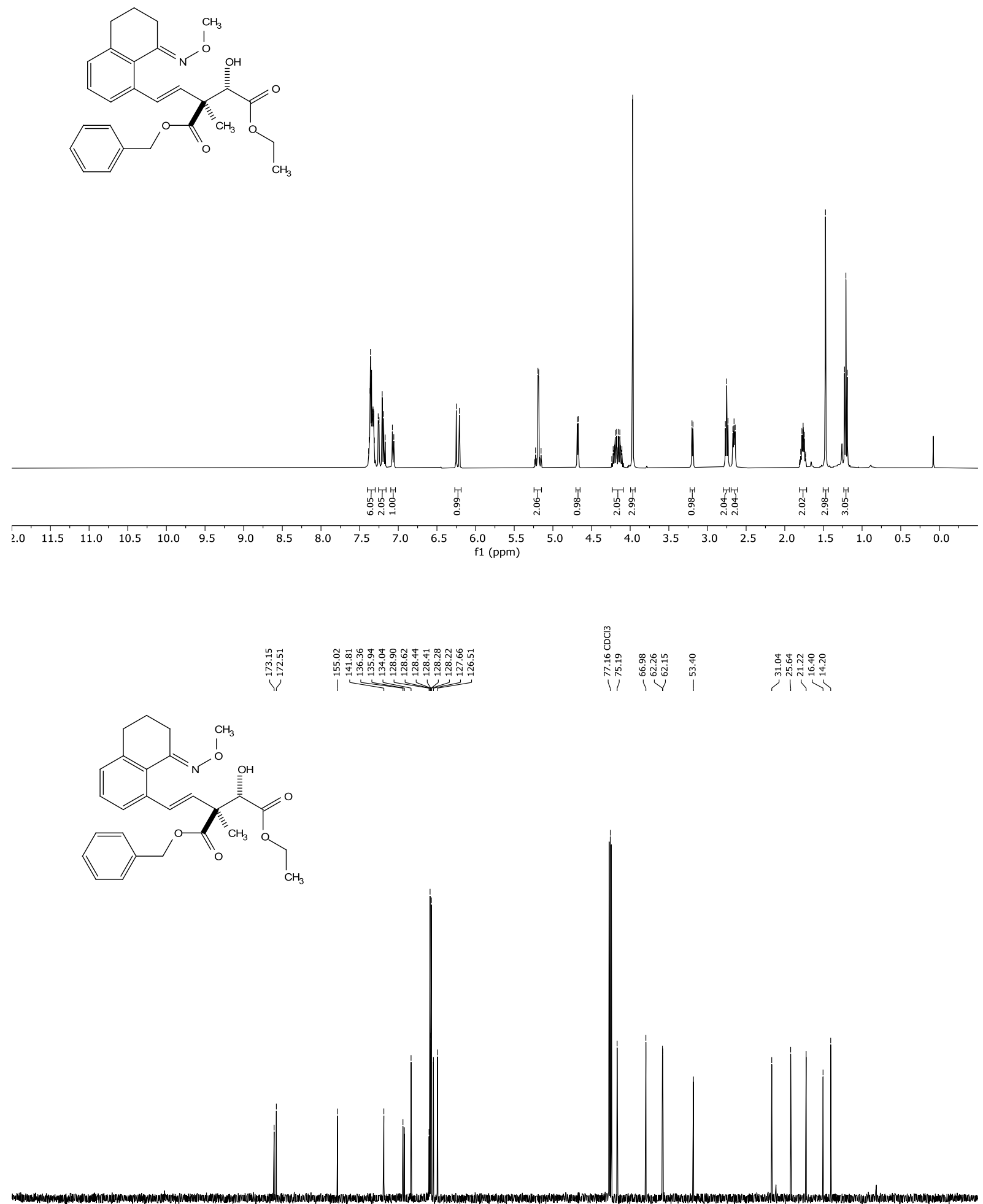

$\begin{array}{llllllllllllllllllllllllllllll}240 & 230 & 220 & 210 & 200 & 190 & 180 & 170 & 160 & 150 & 140 & 130 & 120 & 110 & 100 & 90 & 80 & 70 & 60 & 50 & 40 & 30 & 20 & 10 & 0 & -10 & -20\end{array}$ 


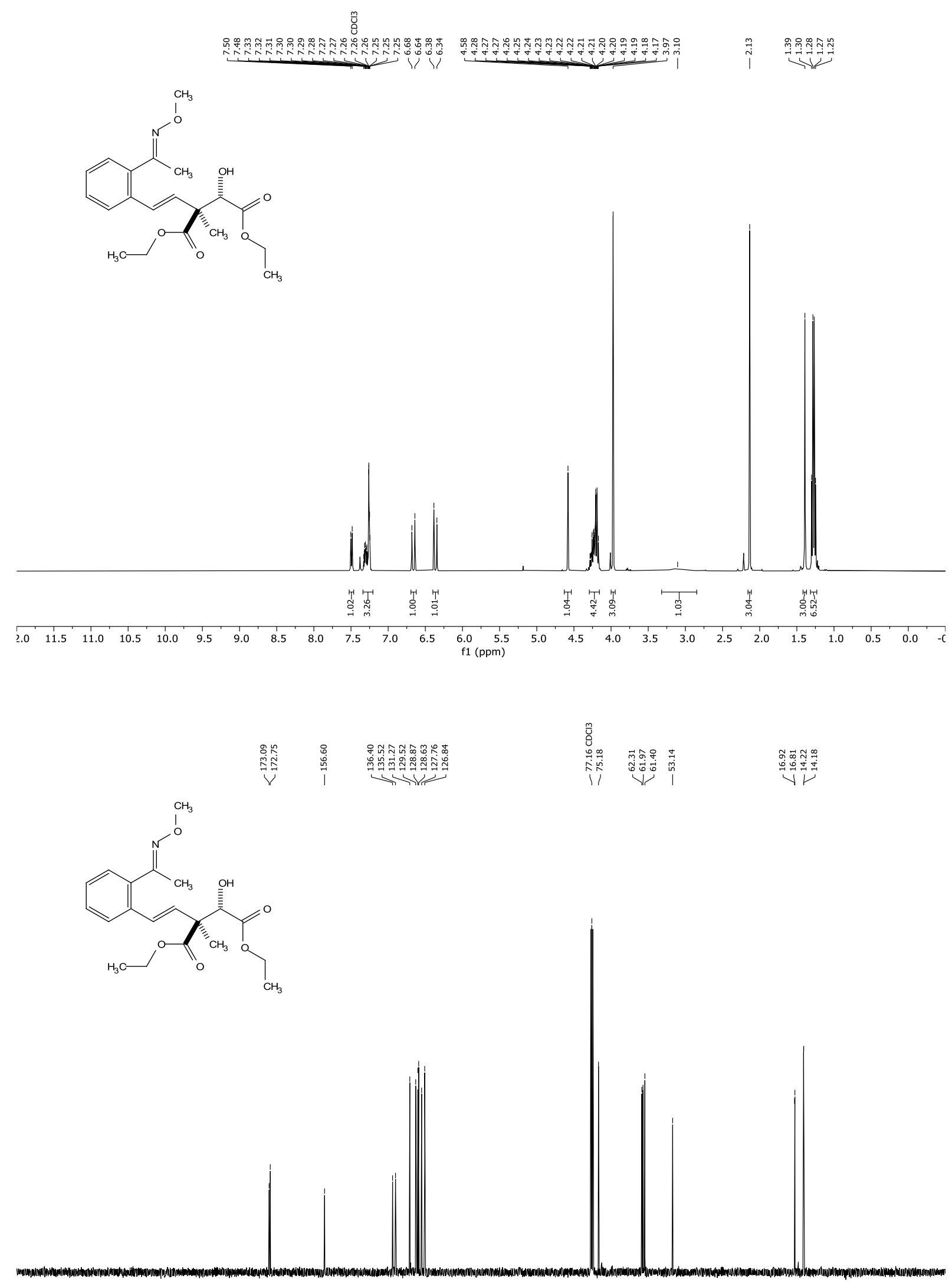

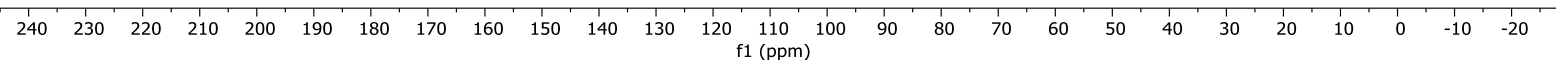




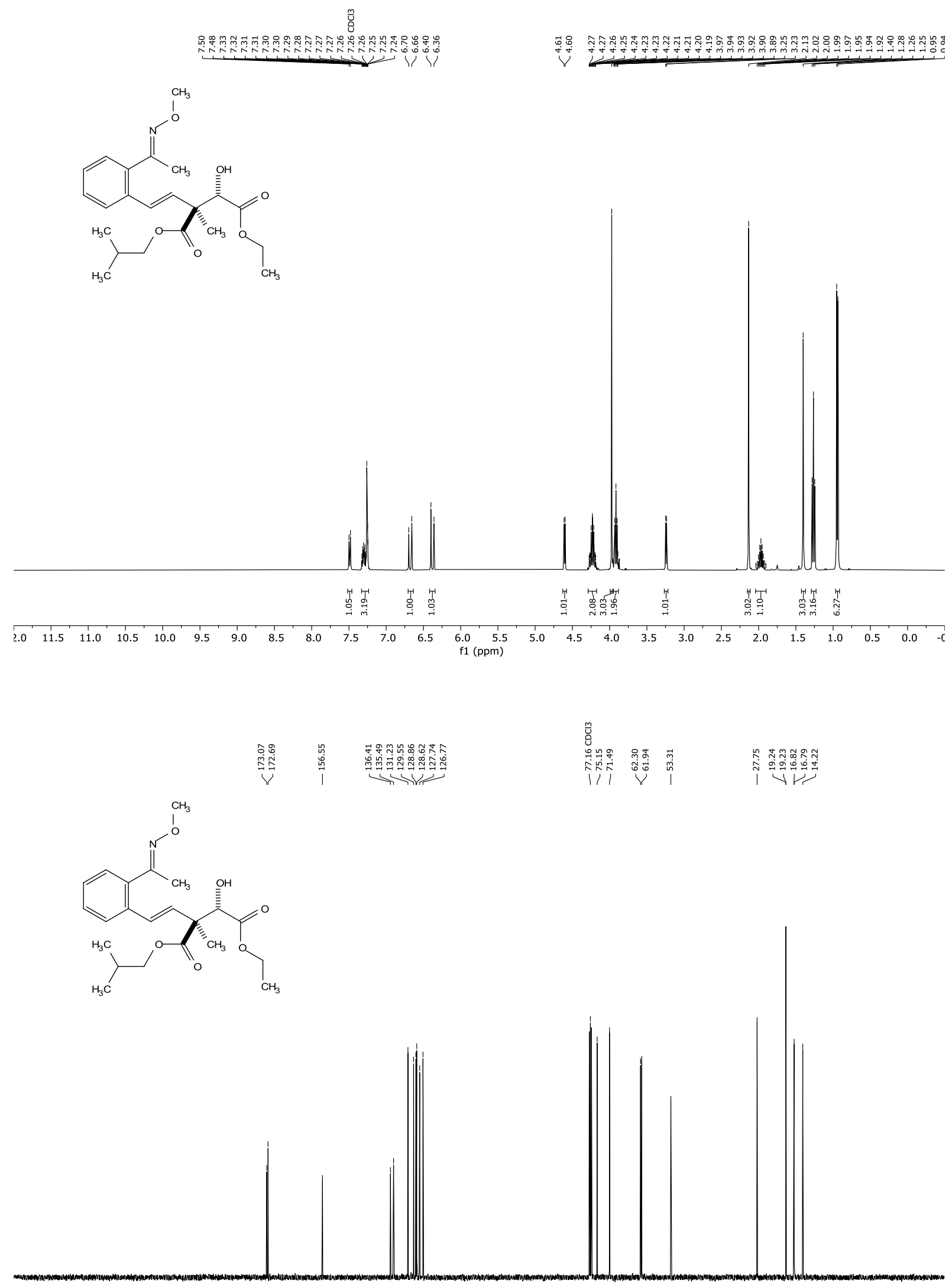

$\begin{array}{llllllllllllllllllllllllllllllll}1 & 1 \\ 240 & 230 & 220 & 210 & 200 & 190 & 180 & 170 & 160 & 150 & 140 & 130 & 120 & 110 & 100 & 90 & 80 & 70 & 60 & 50 & 40 & 30 & 20 & 10 & 0 & -10 & -20\end{array}$ 


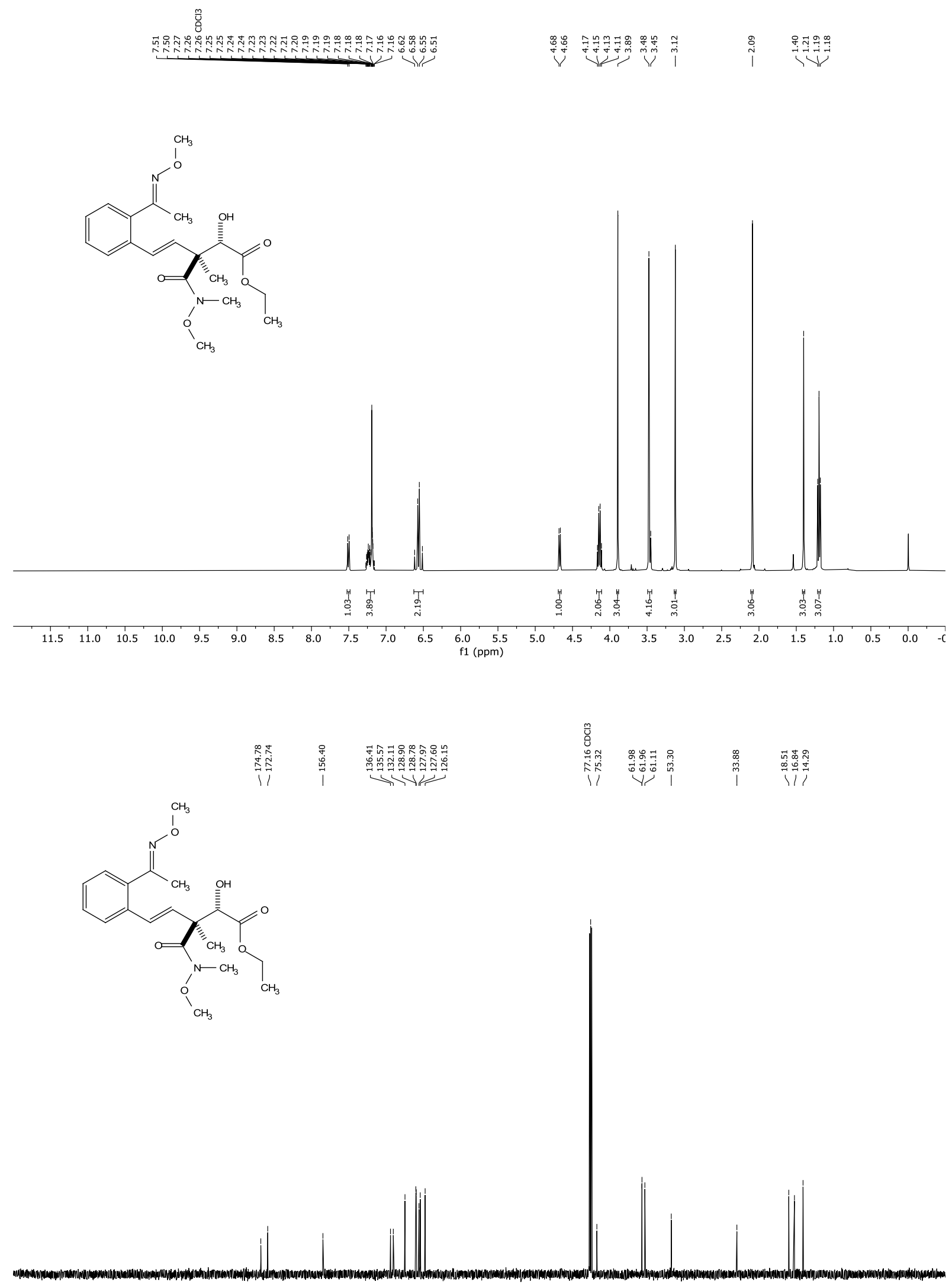

$\begin{array}{llllllllllllllllllllllllllllllll}240 & 230 & 220 & 210 & 200 & 190 & 180 & 170 & 160 & 150 & 140 & 130 & 120 & 110 & 100 & 90 & 80 & 70 & 60 & 50 & 40 & 30 & 20 & 10 & 0 & -10 & -20\end{array}$ 


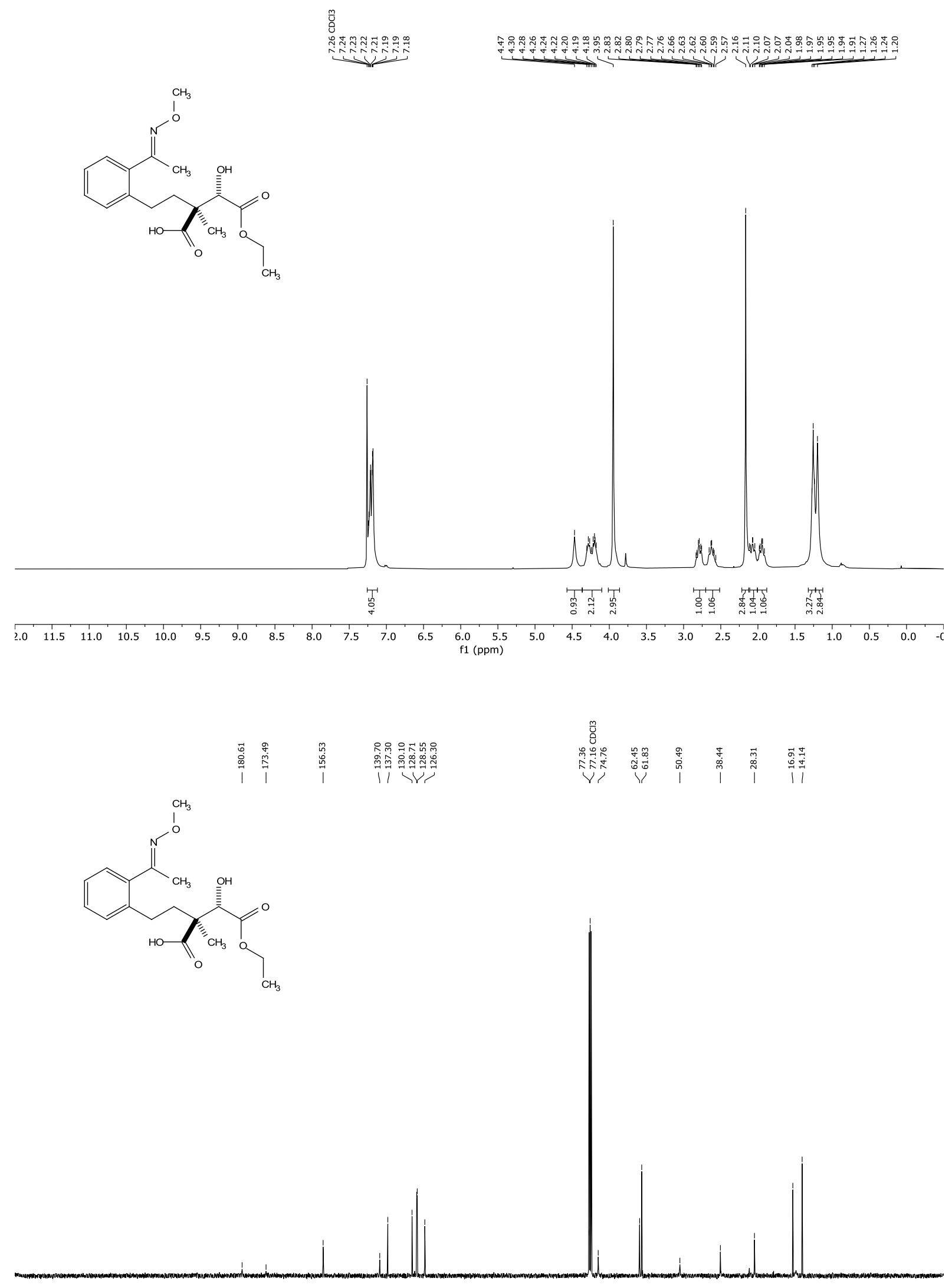

$\begin{array}{lllllllllllllllllllllllllll}140 & 230 & 220 & 210 & 200 & 190 & 180 & 170 & 160 & 150 & 140 & 130 & 120 & 110 & 100 & 90 & 80 & 70 & 60 & 50 & 40 & 30 & 20 & 10 & 0 & -10 & -20\end{array}$ 


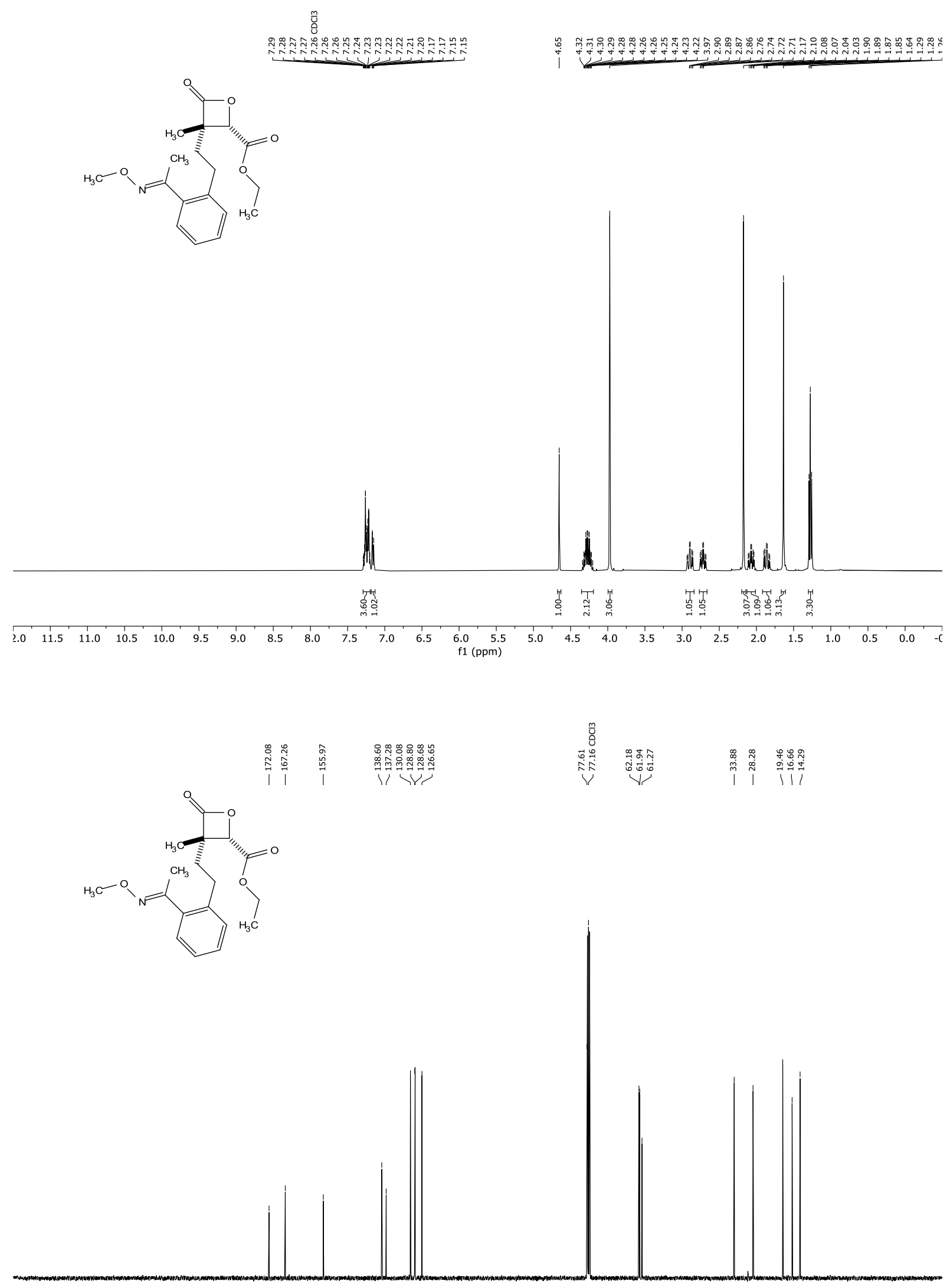

$\begin{array}{llllllllllllllllllllllllllll}1 & 240 & 230 & 220 & 210 & 200 & 190 & 180 & 170 & 160 & 150 & 140 & 130 & 120 & 110 & 100 & 90 & 80 & 70 & 60 & 50 & 40 & 30 & 20 & 10 & 0 & -10 & -20\end{array}$ 

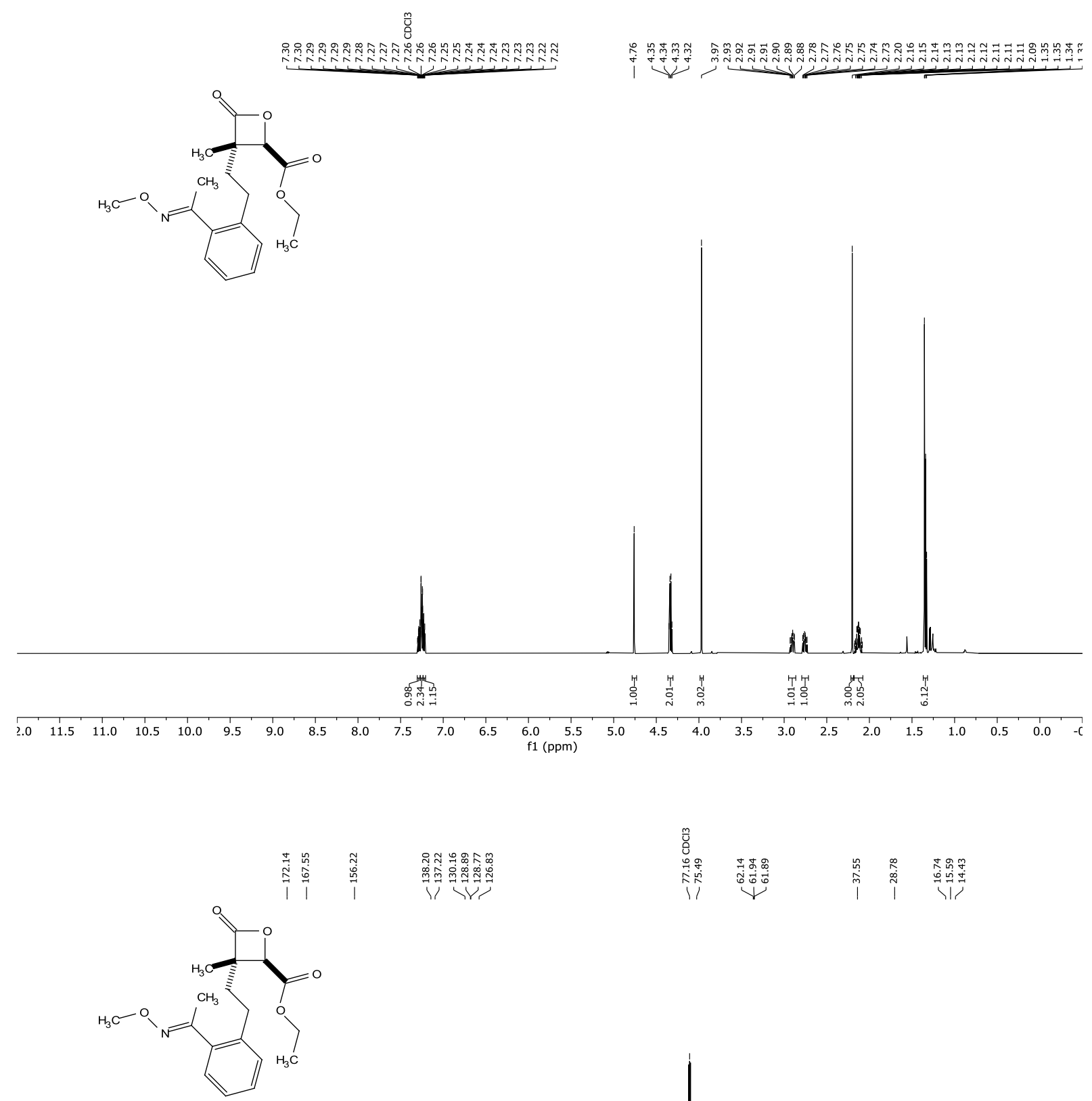

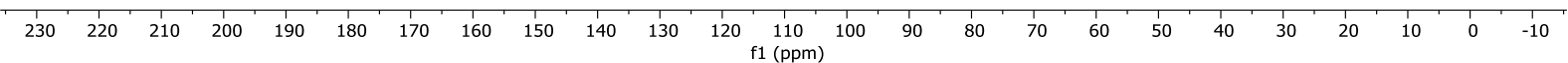




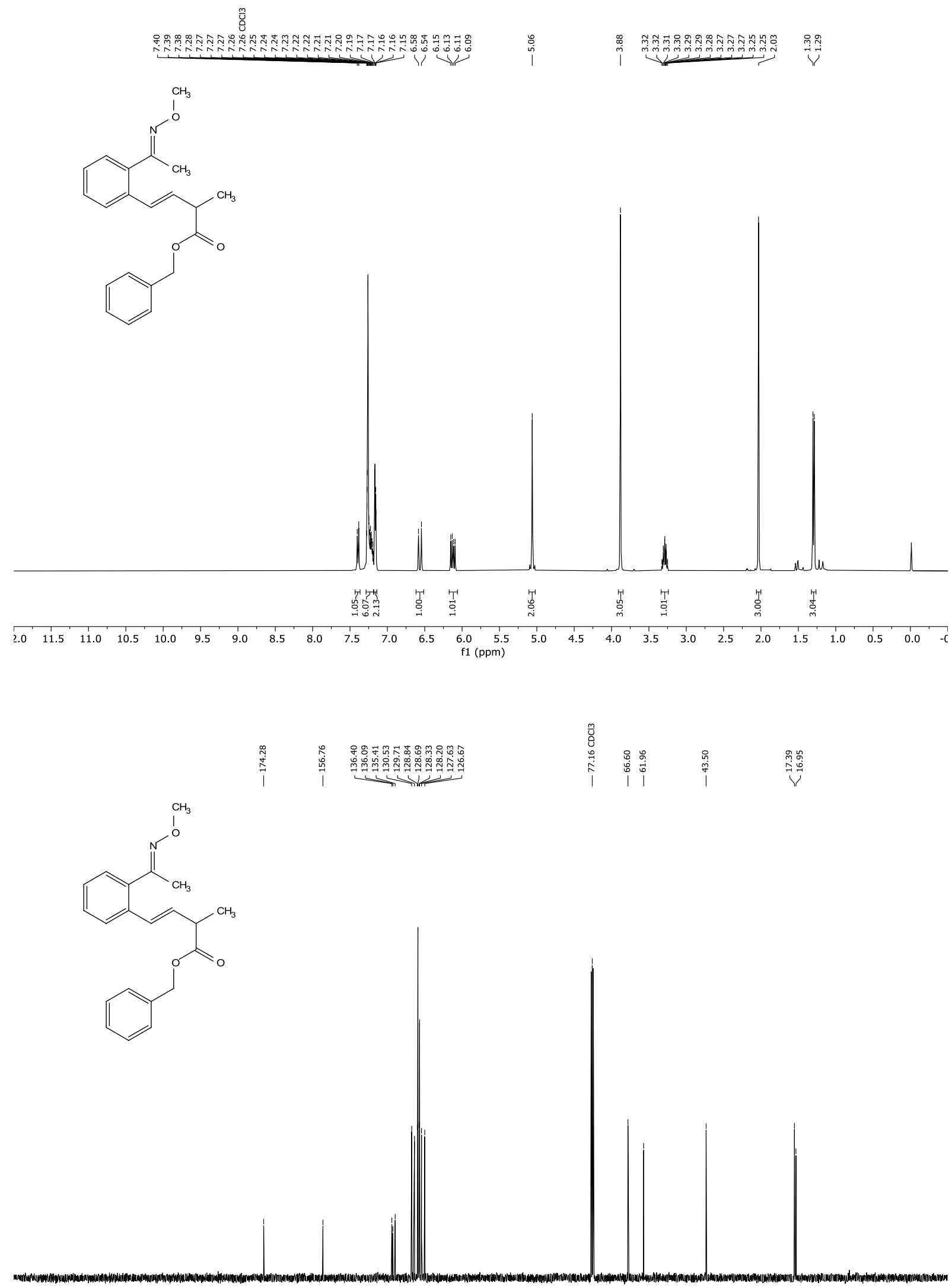

$\begin{array}{lllllllllllllllllllllllllllll}1 & 240 & 230 & 220 & 210 & 200 & 190 & 180 & 170 & 160 & 150 & 140 & 130 & 120 & 110 & 100 & 90 & 80 & 70 & 60 & 50 & 40 & 30 & 20 & 10 & 0 & -10 & -20\end{array}$ 

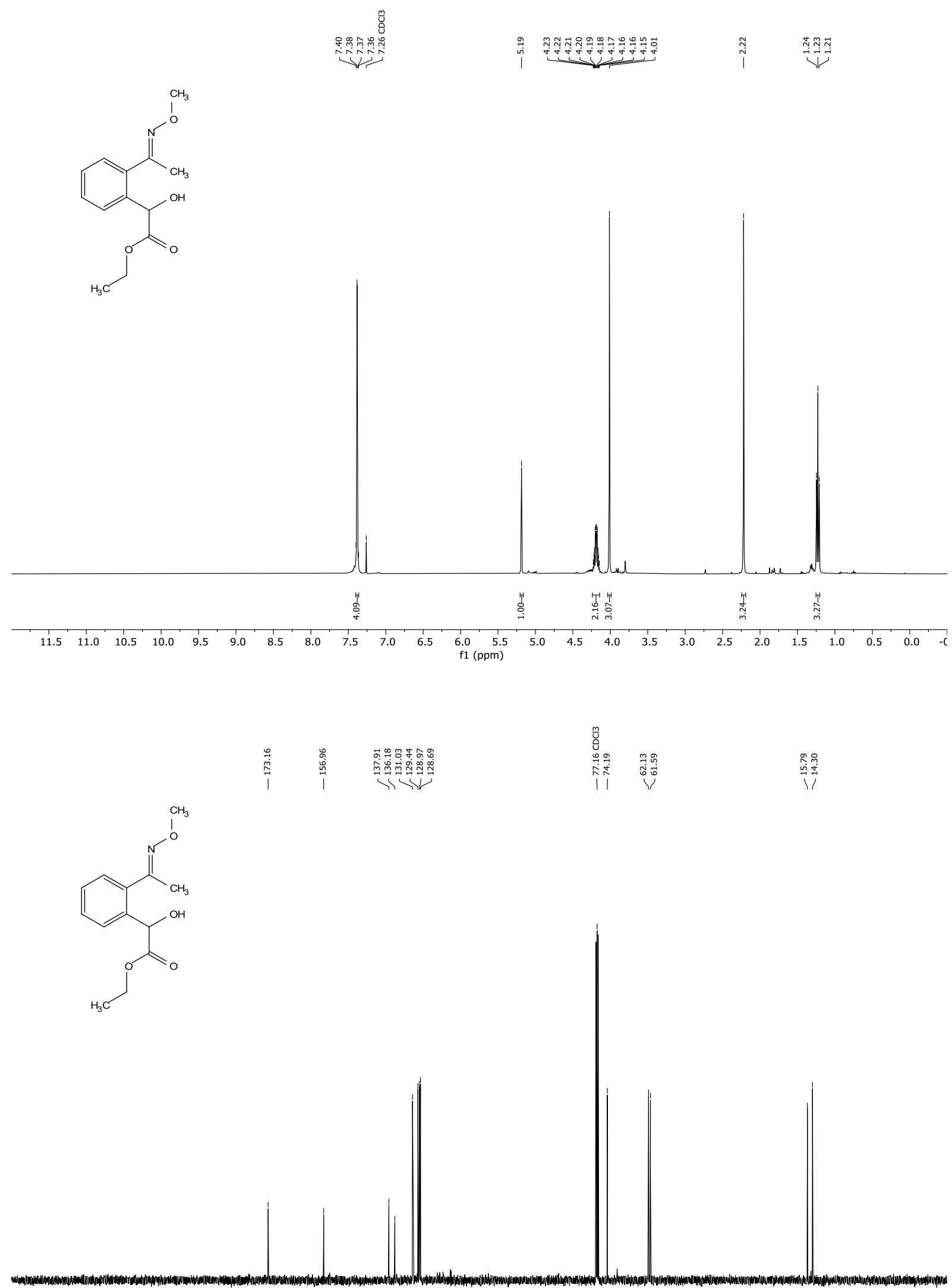

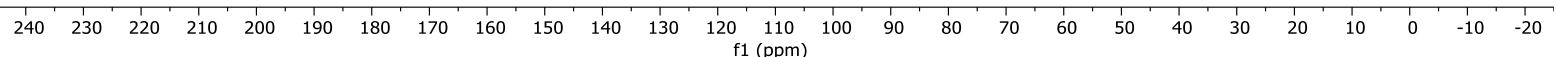



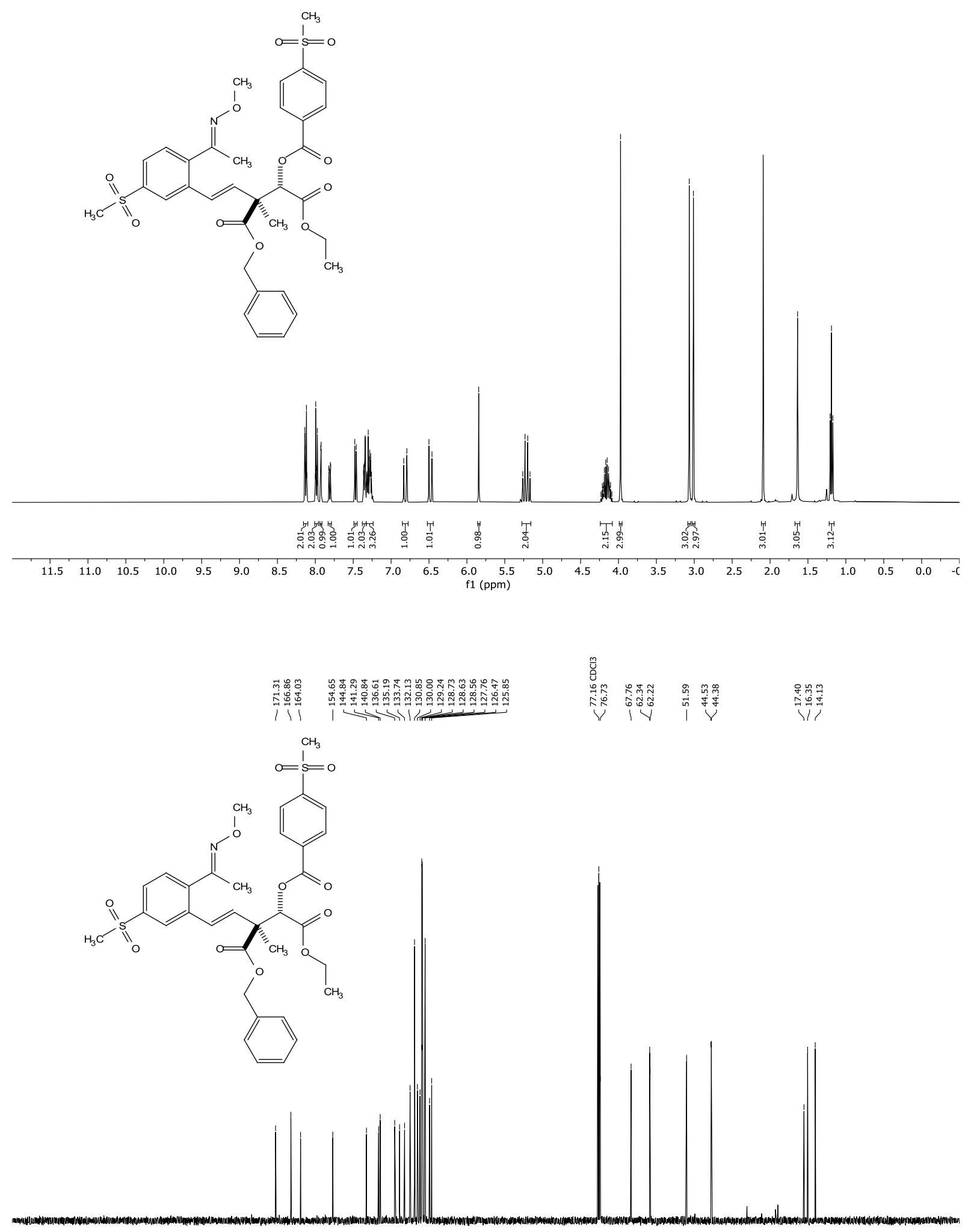

$\begin{array}{llllllllllllllllllllllllllllll}1 & 1 \\ 240 & 230 & 220 & 210 & 200 & 190 & 180 & 170 & 160 & 150 & 140 & 130 & 120 & 110 & 100 & 90 & 80 & 70 & 60 & 50 & 40 & 30 & 20 & 10 & 0 & -10 & -20\end{array}$ 

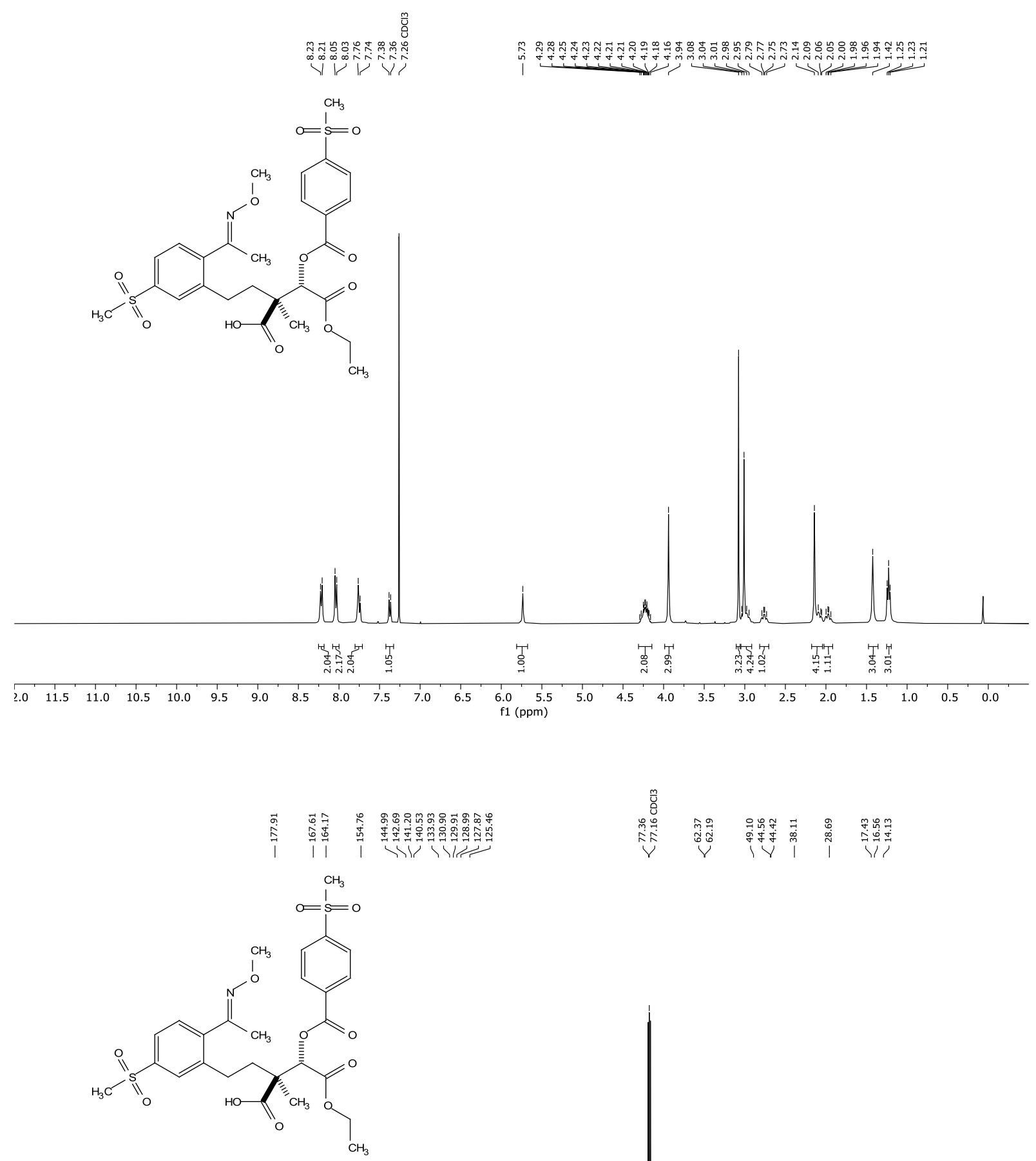